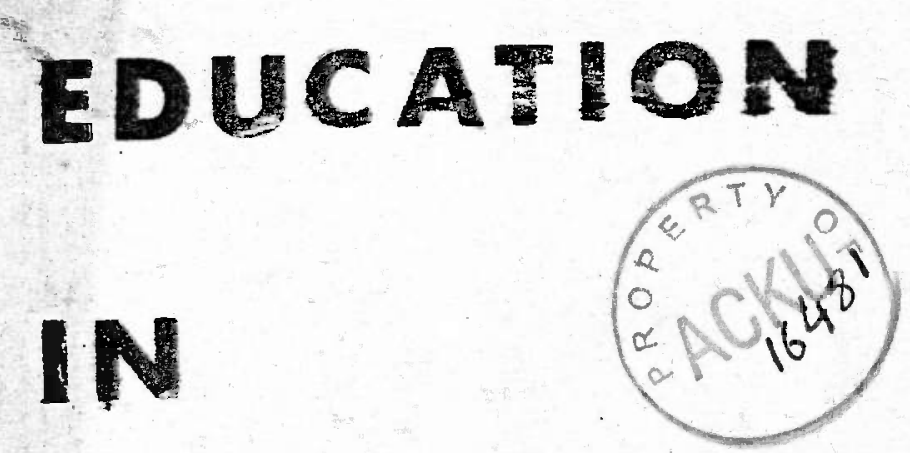

\title{
AFGHANISTAN
}

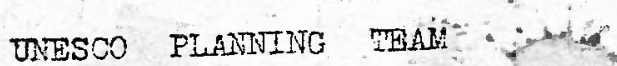
UINISTRY OF EDUCATION $\mathrm{KABCL}$

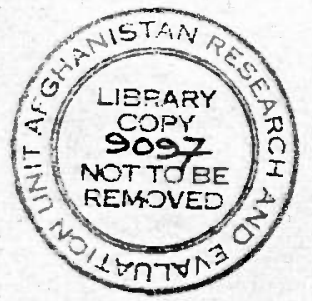




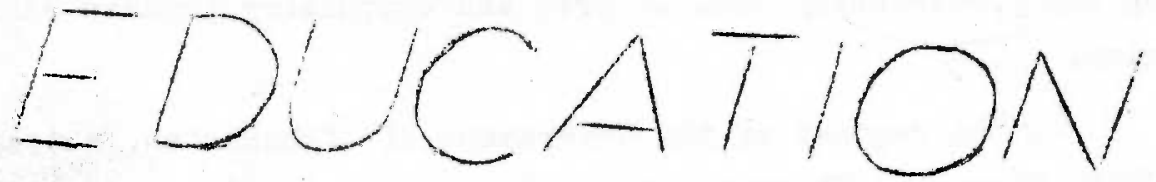

IN
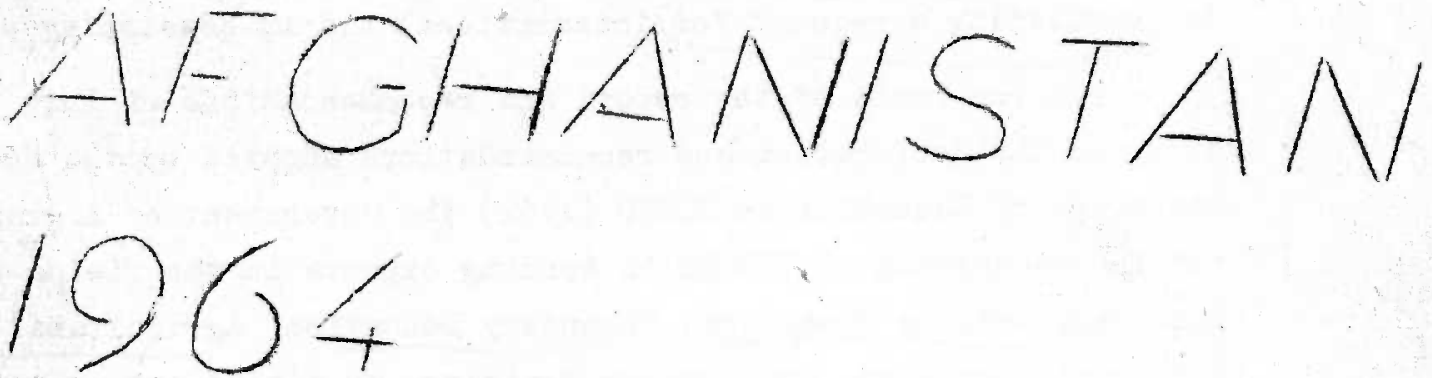

$+$

UNESCO. PLANNING TEAM MINISTRY OF EDUCATION December 1964

KABUI

The compilation of these date and their presentation are the responsibilety of the members of the UNESCO Planning Team. 
The Regional lleeting of Representatives of Asian Member States on Primary and Compulsory Education (KARACHI 1960) adopted a recommendation concerning the provision by 1980 of free and compulsory primary education in the region.

At the request of the Government of Afghanistan, a team of consultants (UNESCO Mducation Planning Iission) was sent to Afghanistan early in 1962 in order to make a general survey of education, to make recommendations concerning the overall planning of education within the context of national economic and social development, and especially to advise and assist the Government in formulating a request for international aid in developing education.

On the basis of the report and recommendations of this planning mission and the declaration and recommandations adopted by the lleeting of Asian Ministers of Education in TOKYO (1962) the Government of Afghanistan requested the assistance of UNESCO in sending experts in the fields of Educatianal Planning, Primary Education, Secondary Education, Agricultural Education, Technical Education, and Teacher Training, to advise and assist the Government on improvements in education and the preparation of long term plans.

Previsions for educationel development are included in the "Second Five Year Plan" (1962-1967), formulated by the Government for the develogment of the national economy.

The objectives of the Planning Team are:

a) Preparation of short and long term plans for educational expansion at all levels:

This requires the preparation of sound statistical data relating to the quantitative and qualitative appraisal of the present system and the future needs and possibilities in relation to social and economic progress.

b) The training of Afghan personnel in educational planning.

The members of the Planning Team associated with these studies are as follows :

Ir. H. MUNIER (Fr.) - Team Leader and Expert in Educational Planning; III. R.A. DICKIE (N-Z.) - Expert in Teacher Training;

Dr. S.IAGERSTEDT (Sweden) - Expert in Secondary Education;

Dr. S., LOTHI (U.A.R.) - Expert in Primary Education;

Dr. S. NAKATA (Japan) - Expert in Agricultural Education;

Dr. A. THURiver (Austria) - Expert in Technical Education. 


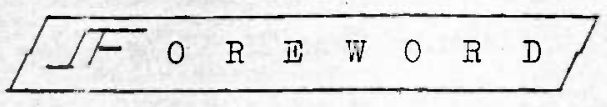

This statistical document is the product of surveys and exicuies undertaken by the UIJSCO Planning Team during the period extending from July to November 1964. It is designed to present, in an appropriate order such basic data as are cvailable at the prosent time with a wor thej use for educational planning in Afghanistan.

The main sources of the data incorporated in this document are:

1 - The returns of the survey carried out in April each yeer by the Statistical Department of the Bureau of Planning;

2 - A survey designed by the UNESCO Plaming Team covering ali achools in KABUL City and a sampling of provincial schools. For this last, an adequate probability sample was possible only for KABUI. Frovince.

All ther sources are cited in the tert.

The principal symbols used are the following:

- Magnitude nil or negligible

(.) Estimate

.... Data not available.

As far as possibje, the basic concepts and terminology ured in the text are those giver in the Manual of Fiducational Stetistios published in 1961 by UIVESCO.

We trust that the present document will prove useful, not onto those Afghan and foreign specialists attached to the Ministry of Education, but also to all other experts interested in the problems of education in Afghanistan.

Henri MUTIER

Planning Team Leader 


\section{ON TENTS.}

UNESCO Planning Team in Afghanistan

Foreword

Introduction

Table 1. Mother tongues in lifghanistan

Table 2. European languages taught at secondary level idministrative divisions of Ligghanistan

Map

The organization of the State (Fig. I)

\section{Part L - Socio-economic background}

I Demographic data

1. Estimated total population

2. Estimater school age population

Table 3. Legal primary school age population (1964-1980)

3.. Distribution of population by provinces

Table 4.

II Economic development

1. Economic situation

Table 5. Major industrial production

2. Labour force

3. Gross National Product and National Income

Table 6. National Income estimates

Table 7. G.N.P. - 1960-1980

II Financial data

Table 8. Government expenditures and receipts (1962-1963)

Table 9. Domestic government expenditures (1960-1964) 
Organization of the educational system

Fig. 2. Summary flow diagram

Fie. 3. Organization chart of the Ministry

I. Schools

Teble 10 - Historical trend in number of schools 1932-1964

Table 11 - Number of schools in 1964

(Primary and Secondary levels)

Table 12 - Status of school bujldings in 1964

(Independent primery schools)

II Pupils (Frtmary and secondary levels)

1. Historicel tend in number of pupils (al1 levels) $\ldots \ldots \ldots \quad 27$

Table 13 - Historicel trend

Fiq. 4. Estimated increase of annual intake in grade I $(1958-1964)$

\section{Enrolment by sex and grade in 1964}

Fig. 5 - Educational pyramid in 1964 (all levels)

Table 14 - Primary level

Table 15 - Distribution of pupils by provinces

(irimary level - all schools)

Table 16 - Middle schools and lycees

Table 17 - Nidddle schools and lzcees in Tabul City

Table 18 - Vocational schools (excl. Teacher training)

Table 18 bis - Teacher Training schools

Table 19 - Vocational schools in Kabul City

3. Estimated enrolment rates in primery education

FIE. 6. - Increase between 1960 and 1964

Table 20 - Rates by Provinces (1964)

Fig. 7 - Map showing enrolment at primary level (1964) 
The Afghan sooiety is an Islamic society, mainly of the Hanafite sect, in which the traditional pattern of life is undergoing a gradual change. Since the late 19 th and early 20 th centuries the power of the large landlords and religious leaders has been gradually replaced by thet of the central Governmer

At the instigation of H.H. King Nohamed Zaher Shah a new constitution was prepared and - after having been discussed $b_{i}$ the High Assembly ("Loya Djirgah") - promulgated on the 1 st of October 1964.

The new Constitution inaugurates a Constitutional Monarchy with a Parliament, called "Shoura", consisting of two chambers, the "Wolossi Djirgah" electer by universal suffrage, and the "lashranon Djirgah", of which one third of the members are nominated by the King and two thirds are provincial representatives. Ethnically, the population of Afghanistan is mixed, but the Pakhtoons (Pashtoons), of indo-european origin, are in the majority. Several languages are spoken in the country, and their relative importance has been estimated as follows :

Table 1

Mother tongues in Afghanistan (1)

\section{Languages}

\% of population

1) Indo-european languages

a) Iranian :

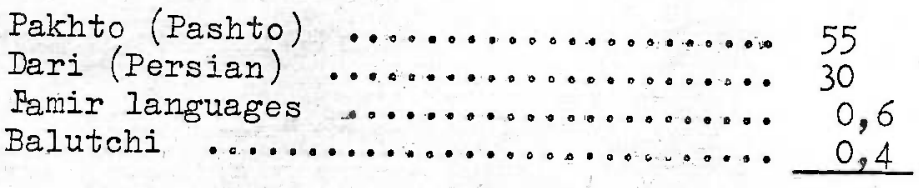

b) Dardic:

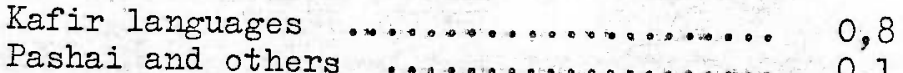

Pashai and others ............... 0,1

c) Indian :

Lahnda $\ldots \ldots \ldots \ldots \ldots \ldots \ldots \ldots \ldots \ldots \ldots \ldots \ldots \ldots \ldots \ldots$

2) Turkish languages

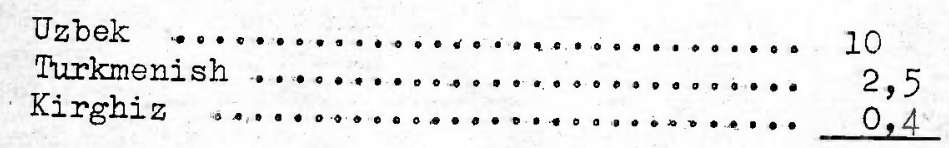

3/ Other languages

Dravidian (Brahoui), Mongol, Arabic ... $\frac{0,1}{100,00 \%} \frac{0,1}{\frac{0.1}{10}}$

(1) WILBER-Afghanistan-NEW-HAVEN, 1956 (cited by HUILUL - Geography of Afghanistan GYLDENDAI, COPEIVHAGEN, 1959 - P. 95) 
The two official languages are

Pashto and Dari. One of these is taught in grades I to III according to tho regions, and both from grade IV on.

The European languages which are taught at secondary level are distributed as follows : Table 2

European languages taught at secondary level

Total secondary level

(all types)

Iycees (Grades VII-XII)
English

$90 \%$

$88,9 \%$
French

$$
3,5 \%
$$

$7,1 \%$
German

$6,5 \%$

$4 \%$

The Constitution states that "education is the right of every Afghan and shall be provided free of charge by the State to the citizens of Afghanistan", that "the aim of the State in this sphere is to reach a stage where adequate facilities for education will be made available to all Afghans", and that "primary education is compulsory for all children in the areas where the facilities for this purpose are provided by the state".

The beginnings of a modern school-system were laid in 1904, when FABIBIA Lycee was founded. The British system in vogue in Indie was predominant at first, after which the French system was indirectiy introduced through Turkey, with whom Afghanistan established cultural agreements.

Cultural relations with ther countries developed fully as a resuli of the attainment of full National Independance in 1919.

Afghanistan has been a member of UNESCO since 1947, and the first international mission of educational experts came to the country in 1949 . Bilateral aid in the field of education is now mainly given by the United States of America, the Federal Republic of Germany, and France. Recently the Soviet Union and Afghanistan have signed an agreement which pledges the first to provide for the training of engineors and technicians.

To-day one of the most essential aims of the country is the development of a national educational system, closely adapted to its economic, social and cultural needs. It is with this goal in view that the Royal Govornment Authorities have devoted themselves since 1957, within the frame of the first (19571961) and the second (1962-1967) five-year plans.

The difficulties to be overcome are severe: shortage of teachers and funds, and also problems of communications, which render the establishment of schools difficult.

The Afghan economy is still a rural subsistence economy. It will be necessary to transform it gradually into a market economy, if general development is to have a solid basis. Education is one of the most important means to achieve this goal. 
The number of provinces has been increased in 1964. By this increase certain provinces which too large could be made smaller, this improving the officacy of administration.

As the annual survey of school-enrolment took place before the abovo mentioned roform, certain tables of this document apply to the old provinces.

In the following we give the relation-table of old and now administrative divisions :

\section{Old provinces}

KABUL-City

KABUL-Province

PARTILIT

BAIIIAIV

NANGARHAR

PAYTIA

GHAZII

KAINDAHAR

GRISEIK

TPARAE

HERAT

GHORAT

MATRIATIA

SHI BARGHAN

KATAGIXAN

BADAKHSHAIN

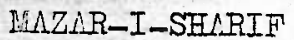
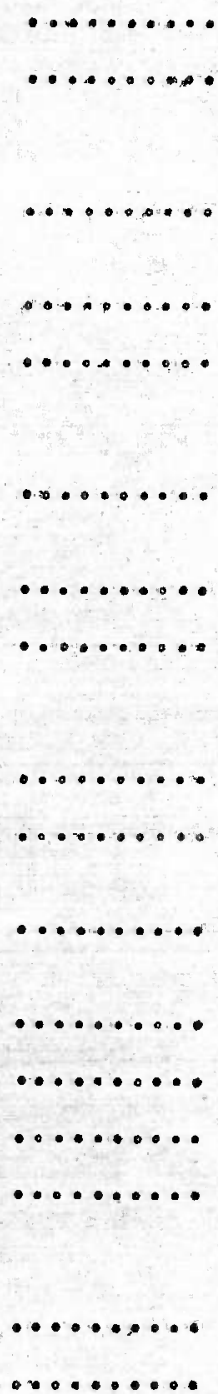

\section{Now provinces}

KABUL_City

IFABUL-Province

WARDAK (MAIDAIN)

IOGAR

\section{PARWAIV}

KAPISA

BAMI ANT

INANGARHAR

KUNARHA

IAGPMAN

PAKTIA

KATAWAZ and ORGOON

GHAZNI

KANDAHAR

ZABUL ( $\Lambda \perp A T)$

OROZGAN

HETMIND

FARAH

CHAKIIANSOOR

HERAT

BADGHISAT

GHORAT

FARYAB

JAWZJAN

KUNDUZ

BAGHLAN and PUI-I-KHUMRI TAKEAR (TAJOKAN)

BADAKHSHAN

BAIIKH

SAMANG $\triangle N$ 


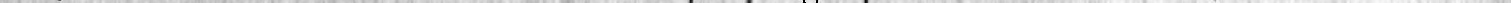


Part A.

Socio-Economic Background 


\section{DEROGRAPHIC DATA}

\section{1 - Estimated total population}

istimates of total population for the year $1960^{\circ}$ vary greatly from 8 millions to 13,8 millions.

For the purposes of the present study, we have adopted the estimate used in the report of the recent UNESCO Regional Educational Planning Mission to Afghanistan (January-February 1964) viz., 12300000 inhabitants in 1960 (1).

This choice does not imply that other estimates are rejectod outright, and it is cloar that all basic demographic data will need review, as more accurate information becomes available.

The generally accepted growth rate is $1,75 \%$.

Under these conditions, our working hypothesis for the 1964 population is 13200000 .

2 - Estimated school age population

Then studying the age pyramido of Iran (1960) and Pakistan (1961) we find alnost the same percentage ( $27 \%$ and $27,2 \%$ respectively) in both contries for the age groups 5-14 years, whioh is of the most direct interest for educational planning. (2). For present purposes the Iranian pyramid has been adopted, since, that of Pakistan covers markedly heterogenous populations of the Western and Eastern wings .

As in the UNISCO document already quoted (1), we have assumed the masculinity ratio (ratio men-women) $52 / 48$, or $\frac{52 \times 100}{48}=108$ men for 100 women (Iran 1960: 103 - Pakistan $1961: 111)$.

On. this basis it has been possible, by the SPRAGUE method (3), to arrive at an estimate of the "legal" primary school population (age group of 7-12 years) in 1964 and in 1980.

Table 3

legal primary school-age population (1964-1980) ( $x$ 1000)

\begin{tabular}{|c|c|c|c|c|}
\hline \multirow{2}{*}{1964} & $\frac{\text { Age }}{7}$ & $\frac{\text { Boys }}{20}=$ & Girls & Total \\
\hline & & $203=1$ & 187 & 390 \\
\hline & 8 & 197 & 181 & 378 \\
\hline & 9 & 191 & 176 & 367 \\
\hline & 10 & 186 & 171 & 357 \\
\hline & 11 & 181 & 167 & 348 \\
\hline & 12 & $176=$ & 162 & 338 \\
\hline & -12 & 134 & 1044 & 2178 \\
\hline
\end{tabular}


$1964=100$

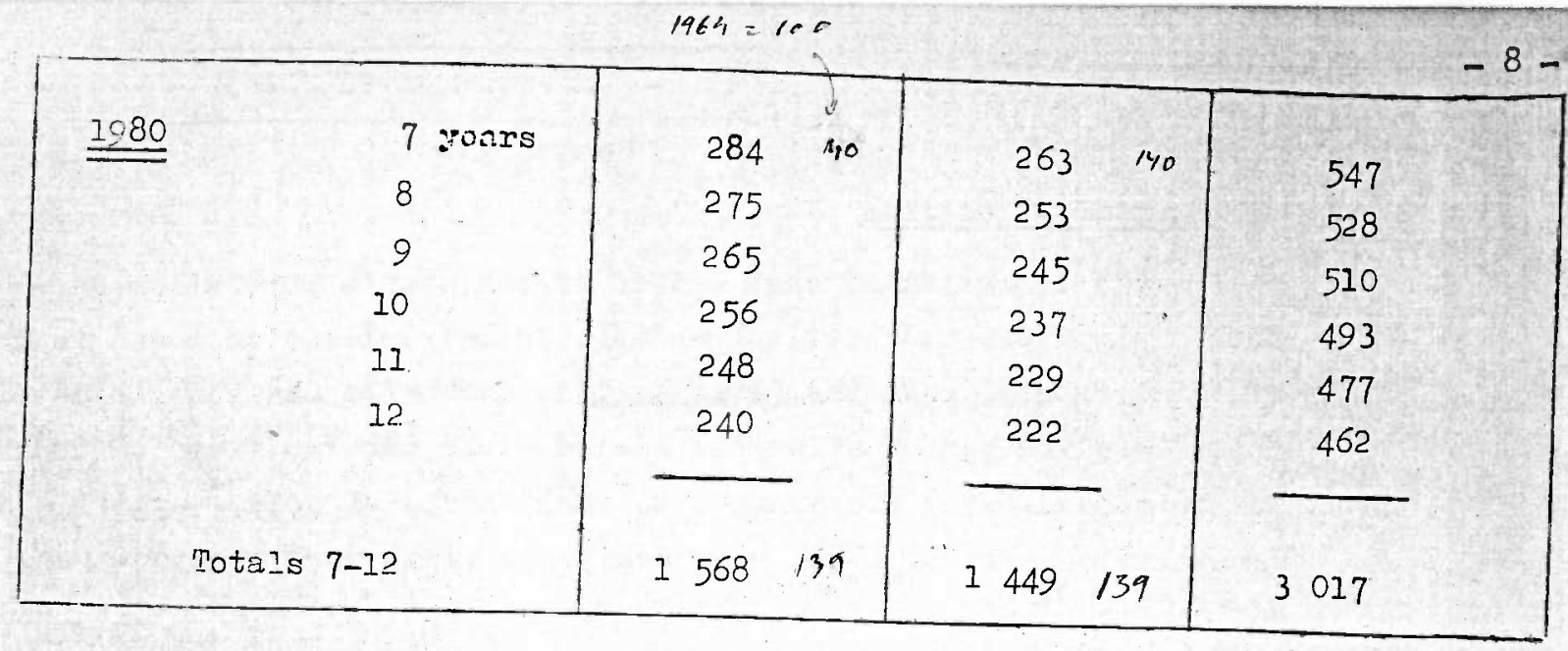

3 - Distribution of population by provinces

The linistry of Planning has made population estimates for the old provincial divisions (4). The results, as percentages of the total population, are as follows (nomads, estimated to be $2,400,000$ in 1960 , not included):

Table 4

\begin{tabular}{|c|c|c|c|c|c|}
\hline Provinces & ' & $\%$ & Provinces & & $\%$ \\
\hline $\begin{array}{l}\text { FABUL } \\
\text { (o1ty casd provin }\end{array}$ & $\ddot{c e j} \ddot{j}^{\cdots \cdots}$ & 12 & & $\cdots \ldots \ldots$ & 3,5 \\
\hline NAINGARHAP & $\ldots \ldots \ldots \ldots$ & 9,2 & OROZGAIN & $\cdots \ldots \ldots$ & 3,4 \\
\hline PARWAN & $\ldots \ldots \ldots$ & 8,7 & MAIMANA & $\ldots \ldots \ldots$ & 2,9 \\
\hline KAITDAH $/ \mathrm{R}$ & 0.00 .0 & 7,3 & SHI BARGAN & $\ldots \ldots \ldots \ldots$ & 2,6 \\
\hline KATAGHAIT & $\cdots \ldots \ldots, \ldots$ & 7,1 & $B A M \perp N_{1}$ & $\cdots \cdots \cdots$ & 2,3 \\
\hline HERAT & $\cdots \cdots$ & 7,1 & GHORAT & $\cdots \cdots \cdots$ & 2,3 \\
\hline PAKTIA & $\cdots \cdots$ & 5 & BADAKHSHAN & $\cdots \ldots \ldots$ & 2,1 \\
\hline IAZAR-I_CHARIF & $\ldots \ldots \ldots$ & 3,6 & $\begin{array}{l}\text { GRISHK } \\
\text { FARAH }\end{array}$ & $\begin{array}{l}\ldots \ldots \ldots \ldots \\
\ldots \ldots \ldots \ldots \ldots\end{array}$ & $\begin{array}{l}2 \\
1,3\end{array}$ \\
\hline
\end{tabular}

Sources of soction A I

(I) "Long-term Projections for Education in Afghanistan" (Preliminary Draft Report) UNSSCO Regional Advisory Team for Educational Planning in Asia, BANGKOK, April
1964

(2) Domographic Yearbook - 1962, United Nations

(3) "léthodes de projections démographiques par sexe et par âge", Manuel. III Nations-Unies, 1954

(4) "Basic statistics of Afghanistan" - Statistics and Research Departement, Ministry of Planning, KABUL, 1962 


\section{1) Economic situation}

It is estimated that $85 \%$ of Lfghanistan's population earns its livelihood from agriculture and cattle-breeding. Industrialisation began in the third decade of this century, when the first textile factories and power plants were established. In the period after the sccond-world war the tempo of industrialization was increased, when plants for the manufacture of soap, vegetable oll, sugar, wollen textiles, etc... and a cotton ginning plant were started. Real progress came in the first and second Five-Year-Plans, Sept. 1956miarch 1962 and Niarch 1962Narch 1967 respectively.

\section{a) Sourcos of power:}

The Govornment has fostered the development of electricity and other sources of power. After the completion of the Sarobi-Power-Plant (Kapisa Province), before the start of the first Five-Year-Plan, the Girishk-Power-Plant with a capacity of $3800 \mathrm{~kW}$ and the Ghori-Power-Plant (9000 KW) were completed. The second Pulikhumry-Hydroelectrie-Plant has a capacity of $7000 \mathrm{KW}$ and was constructed because the amount of electricity for the new industrial plants at Pulikhumry was not sufficient. The production capacity of the Arghandah-Power-House in Kandahar Province was raiscd to $14000 \mathrm{KW}$ to meet the increasing demand for power in this area. The Khan-Abad-Power-Plant (Kunduz-Province) was constmcted by the Cotton Company; it will be destined to satisfy the needs of the Kunduz and Khan-Libad regions. The Naghloo-Hydro-Electric-Plant (Nangarhar-Province) is the biggest project undertaken as yet in Afghanistan. Naghloo will have minimum output of $67000 \mathrm{KW}$ and a maximum of $90000 \mathrm{KW}$. The Jalalabad-Canal-Power-Plant (Nangarhar-Province), now under construction, will have an installed capacity of $11000 \mathrm{KW}$. Also survey and exploration work on the Mahipar-Hydro-Electric-Plant (Kabul-Province) is under way. This plant will have a capacity of $40000 \mathrm{KW}$ initially, and $60000 \mathrm{KW}$ when the second phase of construction is comploted.

b) Cement :

The production of cement is carried on in the Ghori-Cement-Factory (BaghlanProvince), in the Pulikhumry-Factory. (Baghlan-Province) and Djebel Serraj (Parwen Province).

\section{c) Textiles :}

The Gulbahar-Factory (Parvan-Province) is the biggest factory in Afghanistan, consisting of 47000 spindles and 1422 automatic weaving machines with sections for white and coloured fabrics, prints and waste products. The Umeed-Rayon-Plant in Kabul may produce up to $650000 \mathrm{~m}$. of rayon annually. A big wollen Textile-Plant was installed in Kandahar and a Margarine and Soap-Factory in Kunduz. This factory has an annual ginning capacity of 40000 tons of cotton, expressing 4500 tons of cotton seed oil and producing margarine and soap.

d) Fruit-Preservation :

The Kandahar Fruit-Preserving and Canning Plant commenced production during 
i) in considering replacement and in order that natural losses be fairly closely predicted from population tables, the census authorities should prepare a tabulation of occupations by age groups. In addition statistics of volontary and compulsory retirement from active work, should also bo tabulated.

ii) Nlthough the probable extension of domand for specific occupational groups is particularly difficult to estimate in a society experiencing the full irpact of technological advance, attempts should be made to maintain the proper balance between various categorios of skilled workars, tochnicians and engineers. To this ond :

a) The genoral trends in the economy should be made plain, on the basi of the best statistical information, by the authorities responsible for forecasting population and for preparing a comprehensible econc mic plan.

b) In appraisal should be made of the changes likely to take place in the relative demand for different classes of goods and services.

c) An analysis should be made of the possible changes in the relative domand for different typos of skills within each industry. In estimating demand in the higher levels, it should not be assumed that the ratio of the number of such positions to the total number of vorkers will remain unchanged. Experience in other countries shoul be a valuablo guide in this fiold.

d) In giving a picture of the total needs of a single industry or occupation, some distinction should be made between different types of activity in accordance with a general classification.

Par. 20. - Estinate of supply and demand in the field of technical and vocational education will only give general indications and many other factors should be taken into account by the competent authorities, in coning to a decision.

Estimates of Labour Force and concepts such as the participation rate of labour force wero unknown in Afghanistan before 1963. An attempt had been made in 1959 by the Royal finistry of Planning, to obtain from other Ministries and Departments information about their employees, Afghan and foreign. In 1958/59 a survey was taken by the Royal linistry of Planning with the advice of the Ministry of Mines and Industries to get information in 88 industrial undertakings about their staffing, but as the response was unsatisfactory the received data were not processed.

In 1958 an administrative machinery for collection of employment data was set up in the Royal Linistry of Planning, the Directorate of lijanpower. Further, an other institution was originated, the Nanpower Commission, with mombers of some of the important linistries with the Deputy Minister of Planning as the Chairman and the Director of the Lianpowor-Department as the Secretary:

On October 3rd 1959 an expert of I.I.O. arrived and acted up to November 8th 1964 as lianpower-idviser in the linistry of Planning, KABUL. 
On the arrival of this expert sovoral neeting of the llanpower-Commission were held in which it was decided that a fresh approach to the collection of manpower data was to be undertaken by the Royal Ministry of Planning.

The following collections about employment market information were undertaken :

a) Collection of Employment Varket Information in KLBUL, Sept. 1962

b) JiLiLABiD Enployment Narket Report, Warch 1963

c) HERAT Ianpower Situation Report, April 1963

d) QATHGHAN Enployment liarket Report, June 1963

e) GHAZNI Employnent Narket Report, June 1963

f) KMNDAHAR lianpower Situation Report, June 1963.

Tho distribution of workers in nine Urban Areas by Occupational Groups and Industrial Divisions is given in Innex 24, in Survey of progress, 1962-1964, published by the Royal Iinistry of Planning, Department of Statistics and Research, KabuI, Sombola 1343 (August 1964). The figures in these reports are given according to the ISCO (International Standard Classification of Occupations).

In 1958/59 it wes docided by the Government that information should be collected and maintained on a nation-wide besis in respect of the highly educated and trained persons in the country. A National Register for Specially Trained Personnel was set up in the Nanpower Directorate of the linistry of Planning. It included graduates of Technical Institutions in Kabul, Kandahar and Khost.

In 1961 information about the Ifanpower-requirements for the Second-FiveYear Plan was collected by the liappower Directorate in the linistry of Planning. Tho figures by broad occupational groups according to ISCO are available in the Lianpower Directorate.

Furthermore in August 1964 a standard from was sent out to all authorities who were engaged in the above mampower projections for the Second-Five-Year-Plan, to study the number of jobs actually created as compared with the original projections. This information will be available in ashort time in the lianpower Directorate of the Ministry of Planning.

In 1963 an Employment Service under the Department of Labour in the Hinistry of lines and Industries was set up. It is intented to open additional Employment-Service-Offices in Kandaher, Herat and Nazar-i-Sharif in the Third-Five-YearPlan-Period.

A schome for the resettlement of workors becoming surplus in different projects of the Plan after the completion of works was urgently needed. In a meeting of ropresentatives of the Ministries of Planning, Mines and Industries and Public Works, it was agreed that the lianpower Directforate should collect advance information relating to the anticipating lay-offs and also, relating to the job opportunities arising in different fields. This information would be sent to the Employment Office in Kabul to arrange for resettlement of these workers.

The distribution of University and technical graduates will be arranged corresponding to a procedure approved by the linistry of Planning. The preliminary data required for arranging the distribution will be collected by the lwanpower 
For Educational and Vocational Guidance the Ninistry of Education set up in July 1964, a Directorate of Vocational Guidance. Information was collected by this institution about wages, hours of work and qualifications of workers by thi I.I.0. Expert in the Lanpower Directorate of the Hinistry of Planning and passec on to the Diroctorate of Guidance for its use. In addition a study of the employront histories of the graduatos of the yoars 1336,1337 and $1338(1957,1958,1955$ of ifghan Institute of Technology, Kabul, and Niechanical School, Kabul, was organised. On Octobor 1st 1964 a first "Guidance Leeting" for the students of Liechanic School, Kabul, was arranged, in which representatives of different undertakings addressed the students. Iioro of these "Guidance Neetings" will be organised in the future.

Accorcing to Survey of Progress 1962-64, linistry of Planning, August 1964, the following shows industrial omployment in 1963 (preliminary ostimates) for es b lishments omploying more than 20 persons :

Number of establishments interviewed

Coal-lisining

Non-jictallic-ilining or Quarrying

Food-Lanufacturing

Textilo Manufacturating

Furniture and Wood llanufacturing

Printing and Publishing

Briquetting of Coal

Lianufacture of Non-lietallic Products

Ropair of Transport Equipment

Uiscollanous Lanufacturating

Electricity

Toxtiles is by far the largest industry in the country, accounting-for more than 50\% of the total industrial labour enployed. It employod about 11000 in 1963 lining and quarrying, including coal mining, food manufacturing, printing, manufacturating of non-netal products and repair of transport equipnent and other large employers, each employing more than 1000 persons on the average. Furniture and woo manufacturing is the next industry in order of employment, employing 800 in 1963 , followed by clectricity. Concerning the employment in Govermment Services in Afgha nistan, the numbor of persons omployed in Government-Services in 1963 (excluding the development projects and the armed forces) was ostinated to bo as follows:

Lamurs $\quad 17500$

Ifustakhdims 32000

For dotails of ranks soe table Annex 25 of Survoy of Progress, 1962-1964, linistry of Planning, August 1964.

The omployment on major development projects in 1963 was as follows, according to Survey of Progress, 1962-1964, Iinistry of Planning, August 1964 : 
Project

Kush-Horat-Kandahar Road

Salang Tunnol

Ja?alabad Canal

Naghiso Hydroclectric Plant

Bridgos Project in Nangarhar

Shindad Airport

Serdeh Dam

Oil and Gas Ixploration

Iinoral Bxploration

Silo at Puli-Khumri

Kabul-Kandahar road

Sher Khan Port

Pul-i-Charkhi Worsishop
W orkers 1963

11000

10123

13800

7200

1808

1649

920

2150

430

76

2876

490

2967

55559

\section{3-GrossNational Product and National Income}

National Income is not regularly measured in Afghanistan, and thero aro major gaps - particularly in agricultural production and output of handicraft industries - which means that any estimate made at this time is extremely rough. Table r resumes all National Income estimates that have been made at one time or another for Afghanistan. (Estimatos A, C and D are by far the most detailed. Each of these three estimates is supported by from 25 to 40 detailed tables on individual sectors and components).

Table 6

National Income estimates

(in billions of afghanis)

\begin{tabular}{|lllllll|}
\hline $1332(1953 / 54)$ & A & B & C & D & I & F \\
$1335(1956 / 57)$ & & 12,5 & & & & \\
$1337(1958 / 59)$ & & & & & & \\
$1339(1960 / 61)$ & 30,4 & & 42,6 & 29,6 & & \\
\hline
\end{tabular}

Principal sources : linistry of Planning, Department of Statistics and research (Among others : "Gross National Product for Afghanistan" - 1337) 
In the roport "Long-term projections for education in Afghanistan" cited page 8, the following estimates of Gross National Product from 1960 to 1980 are assumed :

\section{Tabie 7}

Gross liational Product 1960-1980

(based on 1960-61 prices)

\section{Year}

Rate of Growth

$3 \%$

$4 \%$

(billions of afghanis)

1960

30

30

1965

35

37

1970

40

44

1975

47

1980

54

54

66 


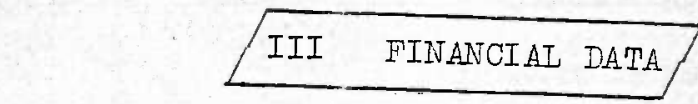

Table 8

Government expenditures and receipts : 1962-1963 (1)

(in millions of afghanis)

$\underline{1962}$

Expenditure

Development

Ordinary

Total

Revenue

Gove revenues

Commodity assistance

Foreign assistance, economic and technical

Deficit financing

Total
(\%)

$4523 \quad(71 \%)$

$1850 \quad(29 \%)$

6373
$51(0,8)$

(3) $3081 \quad(48,3)$

$1058 \quad(16,6)$

6373
1963

(2)

\section{(\%)}

$\begin{array}{ll}4928 & (68,4 \%) \\ 7272 & (31,6 \%)\end{array}$

$3137 \quad(43,6)$

$183(2,5)$

$3323(46,2)$

$557 \quad(7,7)$

7200

(1) To be exact : liferch 1962-March 1964 (Nuslim calendar : 1341-1342).

(2) Figures for 1963 are preliminary estimates only.

(3) Total amount of foreign assistance was assumed equivalent to 734 million U.S. dollars convertible at the exchange rate prevalent at the time of the formulation of the Plan (32.35 Afs $=1 \mathrm{U} . \mathrm{S}$. dollar). Actual rate: official = 45 Afs $-f r e \theta=63.5$ Afs.

Source : "Survey of Progress (1962-1964)" - liinistry of Planning, Department of statistics and research - KABUL, 1964 - 
Table 9

Domestic government expenditures

\begin{tabular}{|c|c|c|c|c|c|c|c|c|}
\hline (in millions of afghanis) & \multicolumn{2}{|c|}{1960 (1) } & \multicolumn{2}{|c|}{$1962^{(2)}$} & \multicolumn{2}{|c|}{$\begin{array}{c}1963^{(2)} \\
\text { preliminary) }\end{array}$} & \multicolumn{2}{|c|}{$\begin{array}{l}1964^{(2)} \\
\text { (budget) }\end{array}$} \\
\hline Foreign debt service & 179 & & 258 & & 512 & & 401 & \\
\hline Defense & 438 & & 594 & & 598 & & 567 & \\
\hline Finance & 97 & & 12.7 & & 156 & & 123 & \\
\hline Fiducation & 159 & 7,6 & $\underline{240}$ & 7,3 & $\underline{309}$ & $\underline{8}$ & $\underline{302}$ & 1 \\
\hline Intorior & 108 & & 131 & & 150 & & 143 & \\
\hline Foreign Affeirs & 27 & & 85 & & 66 & & 72 & \\
\hline Health & 37 & & 48 & & 62 & & 69 & \\
\hline Public Works & 67 & & 53 & & 53 & & 97 & \\
\hline Agriculture & 43 & & 45 & & 41 & & 52 & \\
\hline wines and Industries & 25 & & 42 & & 50 & & 51 & \\
\hline Tribal Lffairs & 41 & & 21 & & 25 & & 25 & \\
\hline Communications & 38 & & 33 & & 34 & & 36 & \\
\hline Other Ministries & 135 & & 136 & & 185 & & 223. & \\
\hline Helmand Valley & $\cdots$ & & 37 & & 31 & & 33 & \\
\hline Pay Raise and Contingoncies & - & & -- & & - & & 187. & \\
\hline Total Ordinary & 1394 & 66,4 & 1850 & 56,2 & 2272 & 58,6 & 2381 & 55,4 \\
\hline DEVSIOPMIIT & & & & & & & & \\
\hline Public Works & 378 & & 791 & & 774 & & 825 & \\
\hline Wines and Industries & $11 \overline{3}$ & & 367 & & 438 & & 460 & \\
\hline Dducation & $\underline{48}$ & $\underline{2,3}$ & 82 & 2.5 & $\underline{98}$ & $\underline{2,5}$ & 150 & 3,5 \\
\hline Agriculture & 20 & & 32 & & 38 & & 65 & \\
\hline Health & 16 & & 17 & & 11 & & 20 & \\
\hline Helmena Walley & 62 & & 69 & & 79 & & 60 & \\
\hline Civil Aviztion & 38 & & 44 & & 6 & & 10 & \\
\hline Comranications & 18 & & 9 & & 5 & & 53 & \\
\hline Press & - & & 10 & & 77 & & 86 & \\
\hline Rurel Development & 10 & & 14 & & 18 & & 20 & \\
\hline Finance & 3 & & 5 & & 51 & & 93 & \\
\hline Other linistries & - & & - & & 9 & & 17 & \\
\hline Contingencies & - & & - & & - & & .55 & \\
\hline Total Devolopment & 706 & 33,6 & 1442 & 43,8 & 1604 & 41,4 & 1914 & \\
\hline Grand Totel & 2100 & & 3292 & & 3876 & & 4295 & \\
\hline
\end{tabular}


1 - At KABUL, only grades 10 to 12.

2 - Next year, the courses will be extended to grade 13 included.

3 - Next year, these courses will be extended to grade 10 included.

4 - The two institutions are under establishment. The "Technicum" will prepare technets, while the Polytechnic school will form engineers.

5 - Preparation is principally aimed at administrative and diplomatic careers.

6 - Preparation is principally aimed at judicial careers.

7 - L = preparatory year common to faculties of Science, liedicine and Pharmacy during which courses deal with Nathematics, Physics, Chemistry and Biology (LPCB).

8 - The 5 th year in the faculty of Nedecine is divided between internship in hospitals and courses in the Faculty.

9 - A second faculty of Ved cine has been established in DJALAIABAD in September 1963. Courses are given in Pashto.

10 - The 4 th year will begin in 1965.

11 - Two sections : Agriculture - Veterinary medicine.

12 - Unrelated to the University - Attached to the Department of Vocational Education.

\section{General remarks :}

Primary classes are frequently attached to Lycees, Middle and Vocational schools. Iimilarly, Iycees have Eiddle level classes (grades VI to IX). 
Y- Wastage at primary level

Fig. 11

Cohorts beginning in 1958 and 1959

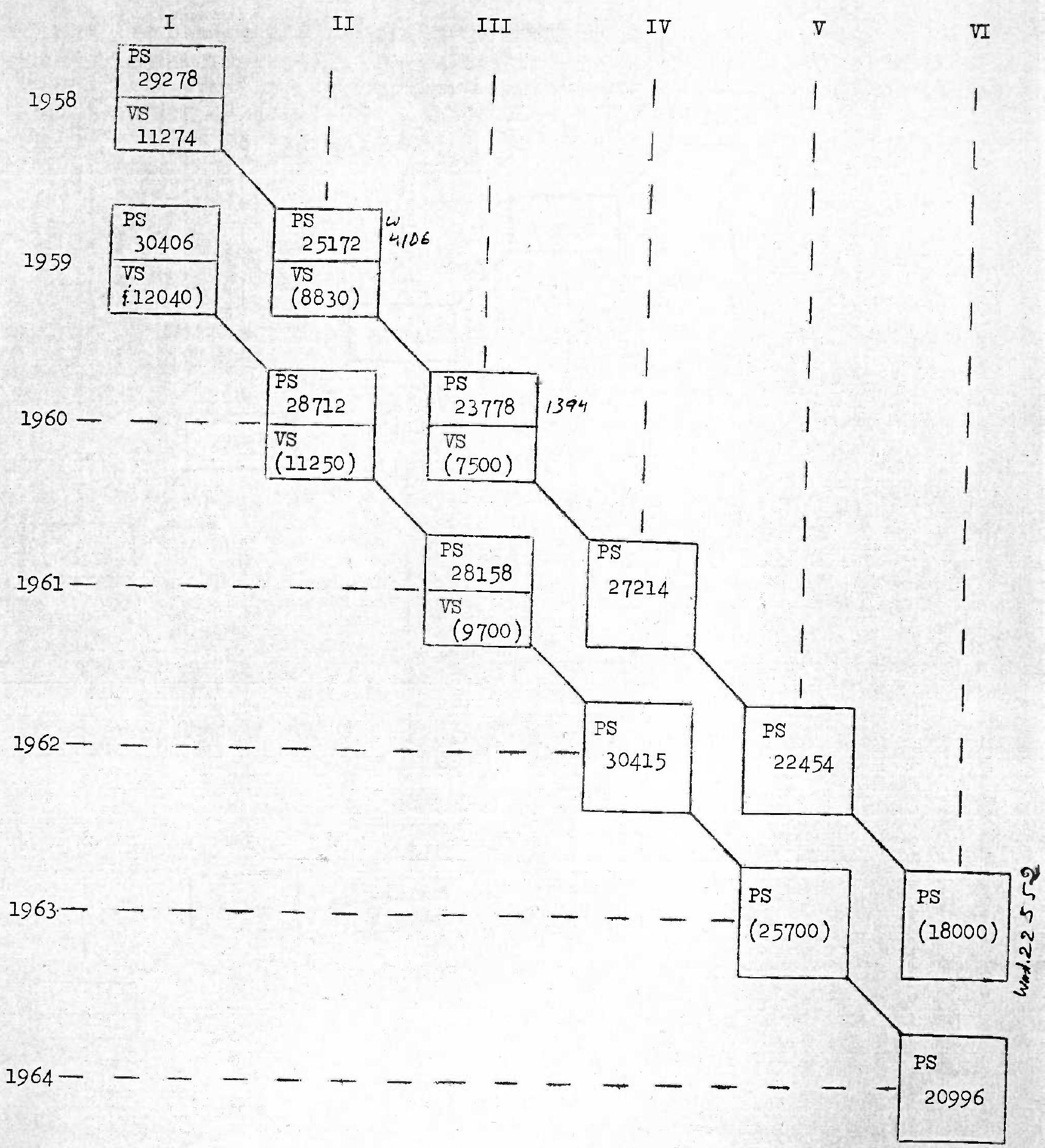

PS : Primary schools (incl. primary classes attached to secondary and vocational schools)

VS : Village schools 


\section{I $\mathrm{SCHOOIS}$}

1 - Historical trend in number of schools (1932-1964)

Sable 10

\begin{tabular}{|c|c|c|c|c|c|c|c|}
\hline Year & $\begin{array}{l}\text { Village } \\
\text { schools }\end{array}$ & $\begin{array}{l}\text { Primary } \\
\text { schools }\end{array}$ & $\begin{array}{l}\text { Middle } \\
\text { schools }\end{array}$ & I,ycees & $\begin{array}{l}\text { Vocationel } \\
\text { schools }\end{array}$ & Faculties & Total \\
\hline 1932 & - & $\cdots$ & $\infty$ & $\cdots$ & $\cdots$ & $-\infty$ & $2^{2}$ \\
\hline 1940 & - & $\cdots$ & $\cdots$ & $\cdots$ & $\cdots$ & $\cdots$ & 324 \\
\hline 1945 & - & $\cdots$ & $\ldots$ & $\ldots$ & $\ldots$ & $\cdots$ & 346 \\
\hline 1950 & - & 308 & 25 & 16 & 19 & 5 & 373 \\
\hline 1951 & 42 & 307 & 25 & 16 & 23 & 6 & 419 \\
\hline 1952 & 177 & 307 & 24 & 17 & 22 & 6 & 553 \\
\hline 1953 & 285 & 314 & 24 & 17 & 23 & 6 & 669 \\
\hline 1954 & 338 & 315 & 25 & 17 & 26 & 6 & 727 \\
\hline 1955 & 358 & 330 & 27 & 17 & 24 & 6 & 762 \\
\hline 1956 & 384 & 350 & 27 & 16 & 21 & 8 & 806 \\
\hline 1957 & 457 & 359 & 28 & 14 & 28 & 10 & 896 \\
\hline 1958 & 465 & 367 & 29 & 15 & 27 & 10 & 913 \\
\hline 1959 & 614 & 400 & 33 & 16 & 30 & 11 & 1104 \\
\hline 1960 & 695 & 456 & 40 & 19 & 28 & 12 & 1250 \\
\hline 1961 & 788 & 533 & 52 & 22 & 31 & 10 & 1436 \\
\hline 1962 & 820 & 542 & 60 & 24 & 34 & 11 & 1491 \\
\hline 1963 & 969 & 568 & 83 & 25 & 53 & 11 & 1709 \\
\hline 1964 & 1037 & 627 & 111 & 28 & 57 & 11 & 1871 \\
\hline
\end{tabular}

3E Incl. Teacher Training Schools

Note: It was hoped to have these global figures further analysed according to the importance of the establishments. But, nfortunatily, the necessary relevant datr. are not available at present. 
Table 11

Number of schools in 1964 (Primary \& secondary levels)

\begin{tabular}{|c|c|c|c|c|c|c|c|c|c|c|}
\hline \multirow[t]{2}{*}{ Provinces } & \multicolumn{2}{|c|}{$\begin{array}{l}\text { Village } \\
\text { schools }\end{array}$} & \multicolumn{2}{|c|}{$\begin{array}{l}\text { Primary } \\
\text { schools }\end{array}$} & \multicolumn{2}{|c|}{$\begin{array}{l}\text { Middle } \\
\text { schools }\end{array}$} & \multicolumn{2}{|c|}{ Lycees } & \multicolumn{2}{|c|}{$\begin{array}{l}\text { Vocational } \\
\text { schools }\end{array}$} \\
\hline & Boys & Girls & Boys & Girls & Boys & Girls & Boys & Girls & Boys & \\
\hline FABUL-City & - & - & 35 & 23 & 9 & 6 & 8 & 4 & 12 & \\
\hline ILABUL-Province & 57 & 12 & 51 & 5 & 9 & - & - & - & 1 & \\
\hline KAINDAHAR & 79 & - & 41 & 4 & 9 & 1 & 2 & 1 & 2 & \\
\hline $\mathrm{H} \Psi \mathrm{R} \Lambda \mathrm{T}$ & 56 & 7 & 43 & 8 & 4 & 1 & 2 & 1 & 6 & \\
\hline IHAZAR-I-SHARIF & 63 & 6 & 30 & 6 & 4 & 1 & 1. & 1 & 2 & \\
\hline KATAGHAN & 38 & 3 & 25 & 6 & 4 & 3 & 1 & -- & 5 & 1 \\
\hline NLING ARHAR & 80 & 21 & 52 & 6 & 9 & 1 & 1 & - & 8 & - \\
\hline PAKTIA & 44 & - & 54 & 2 & 7 & - & 1 & - & 3 & \\
\hline PARWAN & 37 & 12 & 24 & 3 & 7 & 1 & 1 & - & 1 & \\
\hline GHAZNI & 49 & 6 & 30 & 4 & 7 & 1 & 1 & - & 1 & - \\
\hline GRISHK & 50 & 2 & 22 & 1 & 3 & 1 & 1 & - & 1 & - \\
\hline FARAH & 51 & 6 & 19 & 3 & 2 & 1 & 1 & - & 2 & \\
\hline EAINANA & 44 & 18 & $1 \varepsilon$ & 5 & 3 & 2 & 1 & - & 4 & -- \\
\hline BADAKHSHAN & 69 & 6 & 18 & 2 & 4 & 1 & - & - & - & 1 \\
\hline SHIBARGIINN & 46 & 4 & 12 & 4 & 3 & - & - & - & 2 & 1 \\
\hline BADIIN & 29 & 7 & 13 & 1 & 2 & - & - & - & - & - \\
\hline GHORAT & 31 & 9 & 18 & 1 & 1 & - & - & - & 1 & - \\
\hline OROZG IN & 28 & - & 10 & 1 & 2 & - & - & - & - & - \\
\hline TAIOKAIN & 32 & 9 & 15 & 1 & 1 & - & - & - & 1 & \\
\hline KAPISA & 19 & 7 & 9 & 2 & 1 & - & - & - & 1 & -- \\
\hline TOTALS & 902 & 135 & 539 & 88 & 91 & 20 & 21 & 7 & 53 & \\
\hline
\end{tabular}




\section{Table 12}

Status of school buildings in 1964

(Independant primary schools)

- in percentage -

\begin{tabular}{|c|c|c|c|c|}
\hline \multicolumn{2}{|c|}{ SIETE OWLED } & \multicolumn{3}{|c|}{ FON_EDATE OWNED } \\
\hline $\begin{array}{l}\text { School } \\
\text { buildings }\end{array}$ & $\begin{array}{l}\text { Mon-scinool } \\
\text { buildings }\end{array}$ & Rented & Free & Nosque \\
\hline $22 \square$ & $26,5 x$ & $26,5 \%$ & $15 \%$ & $10 \%$ \\
\hline \multicolumn{2}{|c|}{43,5} & \multicolumn{3}{|c|}{51,5} \\
\hline
\end{tabular}


(Primary and Secondary levels)

I - Historical trend in number of pupils

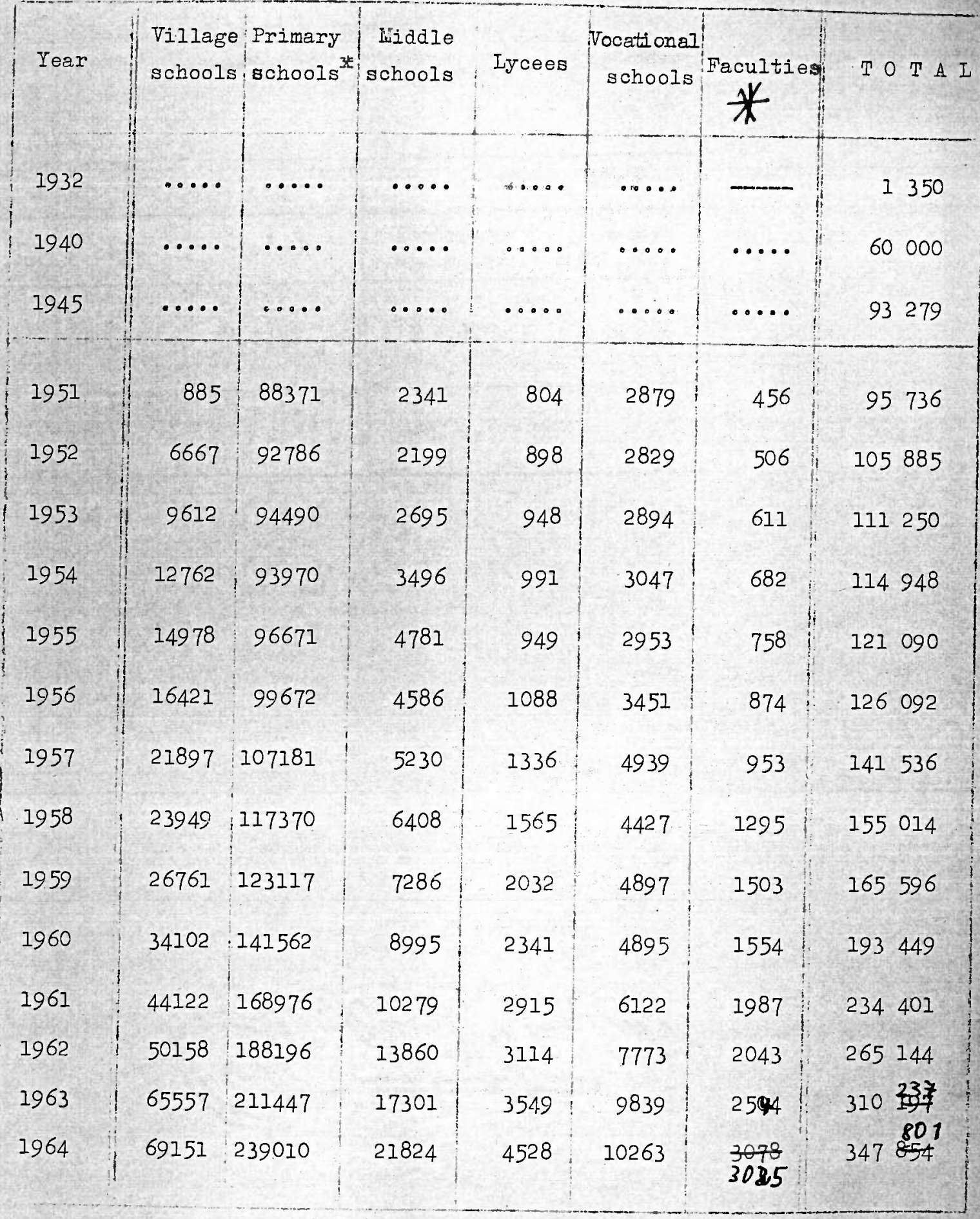

3* incl. primary classes attached to secondary or vocational schools 
Fig. 4

Estimated increase of annual intake in grade I between 1958 and 1964

INumber of

new pupils

$(B+G)$

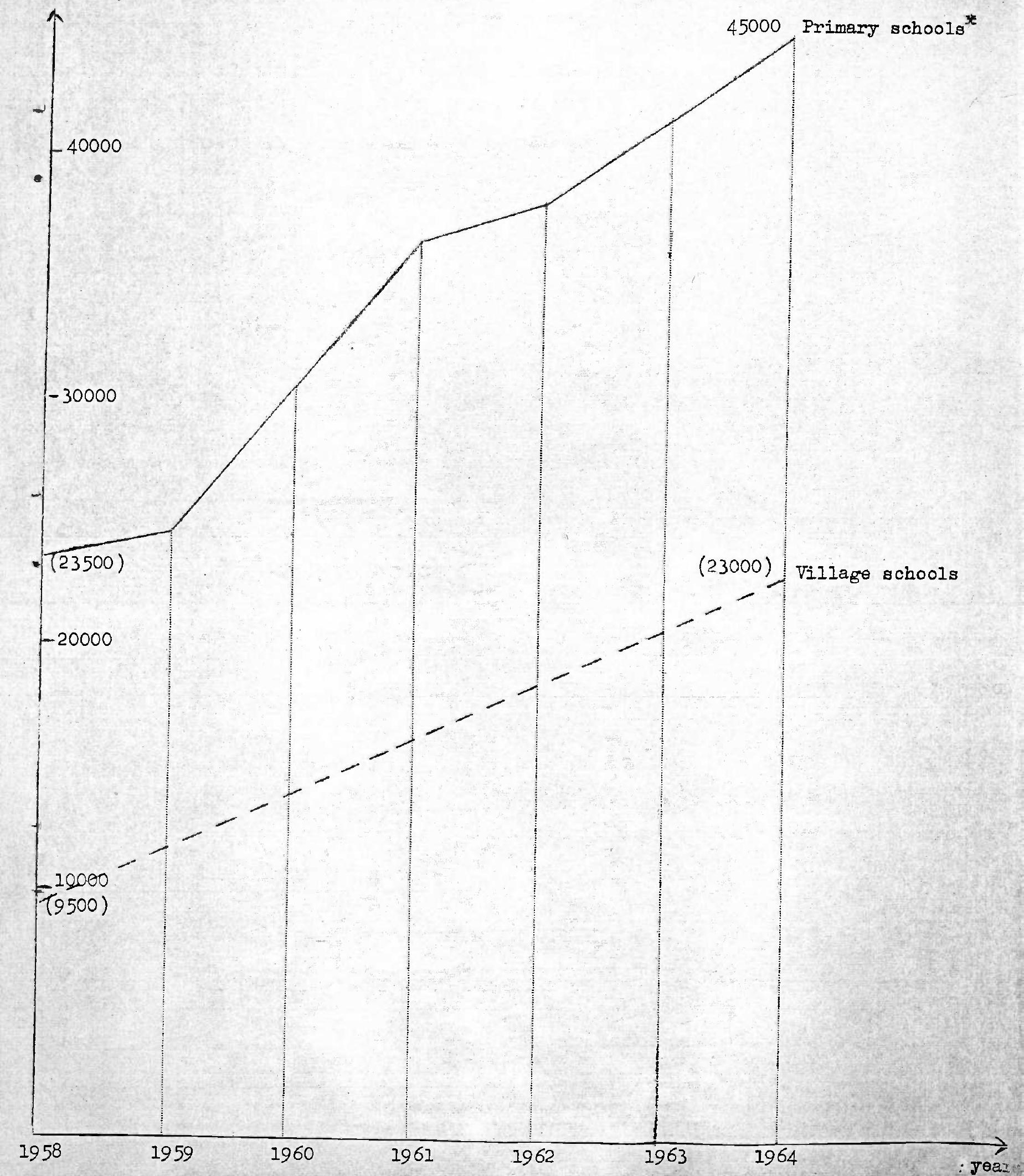


2. Enrolment by sex and grade in 1964

\section{Fig. 5}

Educational pyramid in 1964 - All levels -

Fatios

$$
\begin{aligned}
& \text { Secondory/fimary }=12 / 100 . \\
& \text { Higher/Frimary }=1 / 100
\end{aligned}
$$


Table 14

Primary level (1964)

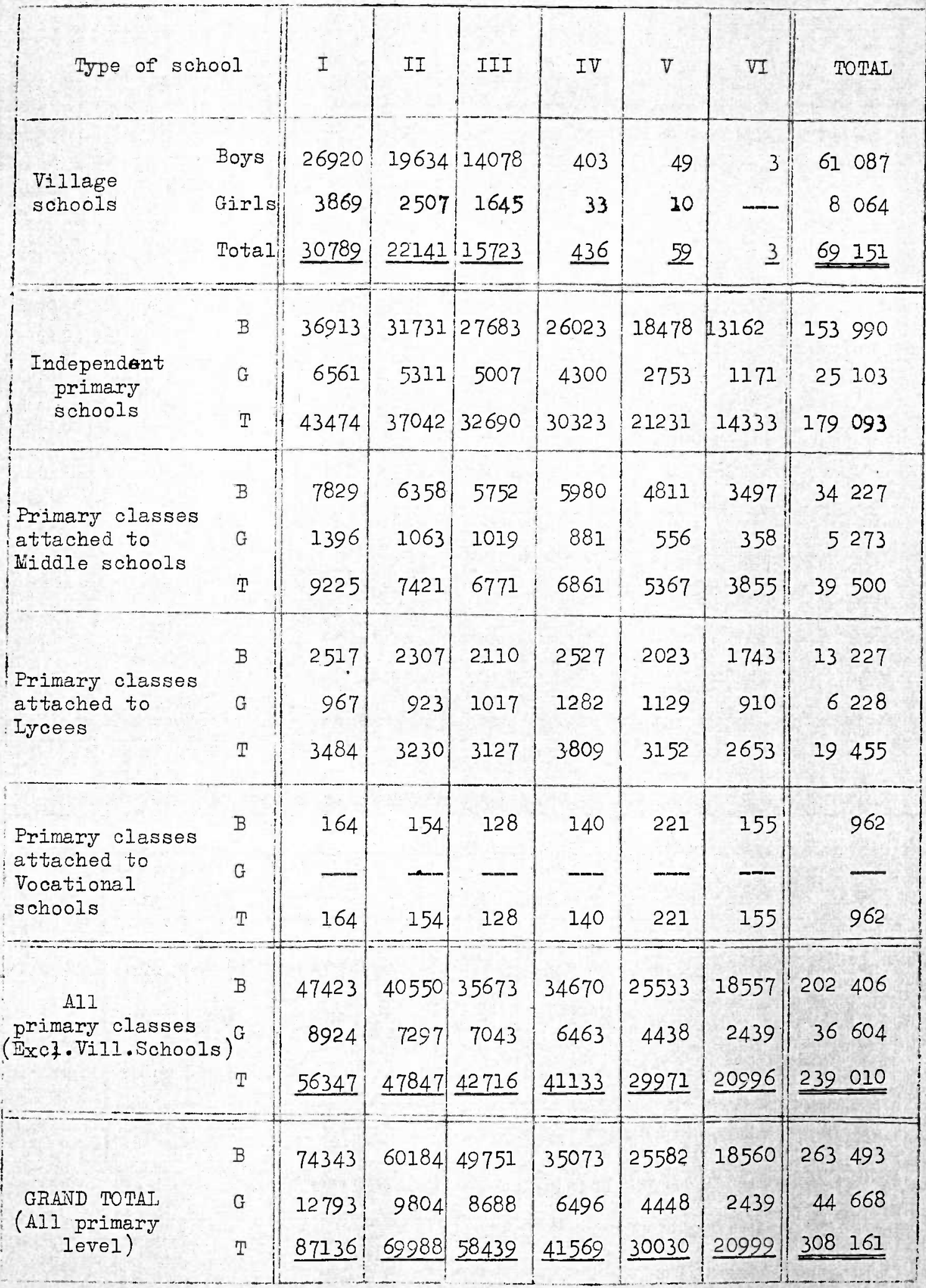

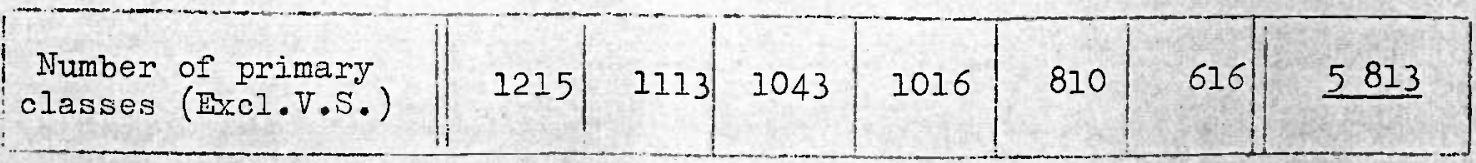


Table 15

Distribution of pupils by provinces in 1964

(Primary level - All schools)

\begin{tabular}{|c|c|c|c|c|c|c|c|}
\hline PROVIICES & Boys & Girs & Total & PROVINCES & Boys & Girls & Total \\
\hline KABUL-City & 33031 & 17651 & 50682 & KITAGHAIN & 13469 & 2594 & 16063 \\
\hline KABUL-Province & 25310 & $22 C_{7}$ & 27514 & TALOKAIN & 7886 & 750 & 8636 \\
\hline FARAH & 7925 & $8=6$ & 8741 & NANG $\triangle R H A R$ & 29152 & 3079 & 32231 \\
\hline BADAKHSHAN & 10628 & 1085 & 11713 & HERAT & 16906 & 2968 & 19874 \\
\hline MAZAR-I-SHLRIF & 15644 & 3029 & 18673 & GHORAT & 5143 & 433 & 5576 \\
\hline GRISHK & 9325 & $3 \div 8$ & 9653 & KANDAHAR & 14756 & 1423 & 16179 \\
\hline SHIBARGHAIN & 9624 & 1192 & 10816 & PARTAN & 11361 & 1481 & 12842 \\
\hline PAKHTIA & 18684 & $1 \in I$ & 18845 & KAPISA & 4878 & 804 & 5682 \\
\hline GHAZNI & 11701 & 874 & 12575 & BAEIAN & 3281 & 439 & 3720 \\
\hline MAIIIATINA & $1122 \%$ & $3297 !$ & 14526 & OROZGAN & 3560 & 158 & 3718 \\
\hline
\end{tabular}

Te.ble 16

Secondary level (1964)

- Liddle schocls and lycees -

\begin{tabular}{|r|r|r|r|r|r|r|r||c||c|}
\hline Boys & VII & VIII & IX & $\begin{array}{r}\text { Tut. } \\
\text { VII-I }\end{array}$ & X & XI & XII & $\begin{array}{c}\text { Tot. } \\
\text { X-XII }\end{array}$ & $\begin{array}{c}\text { TotaI } \\
\text { VII-XII }\end{array}$ \\
\cline { 2 - 3 } & 2120 & 1232 & 797 & 4149 & 509 & 348 & 213 & 1070 & 5219 \\
\hline Total & 10750 & 6701 & 4373 & 21824 & 2262 & 1276 & 990 & 4528 & 26352 \\
\hline
\end{tabular}

\begin{tabular}{|c|c|c|c|c|c|c|c|c|c|}
\hline $\begin{array}{c}\text { Number } \\
\text { of classes } \\
\text { (sections) }\end{array}$ & 262 & 192 & $\therefore 25$ & $\underline{579}$ & 66 & 43 & 33 & 142 & $\underline{721}$ \\
\hline
\end{tabular}

Table 17

Fiddle schools and lycees in KABUL-City

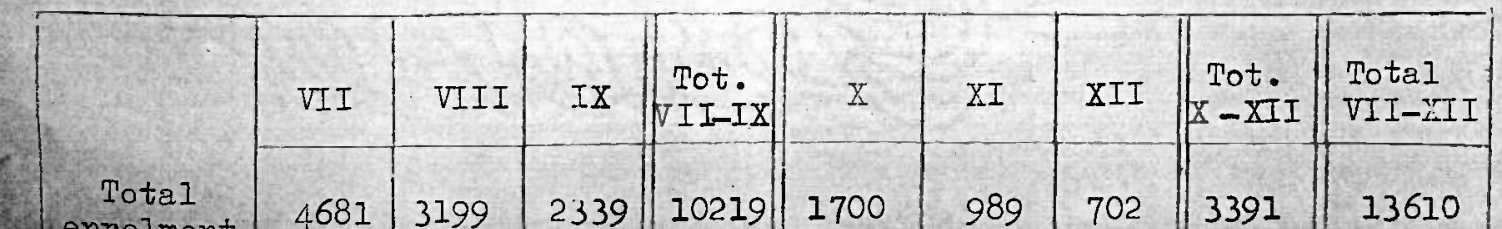


Seconders level (1964) - Vocational schools (excl. Teacher Training)

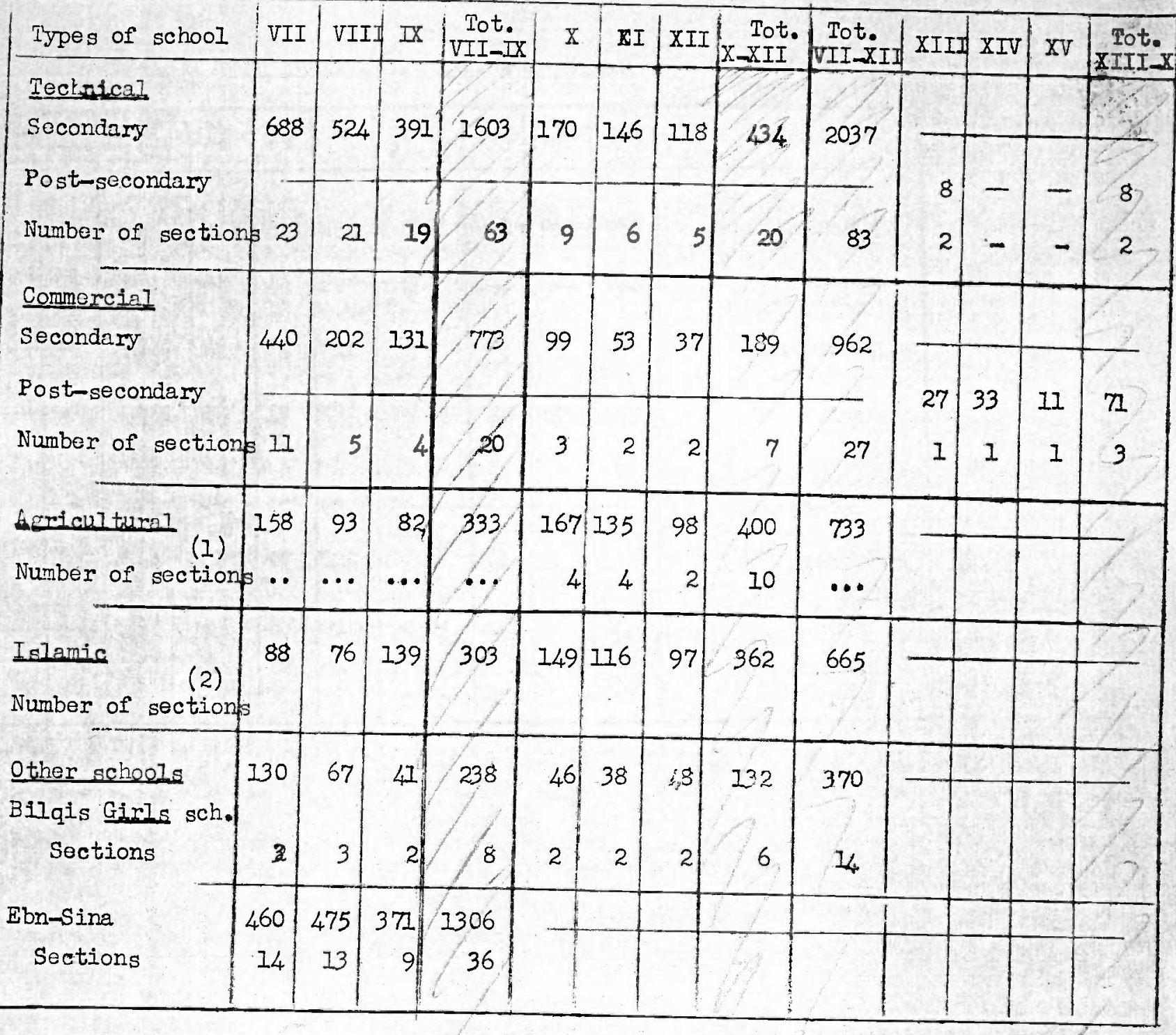

(1) KabuI Only - (2) Not available

Table I8 bis - Vocational schools: Teacher training

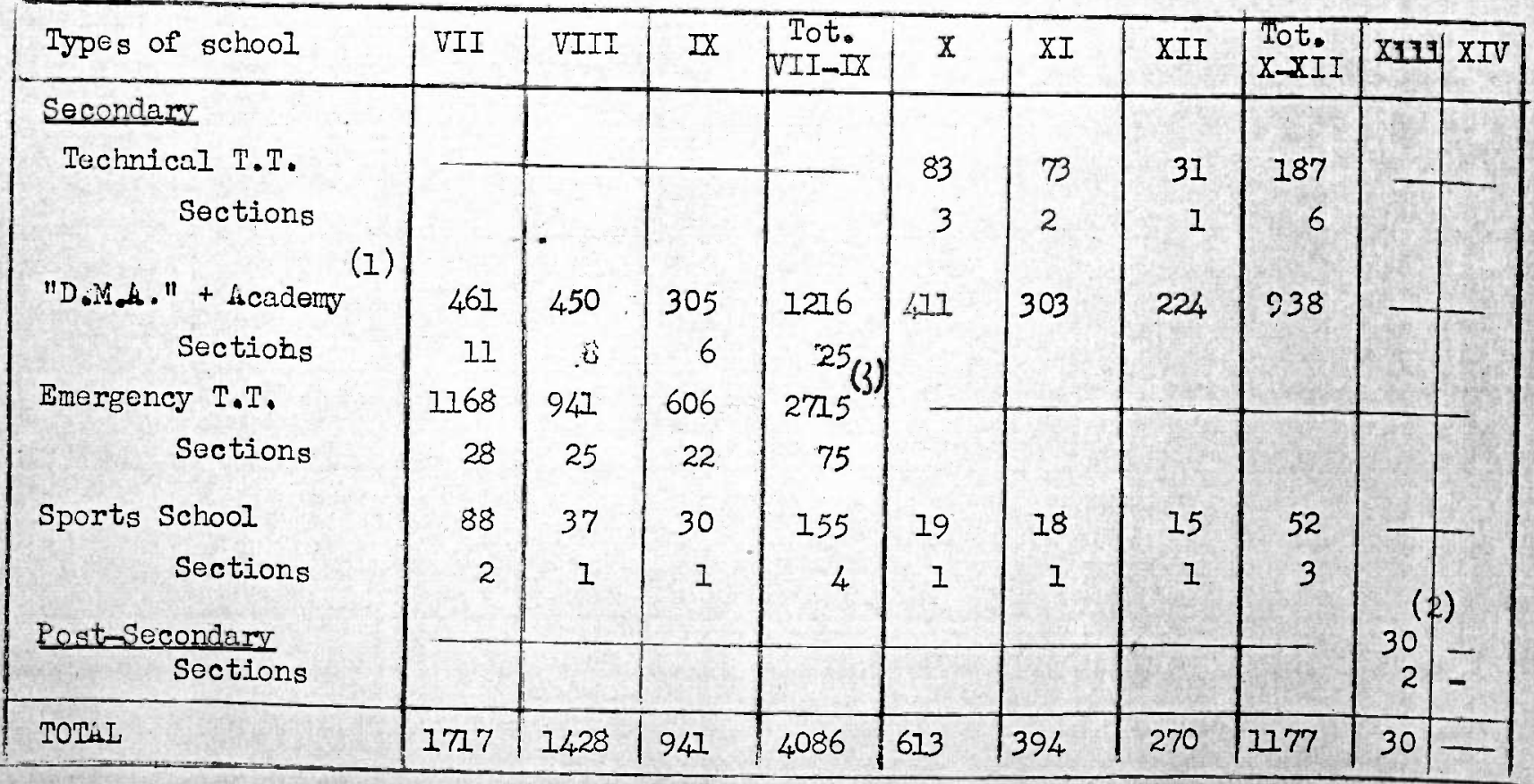


3 - Estimated enrolment rates in primary education (in relation to the estimated Fig. 6

Increase between 1960 and 1964

\section{Enrolm.}

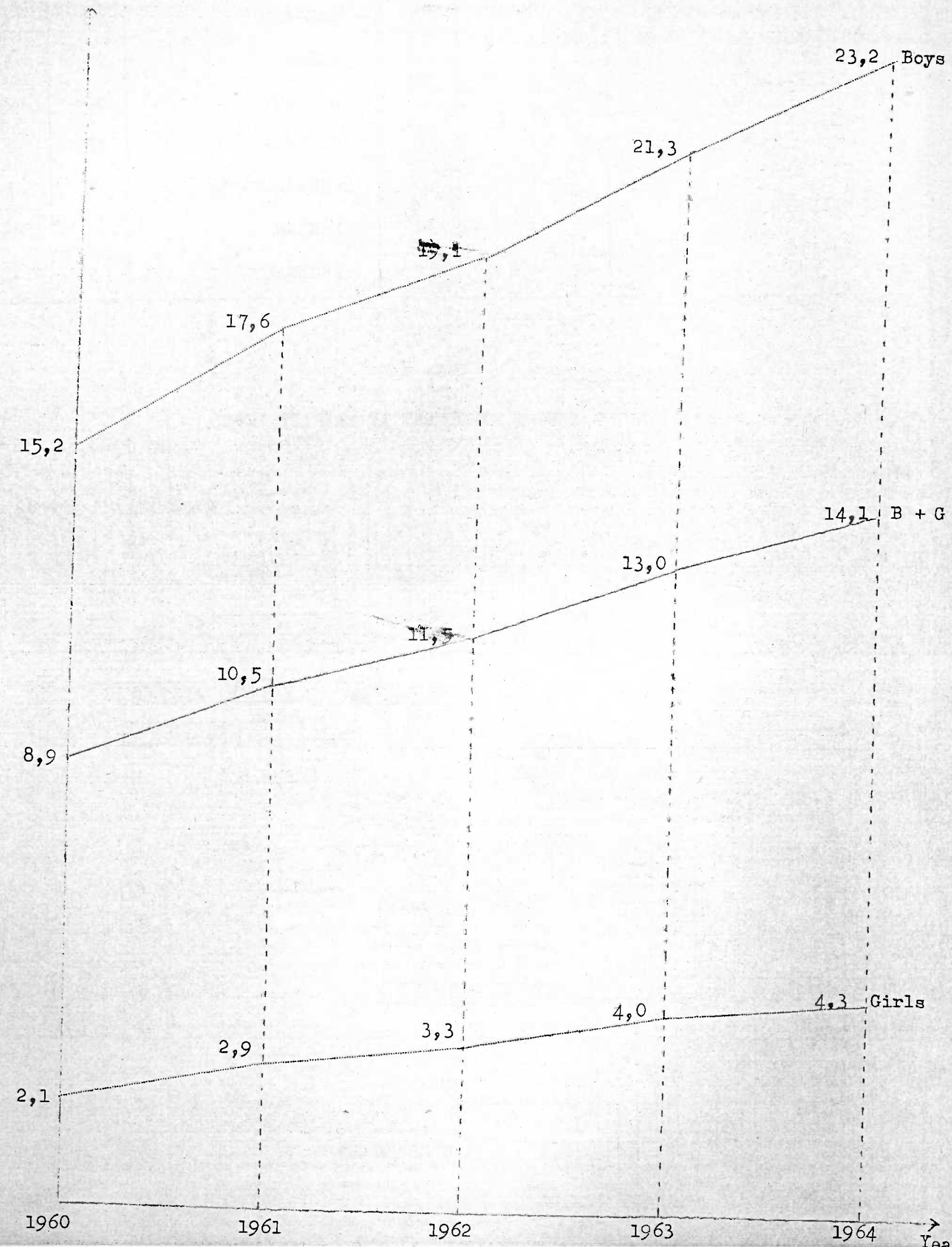


Table 20

Rates by provinces (1964)

\begin{tabular}{|c|c|c|c|c|c|c|c|}
\hline PROVIIVES & Boys & Girls & Total & PROVINCES & Boys & Girls & Total \\
\hline $\begin{array}{l}\text { KABUL - City and pro- } \\
\text { vince }\end{array}$ & 43 & 16 & 30 & GHAZINI & 29 & 2 & 16 \\
\hline FARAH & 53 & 6 & (31) & I.ATAGHAIV -TALOKAN & 27 & 4 & 16 \\
\hline BADAKHSHAII & 44 & 5 & 25 & NAINGARHAR & 28 & 3 & 16 \\
\hline IAZAR-I-SHARIF & $3 \varepsilon$ & 8 & 24 & $\begin{array}{l}\text { HERAT } \\
\text { GHORAT }\end{array}$ & 21 & & 13 \\
\hline DuAILANA & 34 & 11 & 23 & KAINDAFAR & 20 & 2 & 11 \\
\hline GRISHK & 40 & 1,5 & 22 & 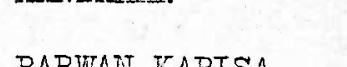 & & & \\
\hline $\begin{array}{l}\text { SHI BARGHAIT } \\
\text { PAKTIA }\end{array}$ & 36 & 0,3 & 18 & $\begin{array}{l}\text { BANIAIN } \\
\text { OROZGAIN }\end{array}$ & $\frac{10}{9}$ & $\begin{array}{l}3 \\
2 \\
0,4\end{array}$ & 7 \\
\hline
\end{tabular}

Fig. 7

IAP SHOWIIG SCHOOL MIROLITHT AT PRIEART IEVEL (1964)

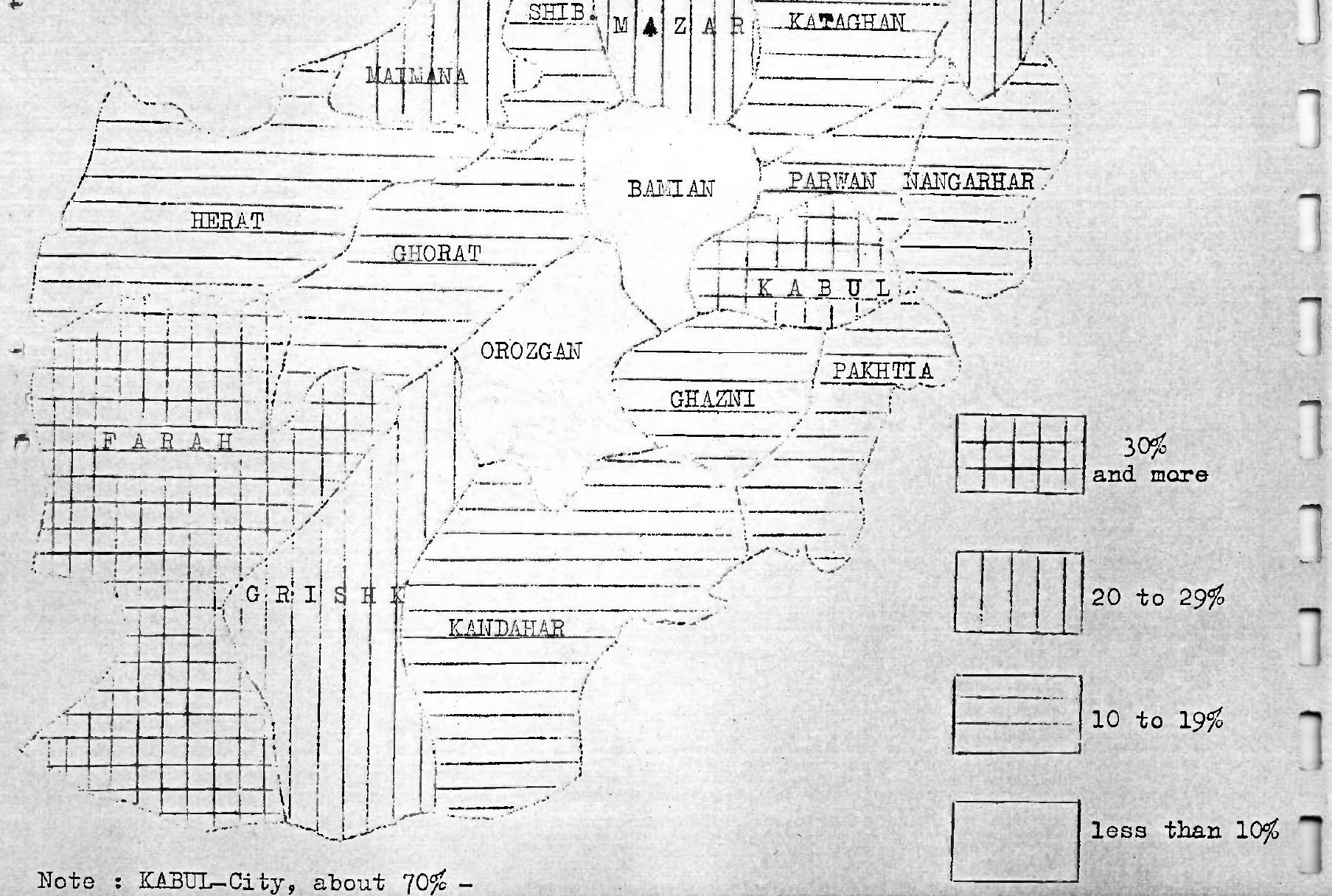


4 - Distribution of pupils according to the principal teaching language (1964)

Table 21

\begin{tabular}{|c|c|c|c|c|c|c|c|c|}
\hline & \multicolumn{3}{|c|}{ (1) PASHTO } & \multicolumn{3}{|c|}{ (2) $D \wedge R I$} & & \\
\hline & Boys & Girls & Total & Boys & Girls & Total & (I) & (2) \\
\hline $\begin{array}{c}\text { Village schools } \\
\text { (I to III) }\end{array}$ & 25811 & 1841 & 27,652 & 35276 & 6223 & 41499 & 40 & 60 \\
\hline $\begin{array}{c}\text { Primary schools } \\
(I \text { to VI) }\end{array}$ & 93656 & 3846 & 97502 & 108750 & 32758 & 141508 & 41 & 59 \\
\hline Total & 119467 & 5687 & 125154 & 144026 & 38981 & 183007 & & \\
\hline $\begin{array}{r}\text { Middle schools } \\
\text { (VII to IX) }\end{array}$ & 5372 & 182 & 5554 & 12303 & 3967 & 16270 & & \\
\hline $\begin{array}{c}\text { Iycees } \\
(\mathrm{X} \text { to XII }) \\
\end{array}$ & 720 & 47 & 767 & 2738 & 1023 & 3761 & & \\
\hline
\end{tabular}

(Vocational schools : Data not available)

Table 22

Distribution by provinces, at primary level (percentages)

\begin{tabular}{|c|c|c|c|c|c|}
\hline PROVINCES & PASHTO & DARI & PROVINCES & PASHTO & DARI \\
\hline KABUL - CitJ & 5 & 95 & KATAGHAIV & 22 & 78 \\
\hline L:BUL - Province & 56 & 44 & TALOIANT & 6 & 94 \\
\hline FARAH & 31 & 69 & INANGARHAR & $(99,9$ & 0,1 \\
\hline BADATHSHAIN & 8 & 92 & HERAT & 9 & 91 \\
\hline WAZAR-I-SHARIF & 25 & 85 & GHORAT & 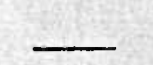 & (100) \\
\hline MAIULNA & 19 & 81 & KAINDAHAR & (100) & $\longrightarrow$ \\
\hline GRISHK & 100 & - & PARWANT & 2 & 98 \\
\hline SHI BARG AIV & 19 & 81 & KAPISA & 26 & 74 \\
\hline PAKTIA & 100 & - & BAIIIAN & - & 100 \\
\hline GHAZINI & (74) & 26 & OROZGAIJ & (73) & 27 \\
\hline
\end{tabular}


5 - Education of girls

Fig. 8

MAP SHONING SCHOOL ENROLLEENT OF GIRLS

AT PRIVIARY LEVEI (1964)

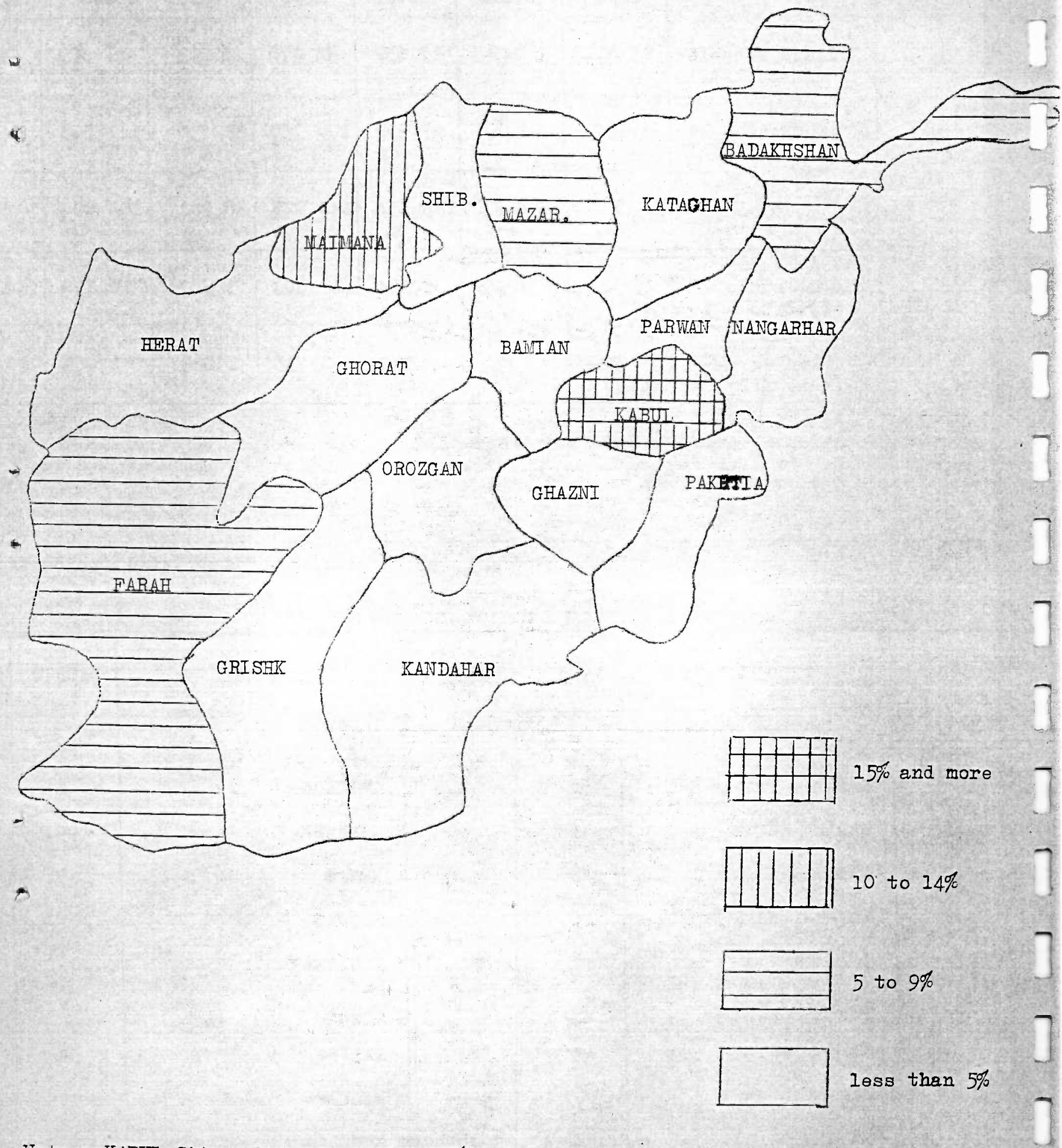

Note : KABUI-City : between 40 and 50\% 
Fig. 9

Girls-Boys Ratios between 1953 and 1964

Boys-Girls

(Primary level)

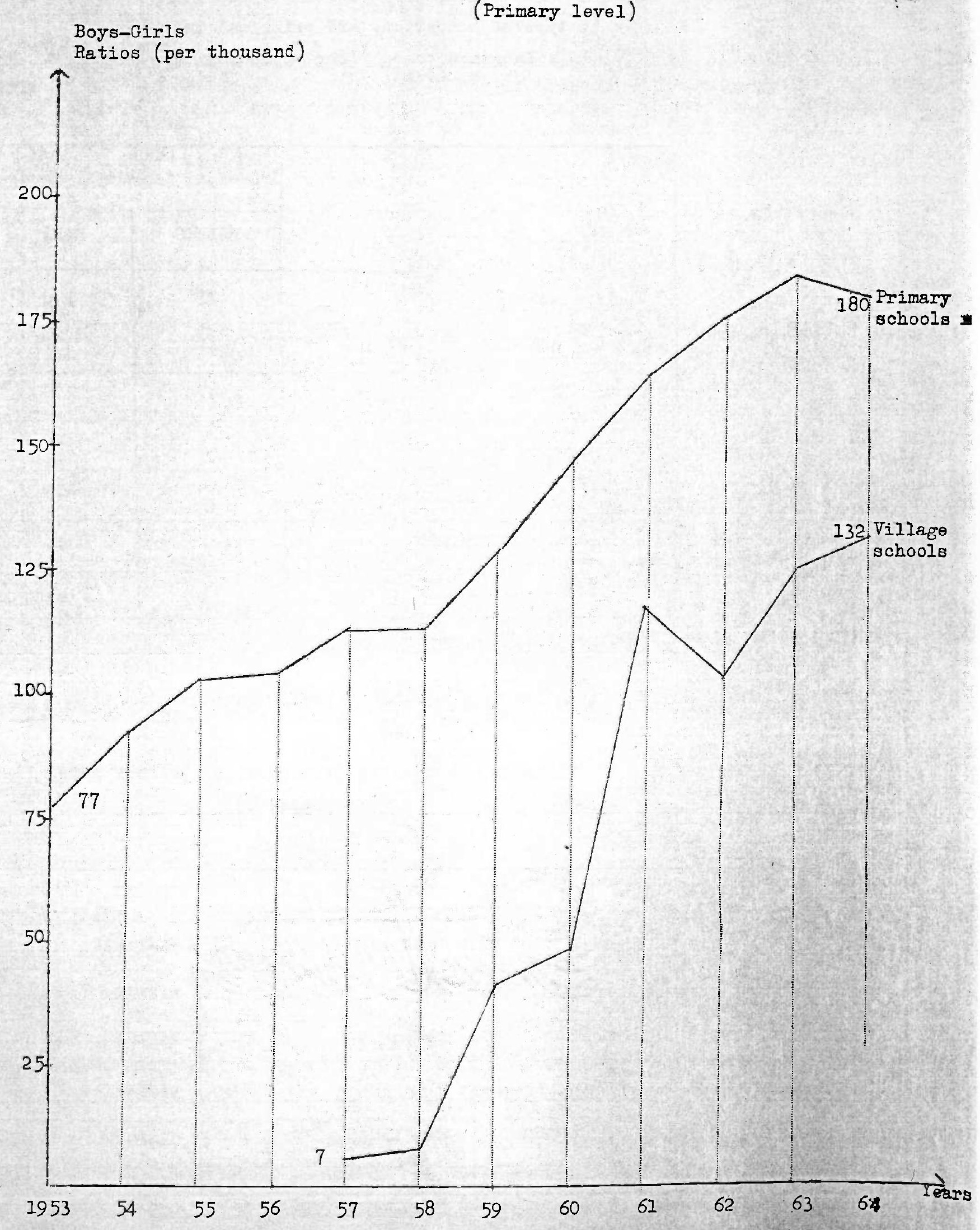

\# incl. primary classes attached to secondary and vocational schools 
Table 23

\section{Girls-Boys ratios (1964)}

by type of education, and principal teaching langrage used (per thousand)

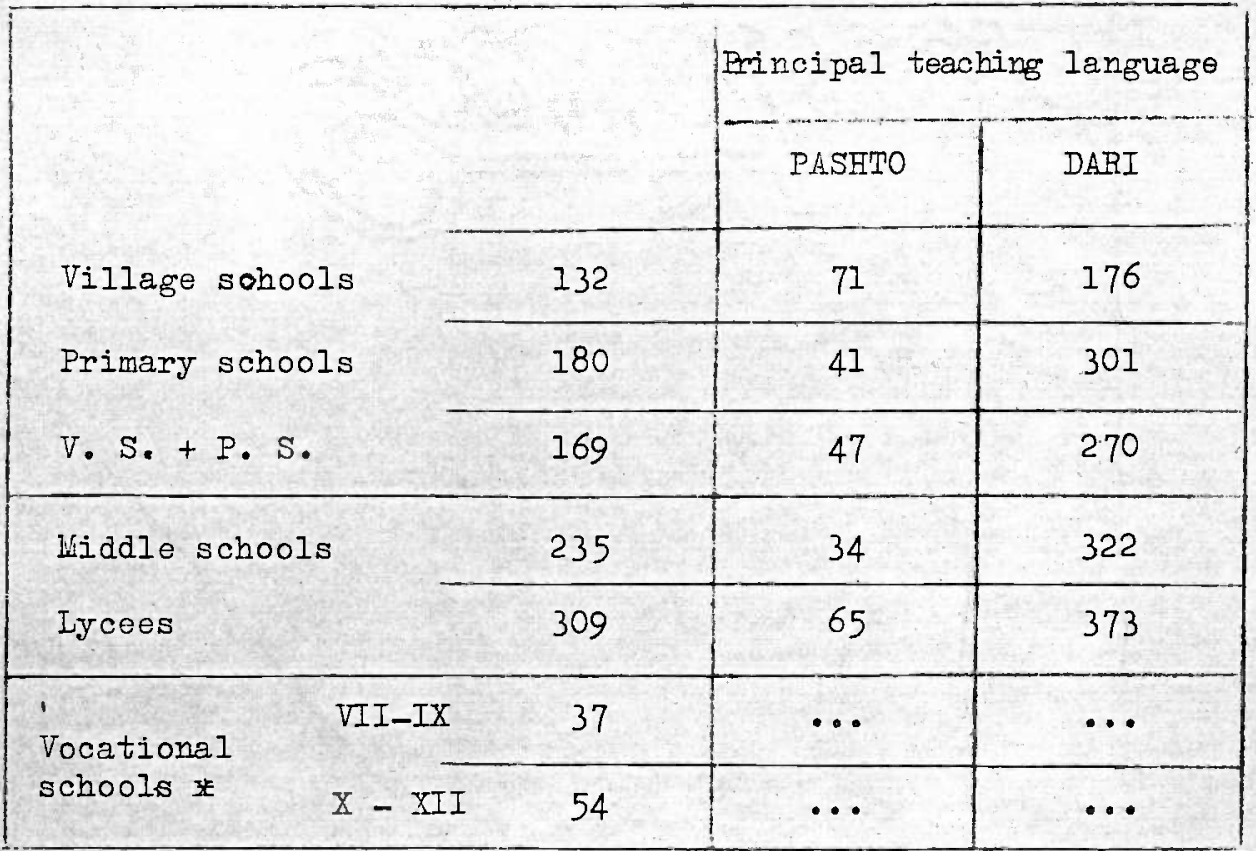

* incl. teacher training schools

Table 24

Girls-Boys ratios by provinces, at primary level (1964) (per thousand)

\begin{tabular}{|c|c|c|c|c|c|}
\hline KAPUL-City & 534 & SHI BARGHAIN & 123 & GHORAT & 84 \\
\hline KABUL-Province & 8.7 & PAKHIIA & 8 & KAINDAHAR & 96 \\
\hline FARAH & 102 & GHAZINI & 74 & PARTAIN & 130 \\
\hline BADALHSHAN & 102 & KATAGHAN & 192 & KAPISA & 164 \\
\hline MAZAR-I_SHARIF & 193 & TALOKAN & 95 & BAMIAN & 133 \\
\hline RAIUANA & 293 & NAIGGARHAR & 105 & OROZGAIT & 44 \\
\hline GRISHK & 35 & HERAT & 175 & AVERAGE & 169 \\
\hline
\end{tabular}


b) Secondary schools

Table 27

Mean ages by grade (Kabul-City - 1964)

\begin{tabular}{|c|c|c|c|c|c|c|}
\hline GRADI & VII & VIII & IX & X & XI & XII \\
\hline Normal age & 13 & 14 & 15 & 16 & 17 & 18 \\
\hline Moys & 15,2 & 16,4 & 17,1 & 19,2 & 19,9 & 21,2 \\
\hline Age Girls & 14,0 & 15,4 & 16,6 & 18,0 & 18,6 & 19,8 \\
\hline
\end{tabular}

Table 28

Percentage of pupils who are two or mikre years older than the numil age for each grade ( Kabil - City - 1964)

\begin{tabular}{|c|c|c|c|c|c|c|}
\hline & VII & VIII & IX & $X$ & XI & XII \\
\cline { 2 - 6 } Boys & $63 \%$ & 70 & 62,5 & 77,6 & $6 I, 5$ & 50,5 \\
\hline Girls & $26.1 \%$ & 38,9 & 47,9 & $56, I$ & 48,6 & 57,6 \\
\hline
\end{tabular}


c) Vocational schools

Table 29

Mean ages by grade (KabuI - City - 1964)

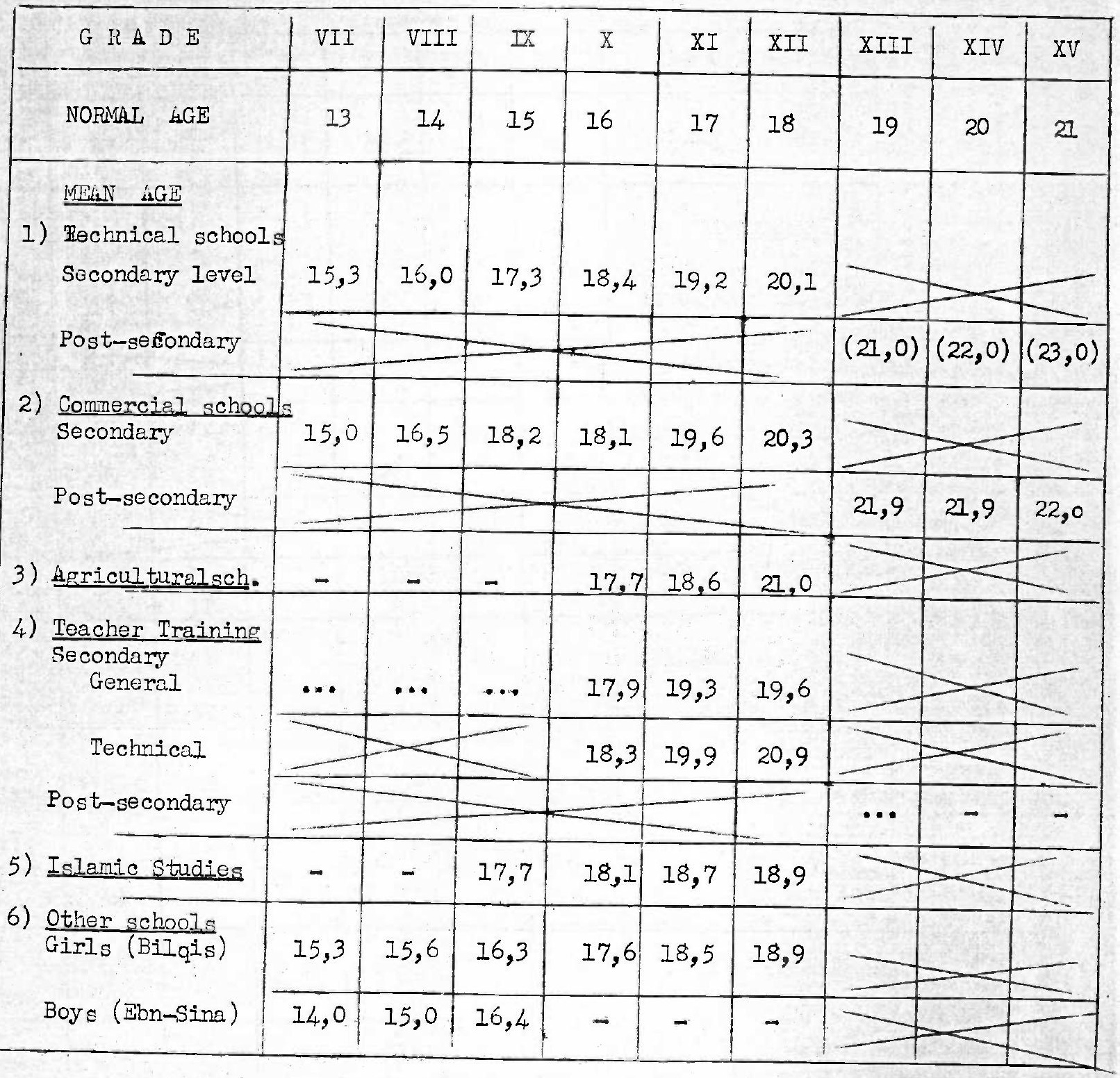

(I) Provincial pupils boarding in Kabul schools are on the average older than Kabul pupils. 
Table 30

Percentage of pupils who are two or more years older than the normal age for each grade

(KLBUL - CITY - 1964)

\begin{tabular}{|c|c|c|c|c|c|c|c|c|c|}
\hline$G R \& D E$ & VII & VIII & $I X$ & $\mathrm{x}$ & $\mathrm{XI}$ & $X I I$ & XIII & XIV & $\mathrm{XV}$ \\
\hline MORMLIL IIGE & 13 & 14 & 15 & 16 & 17 & 18 & 19 & 20 & 21 \\
\hline $\begin{array}{l}\text { PUECENTiGE } \\
\text { 1) Technical schools } \\
\text { Secondary level }\end{array}$ & 70 & 63 & 85 & 75 & 73 & 92 & & & \\
\hline Post-secondary & & & $=$ & 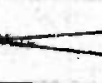 & -7 & E & $(100)$ & $(100)$ & (100) \\
\hline 2) Commercial school & & & & & & & & & \\
\hline Secondarge & 60 & 73 & 84 & 65 & 72 & 78 & & & \\
\hline Post-secondary & & Z & $=$ & $c$ & C & & 100 & 73 & $(50)$ \\
\hline 3) isricultural sch. & - & - & - & 70 & 46 & 85 & & & \\
\hline 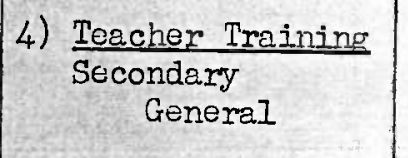 & $\cdots$ & $\cdots$ & .. & 85 & 52,5 & 57 & & & \\
\hline Technisal & 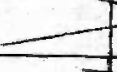 & & 3 & 71 & 88 & 100 & & & \\
\hline Post-secondary & & & $=$ & $\leftarrow$ & & & & - & - \\
\hline 5) Islamic Studies & - & - & 73,5 & 58 & 56,5 & 40 & & & \\
\hline 6) $\frac{\text { Other schools }}{\text { Girls (Bilqis) }}$ & 89 & 50 & 43 & 73 & 61 & 14 & & & \\
\hline Boys (Ebn-Sina) & 35 & 39 & 44 & - & - & - & & & \\
\hline
\end{tabular}


Fig. 12

Cohortsbeginning in 1959, by sex

a) Boys

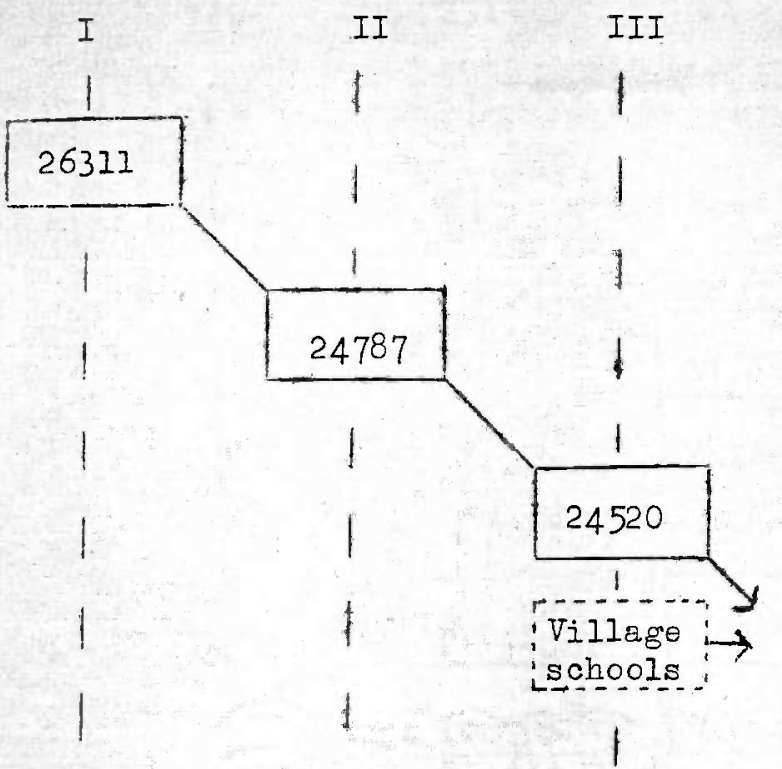

b) Girls

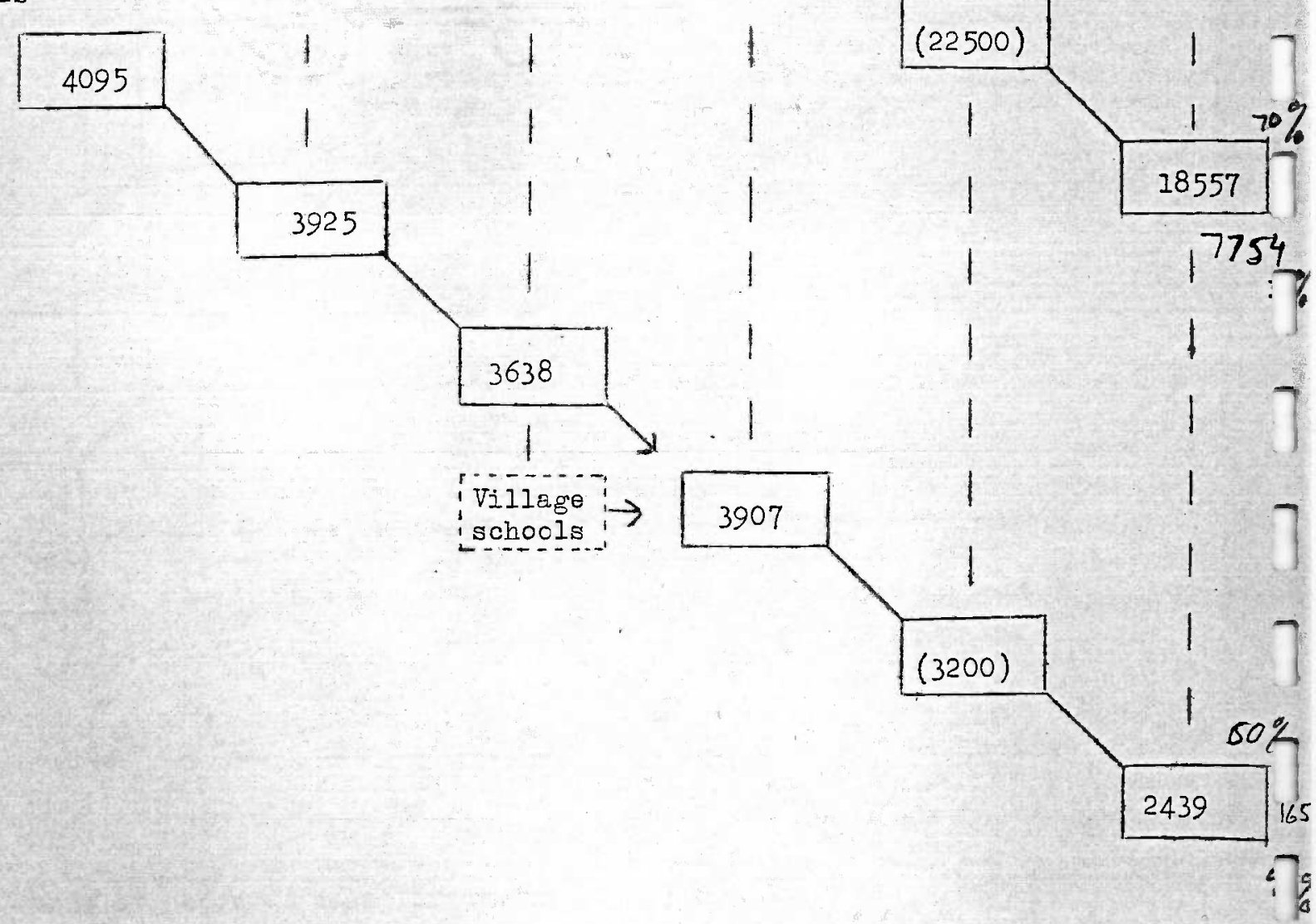




\section{Estimated promotion, repeater and drop-out rates (1963-1964)}

Fig. 13

(in percentages of 1963 enrolment)
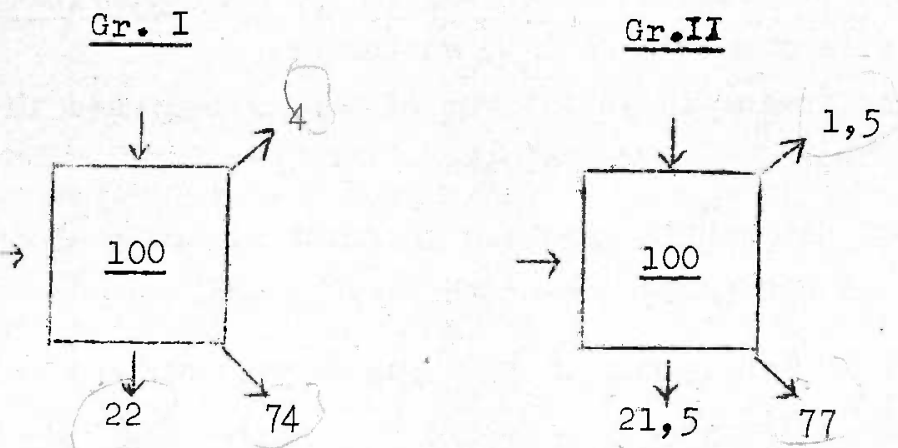

$\underline{\text { Gr.III }}$

$\underline{\text { Gr.IV }}$

$\underline{\mathrm{Gr} . \mathrm{V}}$
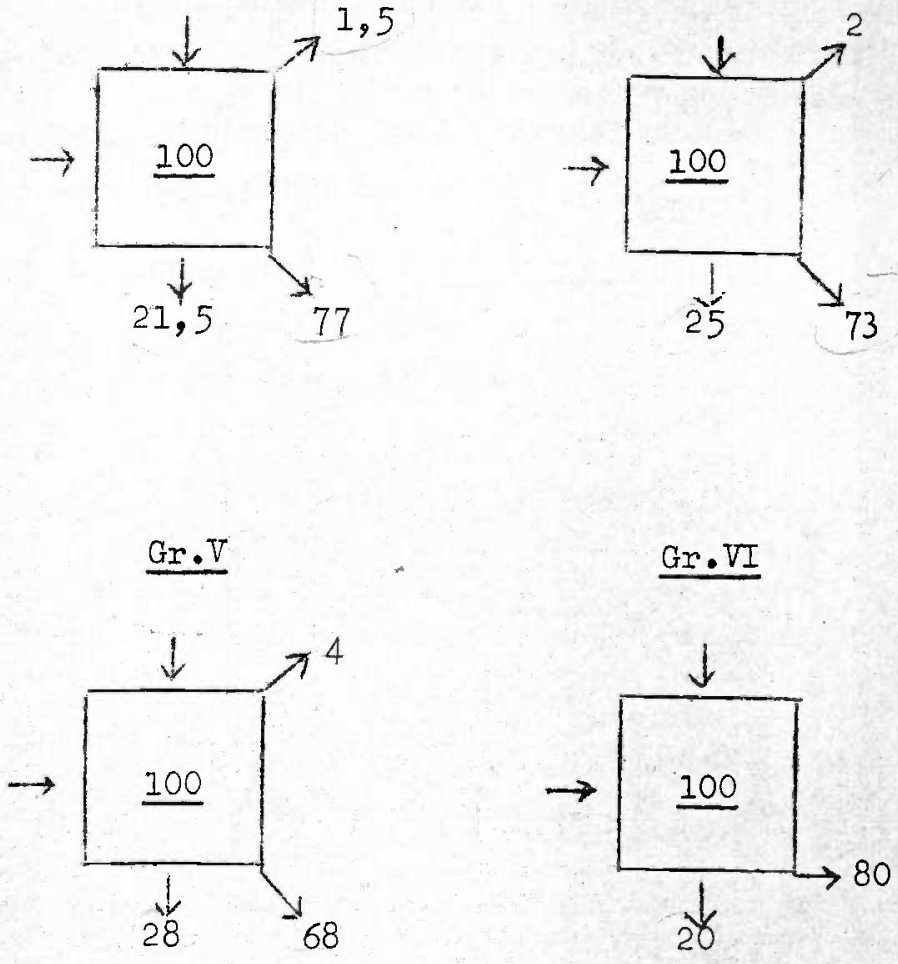

Repeaters ( $r$ )

(in the same grade during the precedent year)

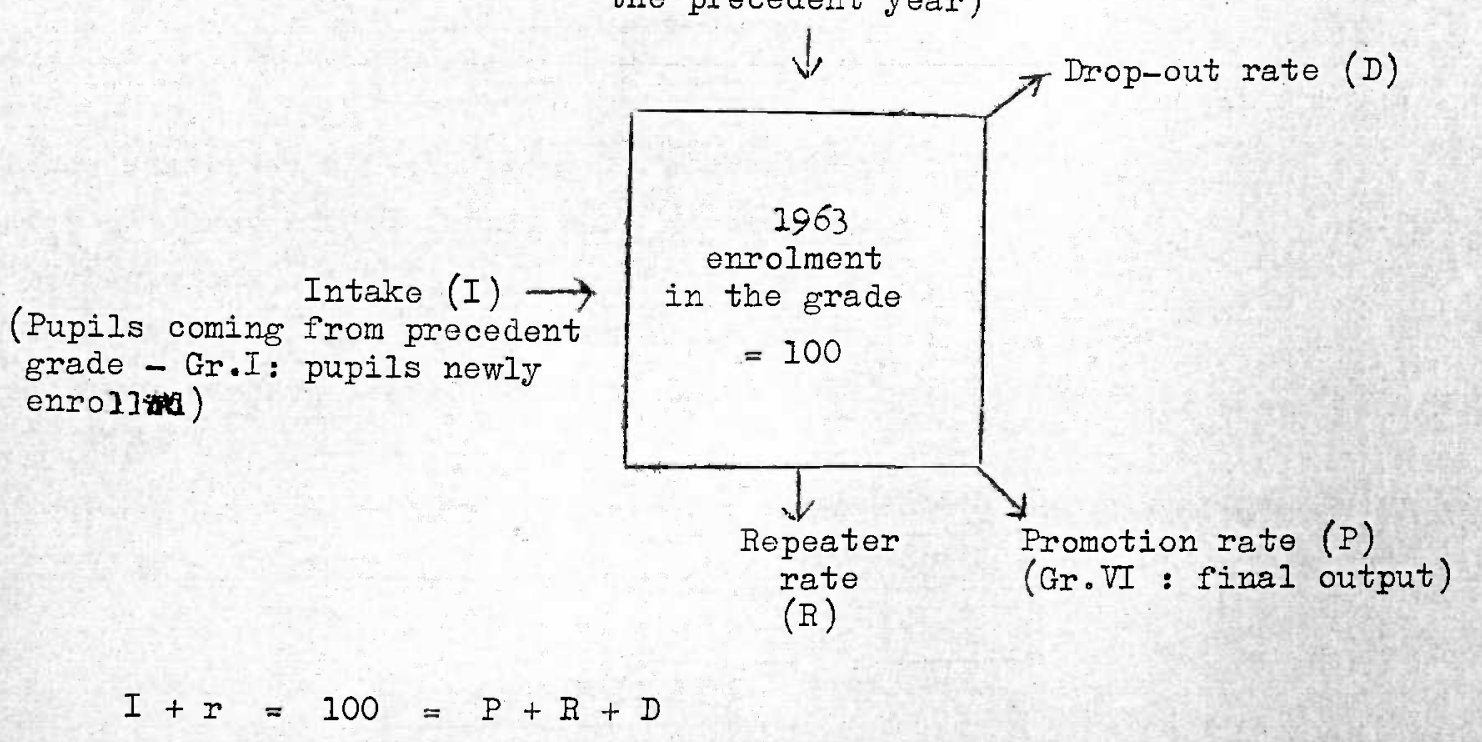

Fig. 13 gives the promotion, repeater and drop-out rates estimated for each primary school grade (village schools excluded). 
With this set of calculations worked out to its culmination at grade. VI, tho total number of years of primary schooling incurred for this initial group of 1000 pupils amounts to 6720 . On the other hand, those who would in fact complete grade VI on these calculations would amount to 783 , which on the basis of a 6 -year cycle, at iceal efficiency, should require only $6 \times 783=4698$ years. The coefficient of present efficicncy is therefore :

$$
\frac{4698}{6720}=0,69(0,70 \text { in round figures })
$$

The system is therefore working at a level of only $70 \%$ of ideal "efficiency" as cefined abave. 
Fig. 14

Theoretical school life of a group of 1000 pupils newly enrolled in grade I

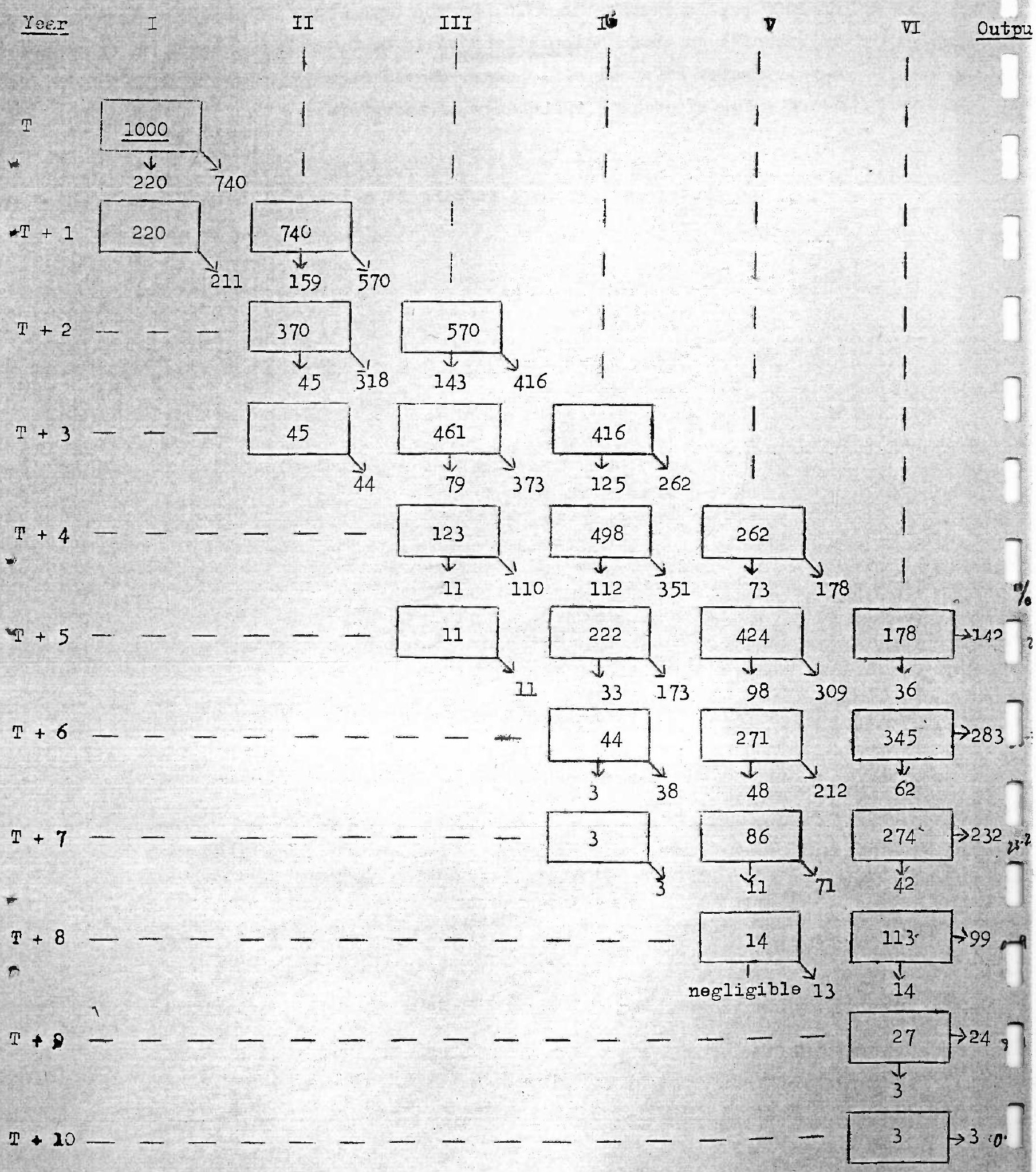

Total number of school-years spent $=6720$ Average per pupil $=8,58$ years $(6720 / 783)$ 
8. Wastace at secondarv level

a.) Retention rates

It is necessary to observe that the retention rates fust not be confounded with the promotion rate.

In fact, a school cohort is different from a demographtc cohort: because of the numizer of repeaters it is not exactly the same individuals who are found in the successive grades from one year to another.

The retention rates are the result of the rate of promotion, the number of drop-outs and of repeaters, factors which ought to be accurately dofined, if the school system is to be fully evaluated.

Figure 15

Retention rates in secondary schools

(iiddle schools and lycees)

- Boys and girls -
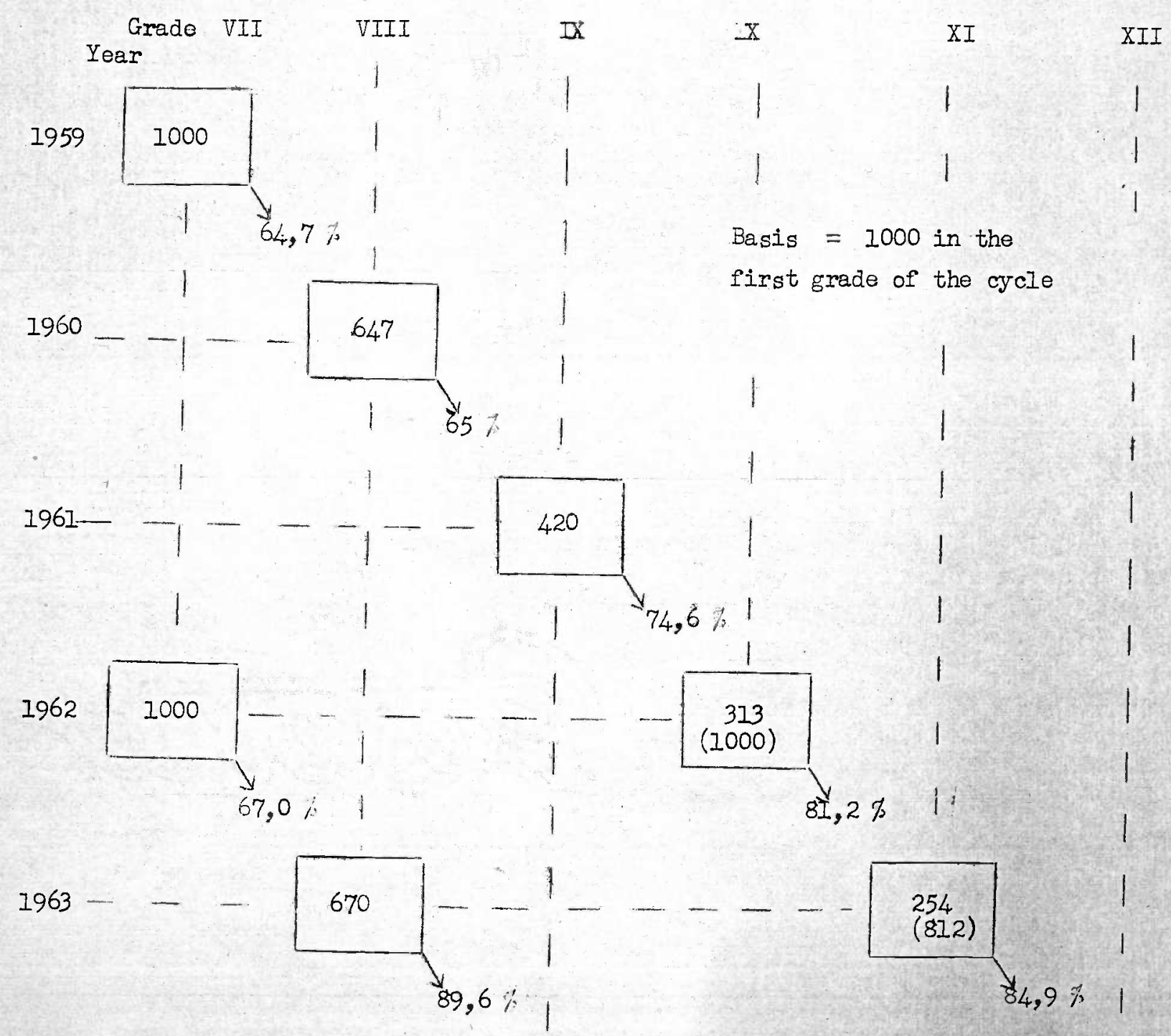
Retention rates in secondary schools

(viiddle schools and lycees)

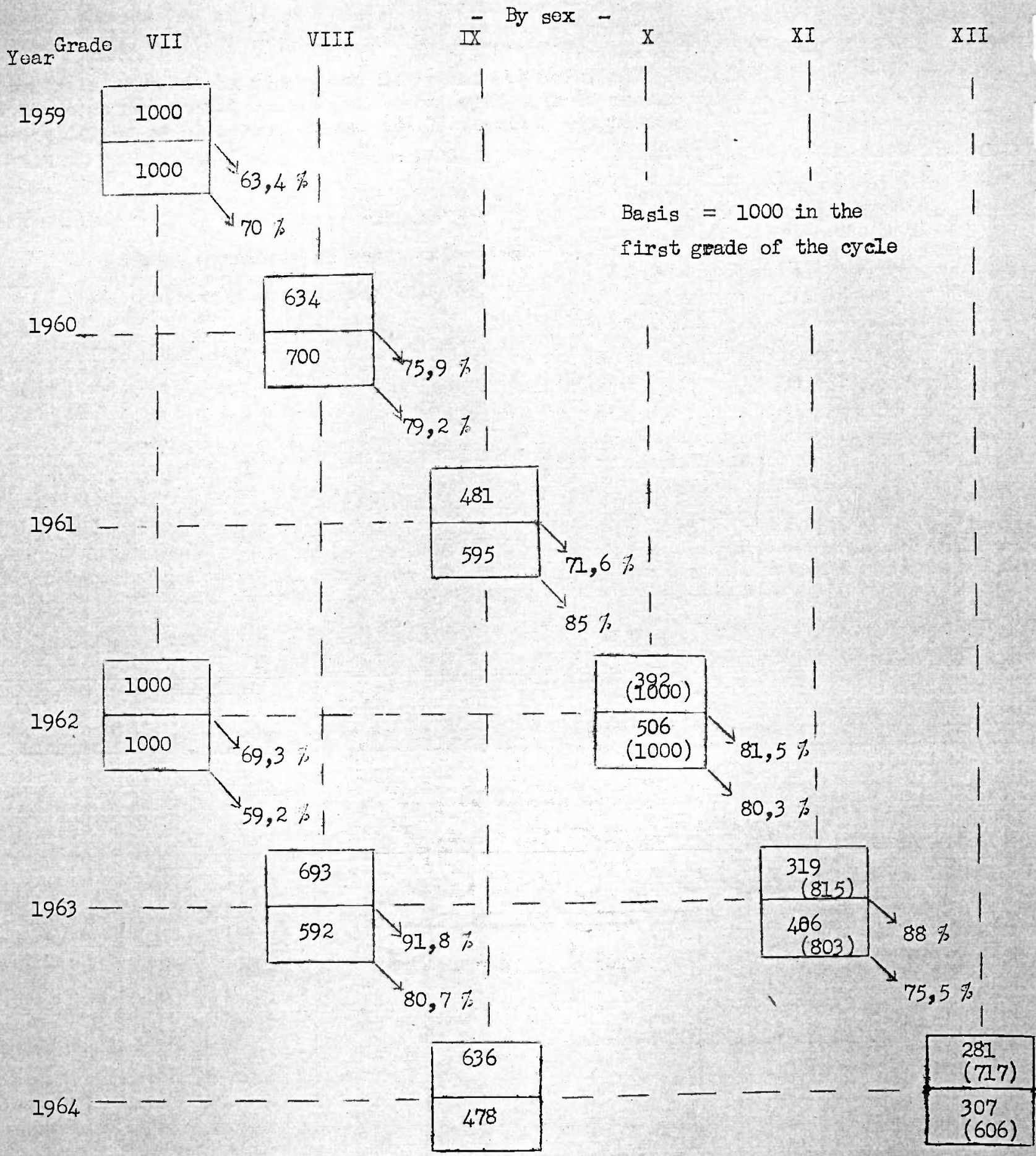


Figure 17

Retention rates in technical schools

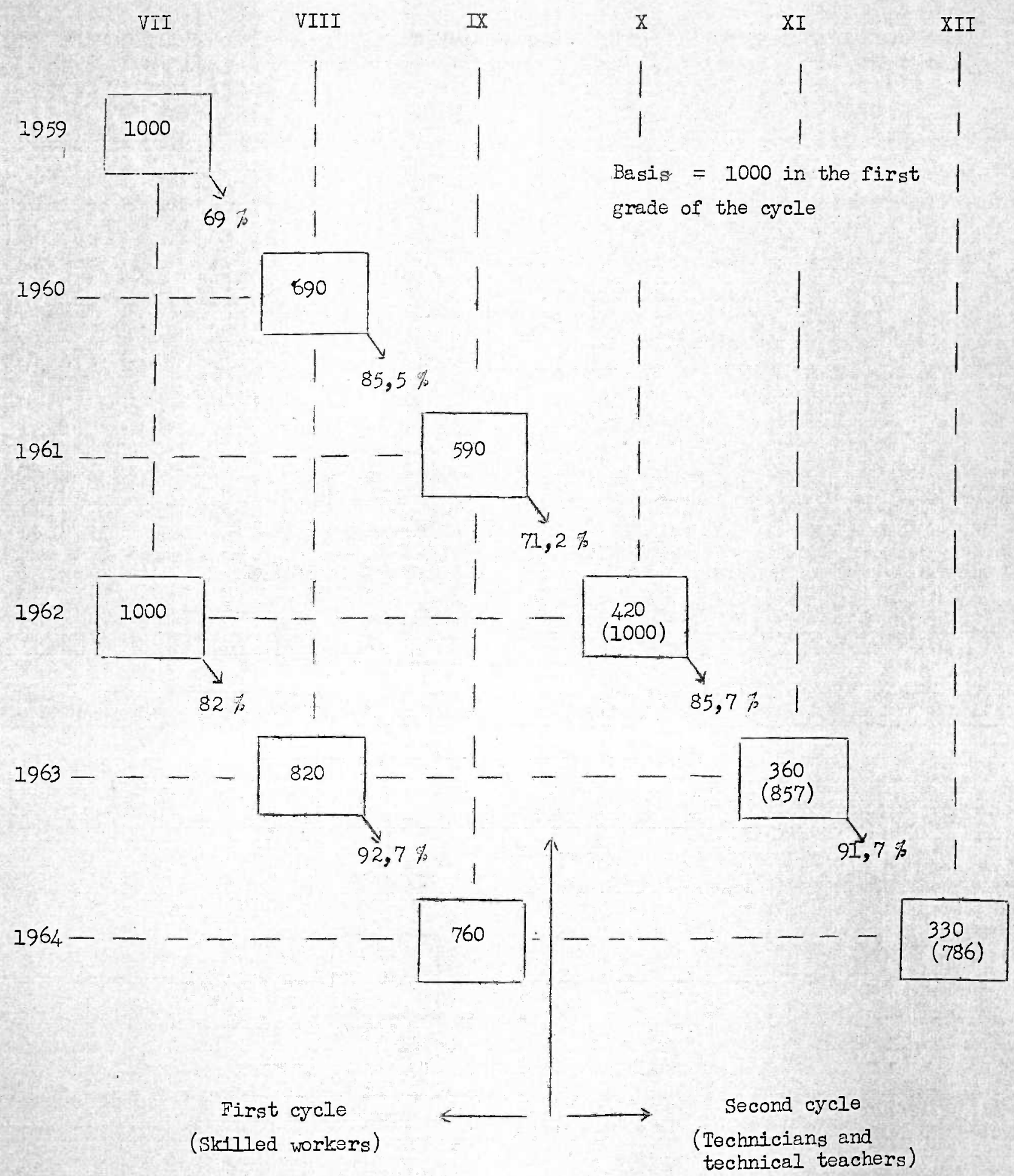




\section{Figure 18}

Retention rates in commercial schools

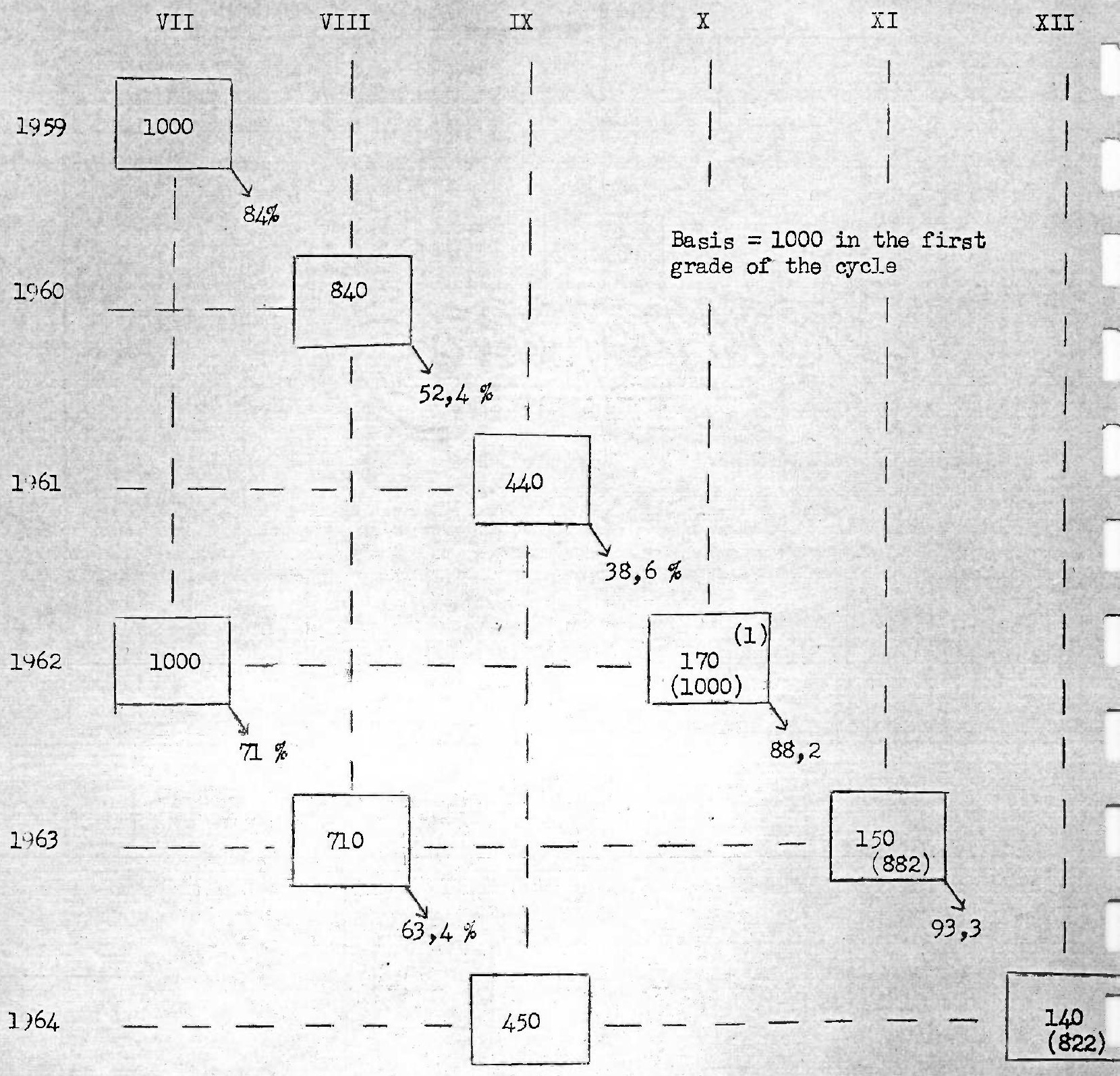

(1) With pupils coming from Middle schools 
Figure 19

Retention rates in igricultural Schools

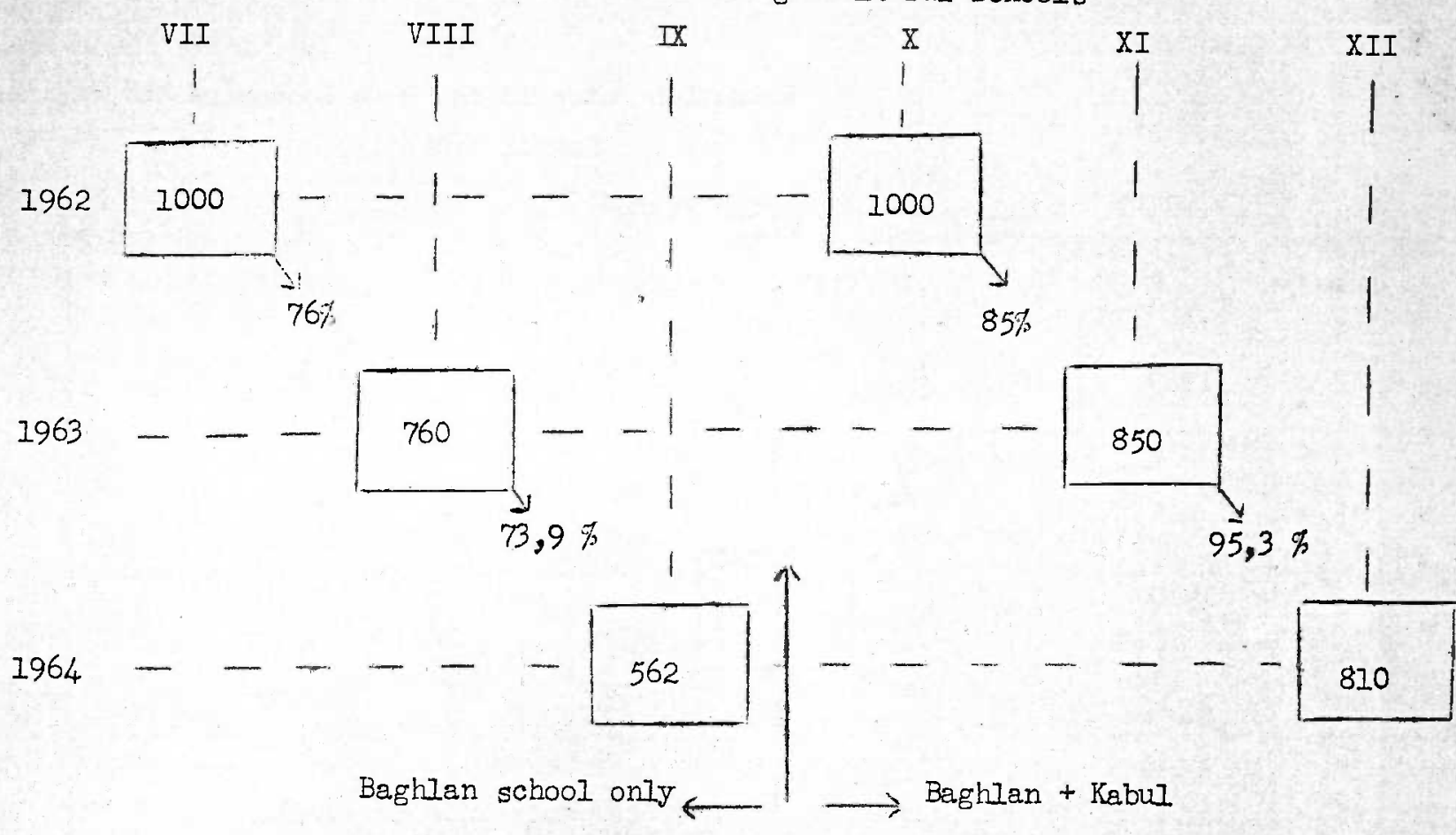

(Basis $=1000$ in the first grade of the cycle)

Figure 20

Retention Rates in Teacher Training Schools

X

XI XII:

$1962 \quad \begin{array}{lll}1000 & 1 & 1 \\ 81,6 \% & 1 & 1\end{array}$

$1963 \ldots-\left.\sqrt{816}\right|_{99,0 \%}$

$1964----\sqrt{808}$

Note: The se figures are ba sed on the total figures for Kabul and Jalalabad. Herat did not operate as a DMl in 1962 
Figure 27

Retention rates in the $\mathrm{H}$, ie Economics and Business School (Bilq1s)

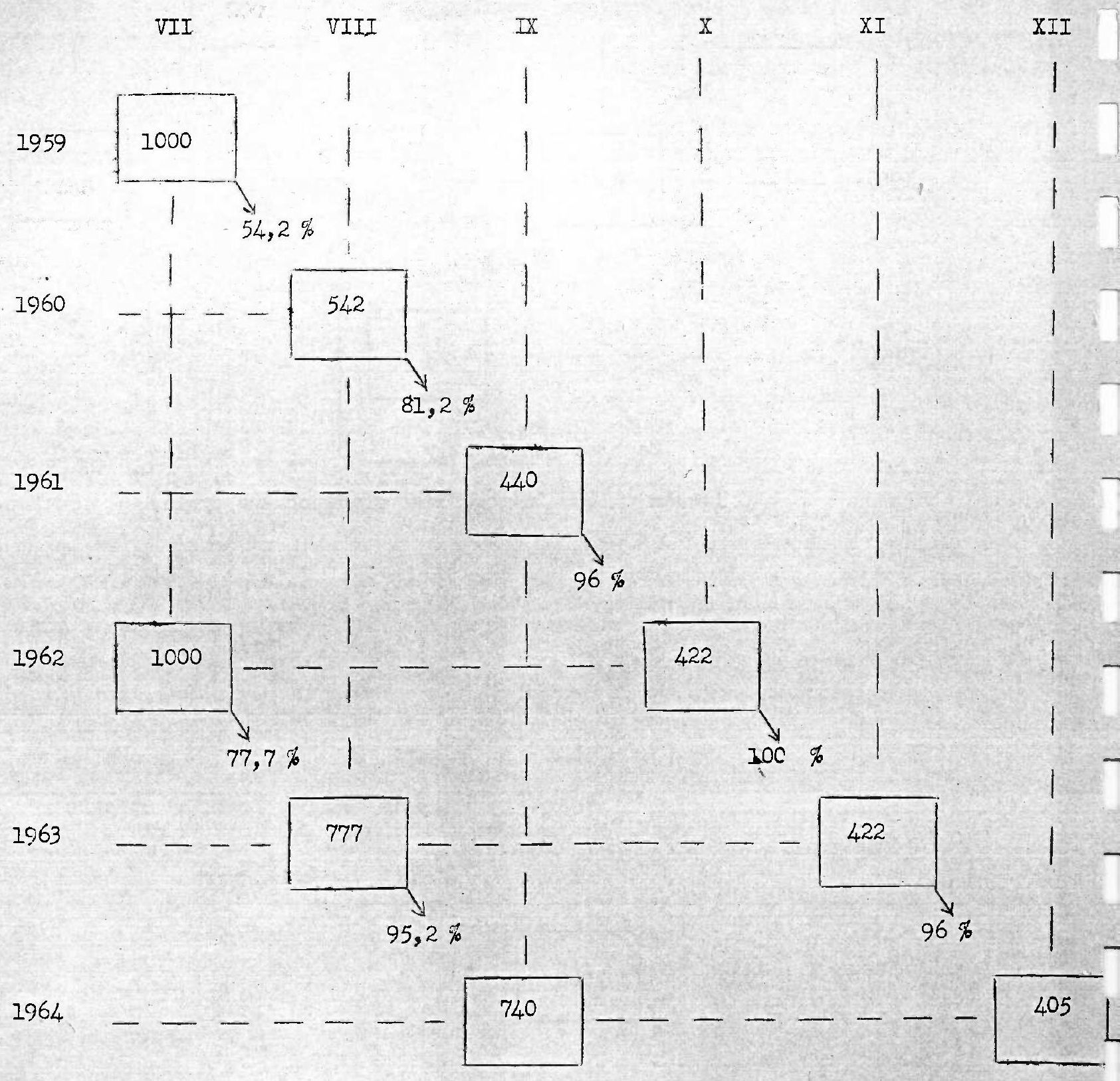

Basis $=1000$ in grade VII 
Figure 25

Promotion, repeater and drop-out rates

in the Kabul Agriculture School (1963 $\rightarrow 1964)$

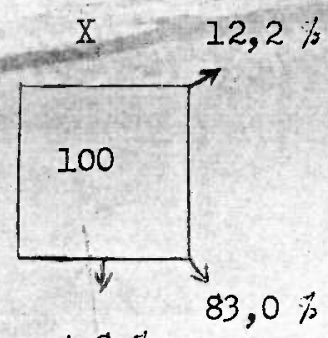

$4,8 \%$

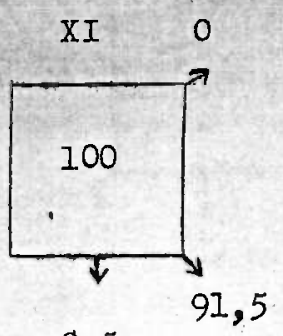

8,5
XII

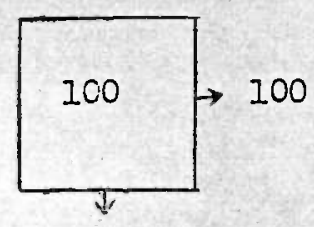

0

Figure 26

Promotion, repeater and drop-out rates

in the Home Evonomics and Business School (Bilqis)

$$
(1963 \rightarrow 1964)
$$
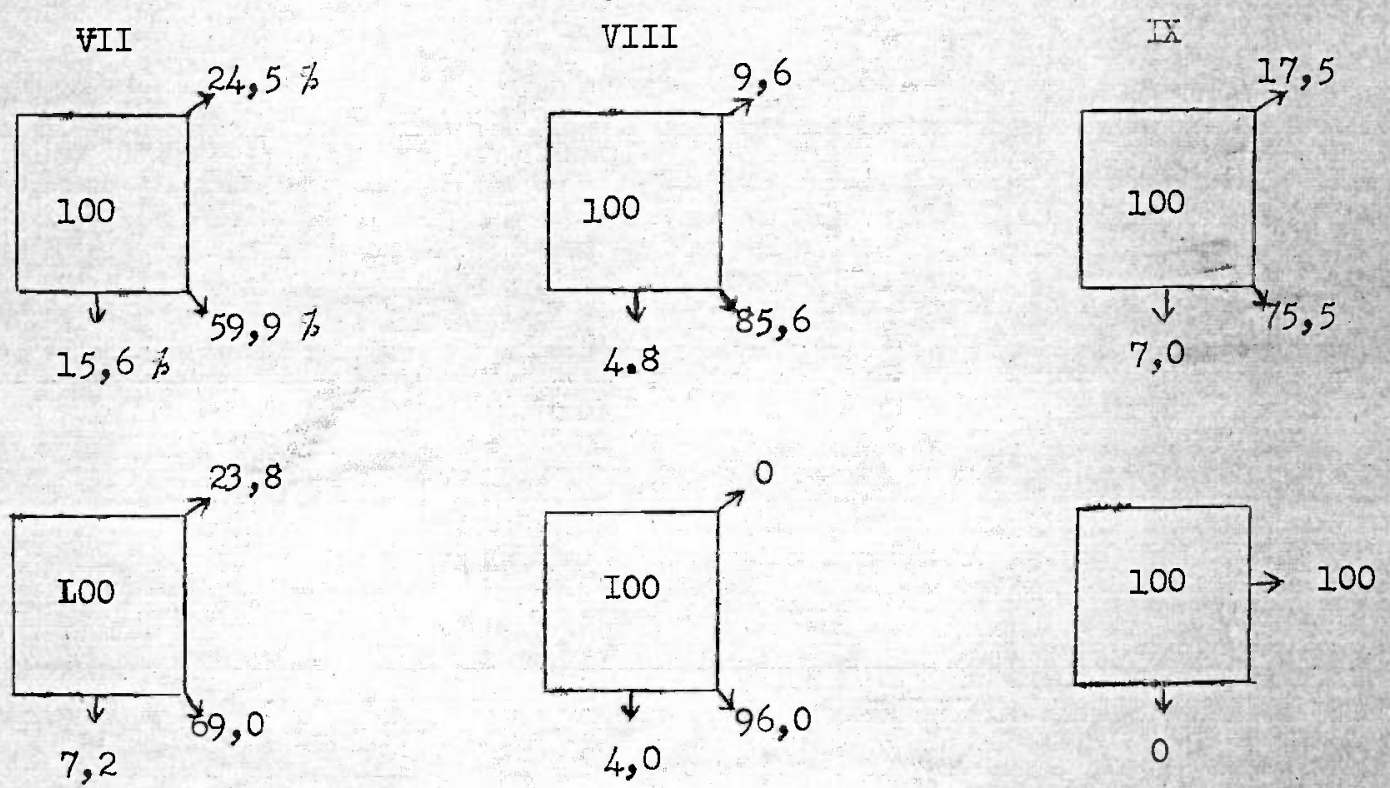

Figure 27

Promotion, repeater and drop-out rates in the Technical Tdacher Training College (1963 $\rightarrow$ 1964)

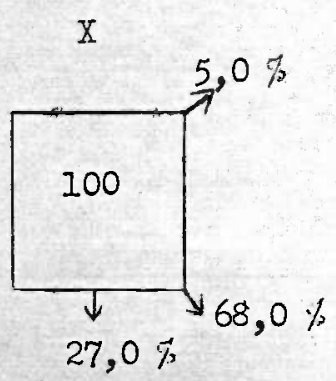

XI

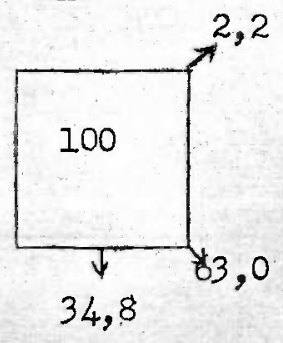

XII

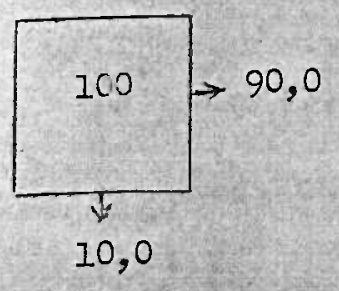


9. Gradustes (Primary and secondary levels)

Table 31

Grade VI $(1960$ - 1963)

\begin{tabular}{|c|c|c|c|c|c|c|c|c|c|}
\hline \multirow[b]{2}{*}{ Yen:r } & \multicolumn{3}{|c|}{ BOYS } & \multicolumn{3}{|c|}{ GIRLS } & \multicolumn{3}{|c|}{ TOTAL } \\
\hline & $\begin{array}{l}\text { Enrol. } \\
\text { gr. VI }\end{array}$ & Gradur & $\%$ & Gr. VI & Gradua & $\begin{array}{l}\text { es } \\
\%\end{array}$ & Gr. VI & Gradu & $\begin{array}{l}\text { tes } \\
1 \%\end{array}$ \\
\hline 1960 & 10820 & ... & ... & 1167 & $\cdots$ & $\cdots$ & 11987 & 9590 & 80 \\
\hline 1961 & 12649 & $\cdots$ & ... & 1410 & $\cdots$ & ... & 14059 & 11247 & 80 \\
\hline 1962 & 14582 & 10574 & 72 & 1668 & 1293 & 77 & 16250 & 11867 & 73 \\
\hline 1963 & (16000) & 12333 & (77) & $(2000)$ & 1417 & (70) & $(18000)$ & 13750 & $(76)$ \\
\hline
\end{tabular}

(1) Including those who passed grom a grade VI to grade VII in primary classes attached to secondary and vocational schools.

Table 32

Secondary schppfs - Grade IX (1961 - 1963 3 )

\begin{tabular}{|c|c|c|c|c|c|c|c|c|c|}
\hline \multirow[b]{2}{*}{ Year } & \multicolumn{3}{|c|}{ BOYS } & \multicolumn{3}{|c|}{ GIRLS } & \multicolumn{3}{|c|}{ TOTAL } \\
\hline & $\begin{array}{l}\text { Enrol: } \\
\text { gr. IX }\end{array}$ & Gradu & $\begin{array}{l}\text { tes } \\
1 \%\end{array}$ & Gr. IX & Gradr & $\begin{array}{l}\text { es } \\
\%\end{array}$ & Gr. IX & Gradue & \\
\hline 1961 & $\ldots$ & $\cdots$ & $\cdots$ & $\cdots$ & $\cdots$ & $\ldots$ & $(2100)$ & 1796 & (35) \\
\hline 1962 & ... & 1595 & $\cdots$ & $\cdots$ & 298 & $\ldots$ & $(2350)$ & 1893 & (82) \\
\hline 1963 & 2791 & 2060 & 74 & 578 & 456 & 79 & 3369 & 2516 & 75 \\
\hline
\end{tabular}

(1) Independsint MiddIe Schools and M.s. attached to Lycees

(2) Includine those who passed from a grade IX to Grade X in Lycees

Table 33

Lycees - Grade XII (1961 - 1963)

\begin{tabular}{|c|c|c|c|c|c|c|c|c|c|}
\hline \multirow{2}{*}{ Year } & \multicolumn{3}{|c|}{ BOYS } & \multicolumn{3}{|c|}{ GIRL,S } & \multicolumn{3}{|c|}{ TOTAL } \\
\hline & $\begin{array}{l}\text { Enrol. } \\
\text { gr. XII }\end{array}$ & Gradu & & Gr.XII & Gradu & s & Gr. XII & Graduat & $\%$ \\
\hline 1961 & ... & $\cdots$ & $\cdots$ & $\cdots$ & $\cdots$ & $\cdots$ & (700) & 540 & $(77)$ \\
\hline 1962 & ... & 480 & ... & ... & 153 & ... & $(750)$ & 633 & (84) \\
\hline 1963 & 652 & 597 & 91 & 179 & 175 & 98 & 831 & 772 & $\$ 3$ \\
\hline
\end{tabular}


Table 34

Vocational schools - Grade IX (1961 - 1963)

Types of school

Table $\quad 35$

Vocational schools - Grade XII (1961 - 1963)

Types of school

Technical schools

Commercial schools

Agricultural schools

Islamic 'schools

Teacher Training

General

Technical

Sports

Bilqis school

\begin{tabular}{|c|c|c|c|c|c|c|c|c|}
\hline & & & & 962 & & & 963 & \\
\hline Gr.IX & Grac & $\begin{array}{c}\text { ates } \\
\%\end{array}$ & Gr. IX & Gradu & $t_{7}$ & Gr.IX & Grac & tes \\
\hline 262 & 248 & 95 & 275 & 255 & 93 & 295 & 288 & 97 \\
\hline 65 & 62 & 95 & 38 & 35 & 92 & 34 & 34 & 100 \\
\hline 325 & 254 & 78 & 451 & 325 & 72 & 353 & 275 & 78 \\
\hline 19 & 15 & 79 & 23 & $2 I$ & 91 & 28 & 19 & 68 \\
\hline
\end{tabular}


Table 36

Estimated Percentage of pupils passing from Grade VI to Grade VII $(1960-1963)$

\begin{tabular}{|c|c|c|}
\hline Year & $\begin{array}{c}\text { Enrolment in } \\
\text { Grade VI }\end{array}$ & $\begin{array}{c}\text { \% of pupiIs } \\
\text { passing } \\
\text { VII th Gr, }\end{array}$ \\
\hline 1960 & 11987 & $48 \%$ \\
1961 & 14059 & $50 \%$ \\
1962 & 16250 & $\ldots$ \\
1963 & $(18000)$ & $59 \%$ \\
\hline
\end{tabular}

Table 37

Destination of 1963 graduates - Grade IX

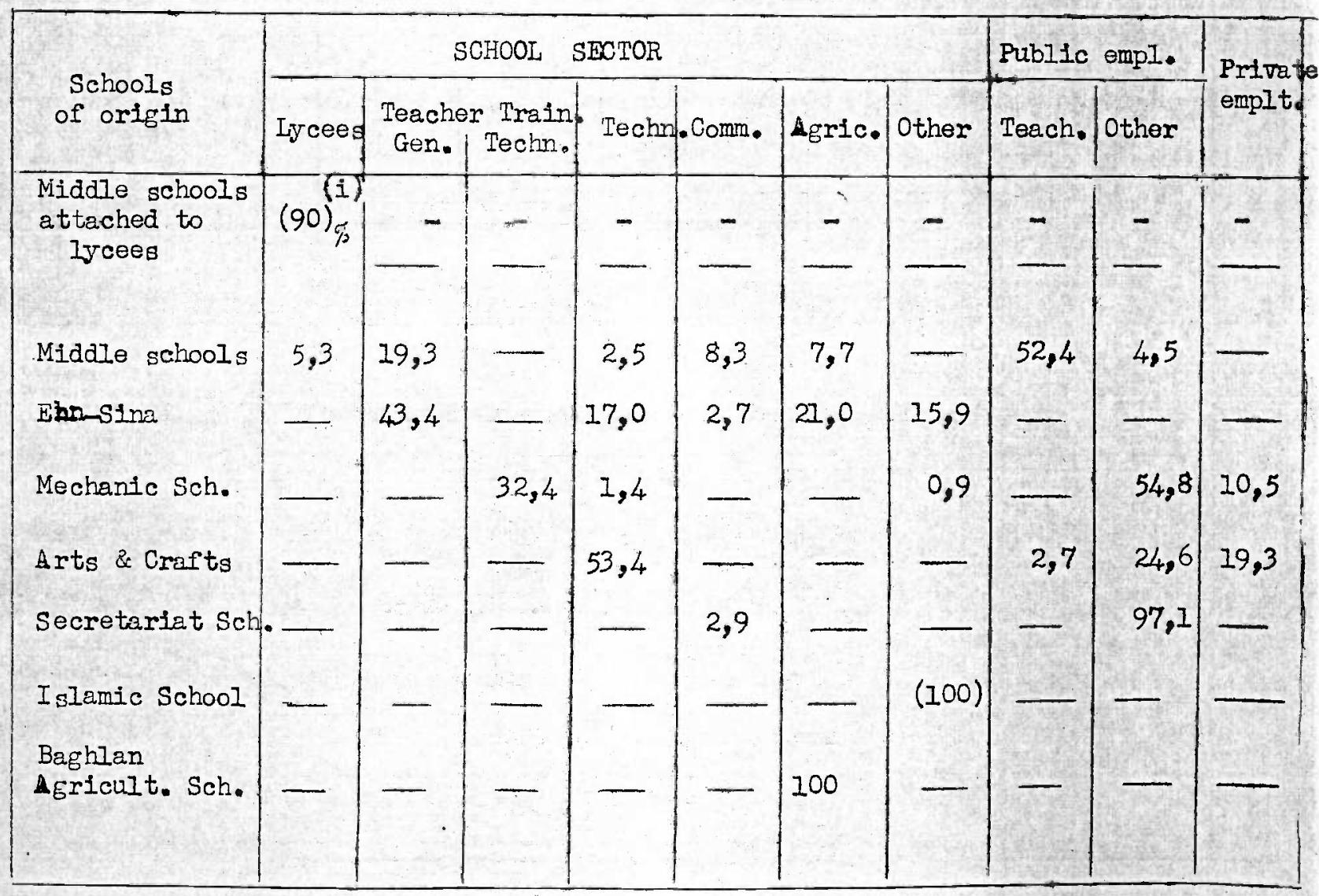

Source: Destination of students (Ministry of Education

Directorate of vocationel guidance - Ministry of Planning

Directorate of mampower - 1964)

(i) $10 \%$ drop-outs of the graduates estimated 
Table $\quad 38$

Destination of 1963 graduates - Grade XII (1)

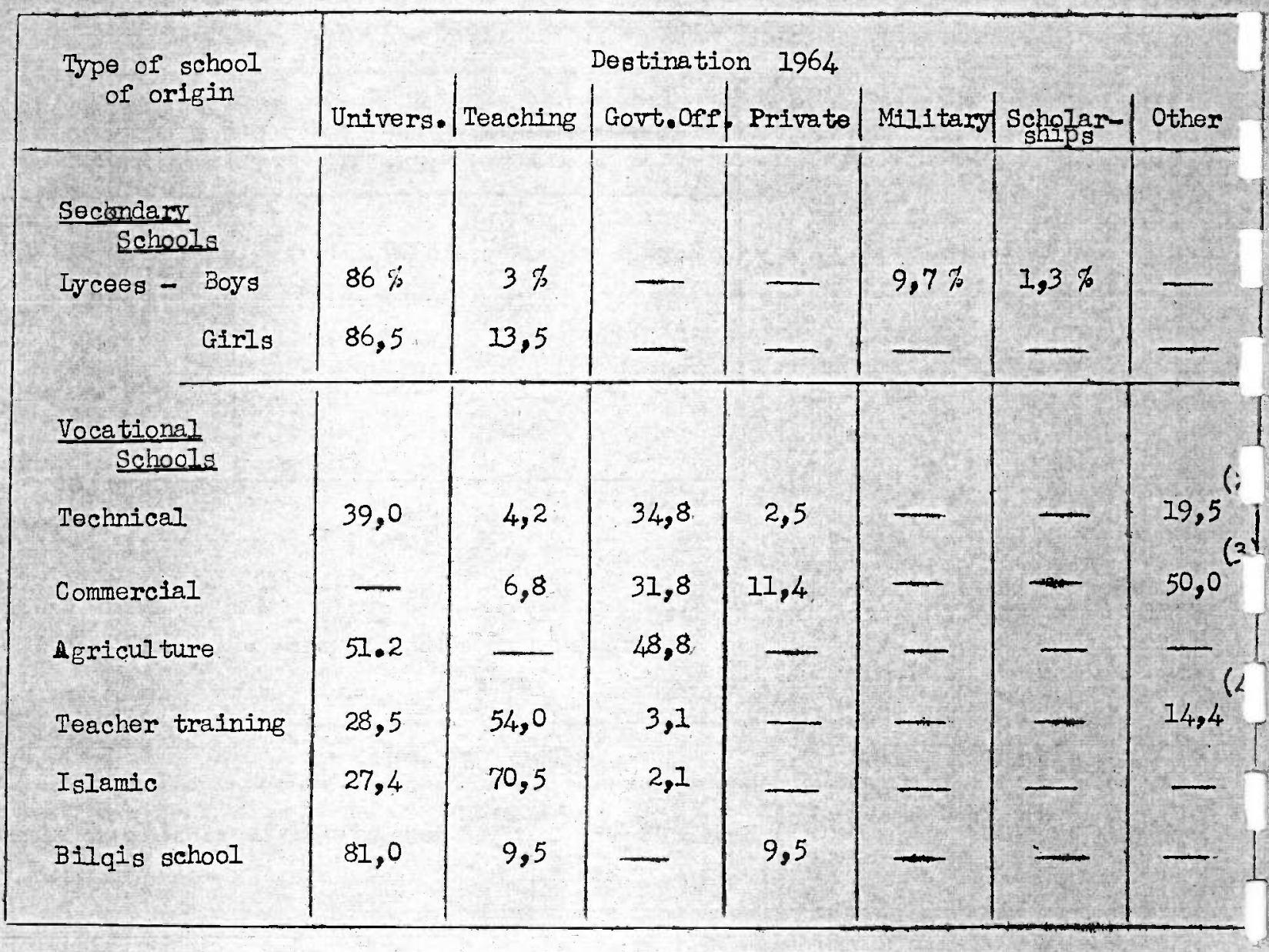

(1) See Table 37 (Source)

(2) Afghan Institute of Teshnology, Grade XIII

(3) Mostly Ingtitute of Industrial Manegement

(4) Higher Teacher Training College 
1) Enrolment

Table 47

Enrolment by sex in 1964

(1)

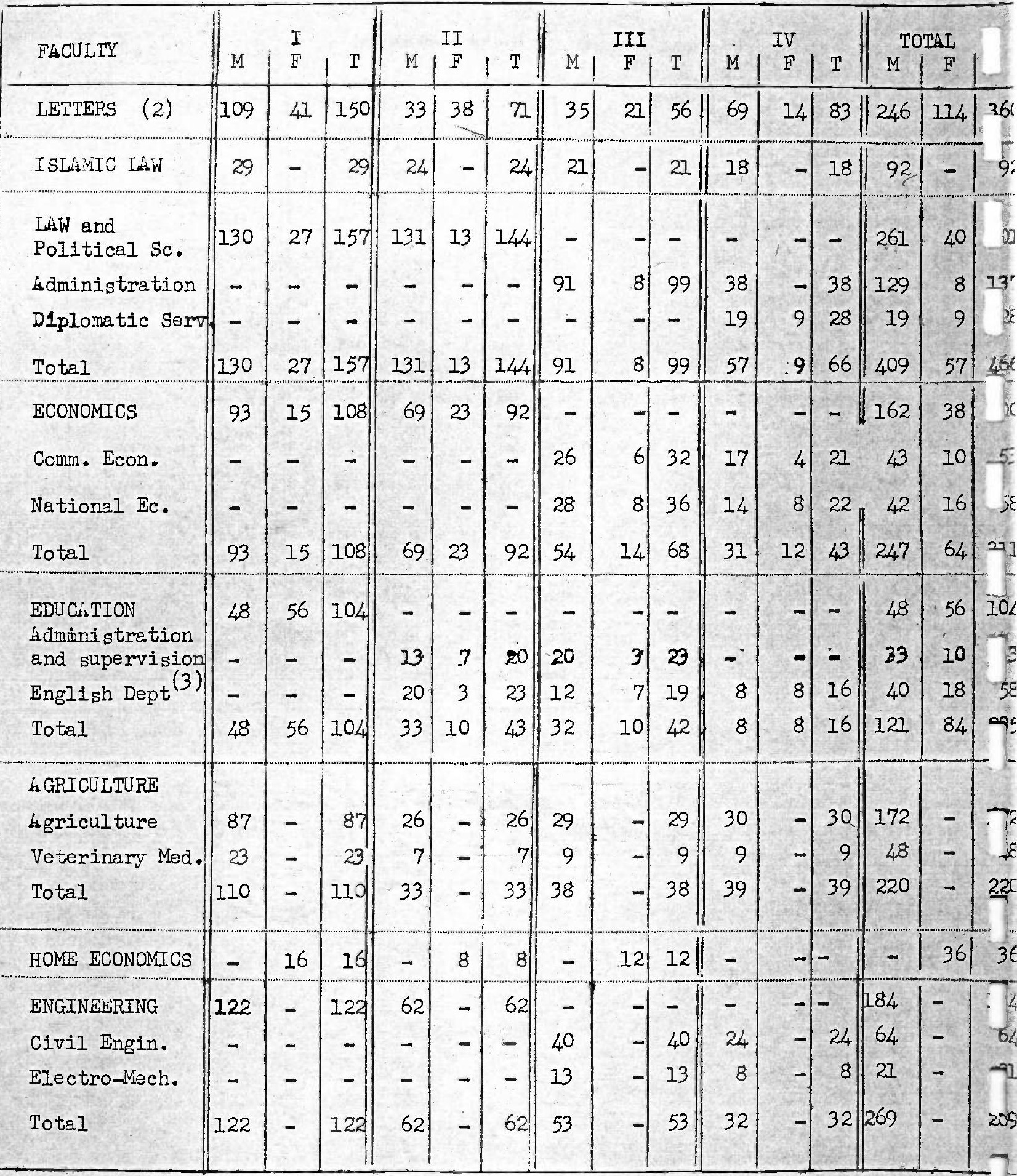

(1) See fig. 2 and page 22

(2) Figures for specialities not available

(3) Prior to 1964 the English department belonged to the Faculty of Letters 


\section{Qualifications of Teachers (1)}

Table 41.

Educational level of teachers in independent primary schools (1963) (in percentage of total).

Hither level

Grade XIII-XIV

$1 f^{\prime \prime}$

DUA

Grade XII $\quad 10$

Grade XI 5

Grade IX 19

- Other

10

$44 \%$

Grade XII

$6 \%$

$1 \%$

Vocational Schools

$0,4 \%$

Sports School

Islamic Schocls

Uncompleted Education and others
$5,6 \%$

$42 \%$

Table 42

Educational level of teachers in secondary schools (*)

$$
\text { (Kabul - City - 1964) }
$$

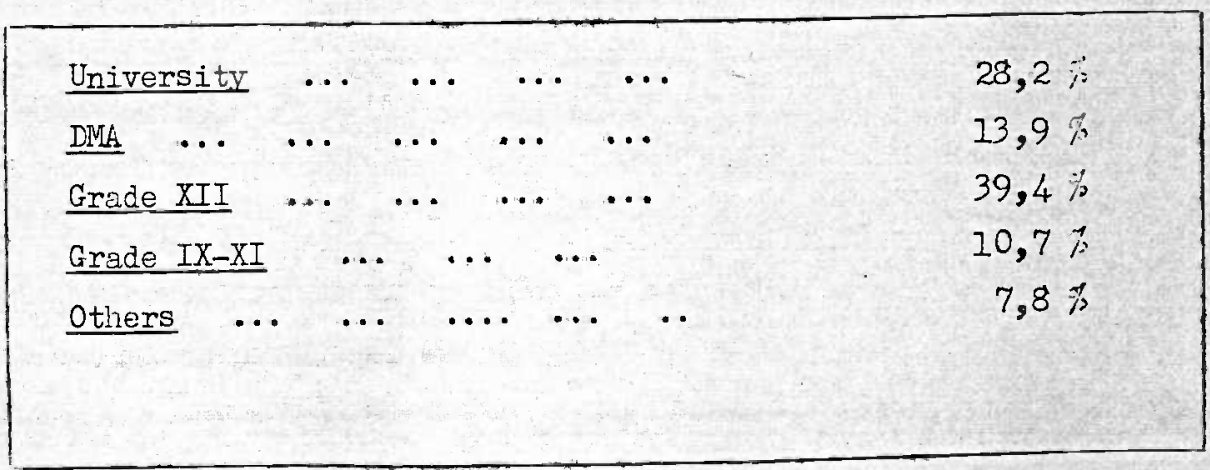

(*) Middle schools and lycees

(1) Afghan teachers only. 
Table 43

Educetional level of teachers in vocational schcols (1964)(in percentage of total).

\begin{tabular}{|c|c|c|c|c|c|c|}
\hline Types of School & University & DMA & Grade XII & $\begin{array}{r}\text { Grade } \\
\pm X-X I\end{array}$ & $\begin{array}{l}\text { Grade } \\
\text { VI_VII }\end{array}$ & Others \\
\hline Technical & 8,4 & 1,7 & 48,0 & 37,0 & 3,2 & 1,7 \\
\hline $\begin{array}{l}\text { Commercial } \\
\text {-secondary level } \\
\text {-post secondary }\end{array}$ & $\begin{array}{l}28,2 \\
100\end{array}$ & $\begin{array}{l}5,2 \\
-\end{array}$ & $\begin{array}{l}48,7 \\
-\end{array}$ & $\begin{array}{l}15,3 \\
-\end{array}$ & - & $\begin{array}{l}2,6 \\
-\end{array}$ \\
\hline Agricultural & 53,7 & 4,9 & 29,2 & 12,2 & - & \\
\hline Islomic (1) & 30,4 & 15,5 & 46,3 & 7,8 & - & \\
\hline $\begin{array}{c}\text { Technical Teacher } \\
\text { Training }\end{array}$ & - & - & 66,6 & 33.4 & - & \\
\hline $\begin{array}{l}\text { General Teacher } \\
\text { Trainine (2) }\end{array}$ & 75 & 6 & 19 & - & - & - \\
\hline Bilqis Schnol & 30,5 & 4,3 & 39,0 & 13,1 & - & 13,1 \\
\hline Ebn-Sina & 10,4 & 56,3 & 27,1 & 6,2 & - & $-\quad$ \\
\hline
\end{tabular}

(1) Bbu Hanifa only

- (2) Kabul DMA only

$3-$ Length =f Service

\section{Table 44}

Length of Service of Afghan teachers (1964)

\begin{tabular}{|l|c|c|c|c|c|c|}
\hline Types of school (1) & $\begin{array}{l}0-4 \\
\text { years }\end{array}$ & $5-9$ & $10-14$ & $15-19$ & $20-24$ & $\begin{array}{c}\text { 25 years } \\
\text { and more }\end{array}$ \\
\hline Secondary (2) & 44,9 & 19,3 & 14,5 & 9,0 & 3,9 & 8,4 \\
Technical & 60,6 & 22,4 & 4,3 & 3,3 & 5,6 & 3,2 \\
$\begin{array}{l}\text { Commercial } \\
\text {-secondary level } \\
\text {-post-secondary }\end{array}$ & 48,7 & 15,4 & 7,7 & 12,6 & 5,5 & 10,1 \\
Acricultural & 100 & - & - & - & - & - \\
Islanic (3) & 61,0 & 14,6 & 17,1 & 4,9 & - & 2,4 \\
Technical Teacher & 23,0 & 23,0 & 30,8 & 15,4 & 7,8 & - \\
General Training \\
Teacher Trainin & 50 & 33,4 & - & - & - & 16,6 \\
Bilqis School & 62,5 & 18,9 & 6,2 & 6,2 & - & 6,2 \\
Ebn_Sina & 52,2 & 39,0 & 4,4 & 4,4 & - & - \\
& 45,6 & 33,6 & 8,3 & 2,1 & 2,1 & 8,3 \\
\hline
\end{tabular}

(1) Primery schools: Data not available 
4. Pupil - teacher ratios in 1954

Table 4

Village schools..................64,3

Primary schools and primary classes

attached to secondary and vocational schools....................... 48,5

Middle schools (VII - IX)..............32

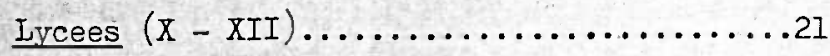

Vocational Schcols:

Teacher Training Schocls

GeneraI (X-XII).

Technical

General subjects........62

Technical subjects.......15,5

Tochnital schiols

General sukjects.........55,3

Techrical suijects........12,5

Commercial Schools

General subjects........33,3

Technical subjects........38,2

Agricultural schools

General subjects........48,9

Technical subjects.......28,2

It is important to note that the pupil-ieacher ratio and the average number of pupils per class are noticeably different in the primary scheuls. A significant number of teachers are wcrking two shifts or additional hours. The pupil-teacher ratio is 48,5 and the average size of classes is 41,1 . Table 46 gives the distrioution by provinces of the average size' of classes. 
Table 46.

Average number of pupils per class, at primary level (1964)

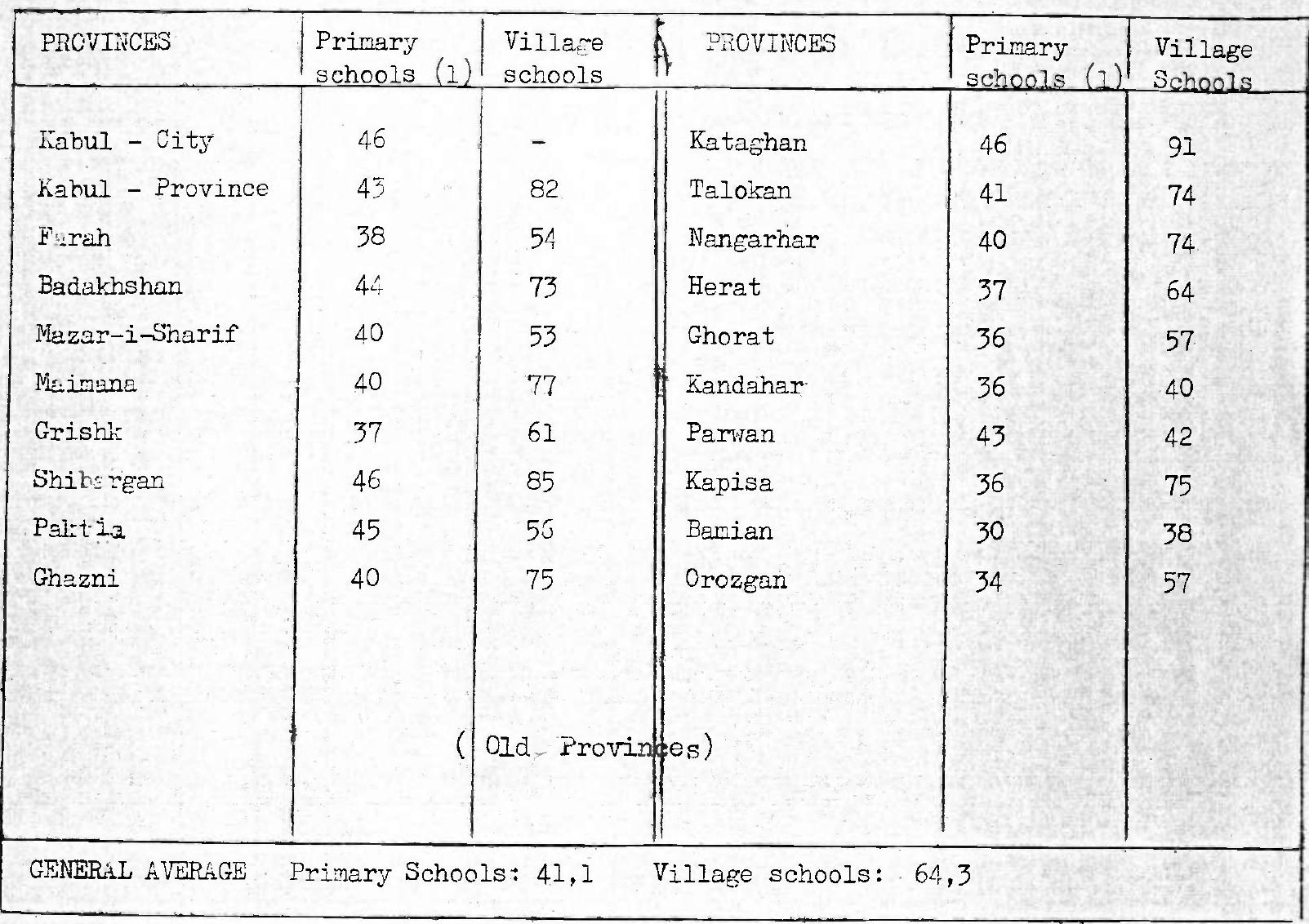

(1) Including primary classes attached to secondary and vacational schools. 
Table 47 bis (I)

\begin{tabular}{|c|c|c|c|c|c|c|c|c|c|c|c|c|c|c|c|}
\hline \multirow{2}{*}{ FACULTY } & \multicolumn{3}{|c|}{$M P C B$} & \multicolumn{3}{|c|}{$I$} & \multicolumn{3}{|c|}{$I I$} & \multicolumn{3}{|c|}{ III } & \multicolumn{3}{|c|}{ TOTAI } \\
\hline & $M$ & $F$ & $\mathrm{~T}$ & M & $F$ & $\mathrm{~T}$ & $M_{1}$ & $F$ & $\mathrm{~T}$ & M & $F$ & $\mathrm{~T}$ & M & $F$ & $T$ \\
\hline Science & 260 & 48 & 308 & - & - & - & - & - & - & - & - & - & 260 & 48 & \\
\hline Math. - Physics & - & - & - & 11 & 2 & 13 & 14 & 3 & 17 & 13 & 2 & 15 & 38 & 7 & 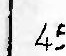 \\
\hline Chemist.-Biol. & - & - & - & 1 & 14 & 15 & 8 & 1 & 9 & 8 & 6 & 14 & 17 & 21 & \\
\hline Mineral.-Geology & - & - & - & 23 & - & 23 & 25 & - & 25 & 13 & - & 13 & 61 & - & 61 \\
\hline Meteorology & - & - & - & - & - & - & - & - & - & 4 & - & 4 & 4 & - & \\
\hline Total & 260 & 48 & 308 & 35 & 16 & 51 & 47 & 4 & 31 & 38 & 8 & 46 & 380 & 76 & 456 \\
\hline
\end{tabular}

Table 47 ter (I)

\begin{tabular}{|c|c|c|c|c|c|c|c|c|c|c|c|c|c|c|c|c|c|c|c|c|}
\hline \multirow[b]{2}{*}{$F A G$} & & $I$ & & \multicolumn{3}{|c|}{ II } & \multicolumn{4}{|c|}{ III } & \multicolumn{4}{|c|}{$I V$} & \multicolumn{3}{|c|}{$V \quad(3)$} & \multicolumn{3}{|c|}{ TOTAL } \\
\hline & $\mathrm{M}$ & $F$ & $\mathrm{~T}$ & $M$ & $F$ & $T$ & $\mathrm{M}$ & $F$ & 1 & $\mathrm{~T}$ & $M$ & $F$ & 1 & I & $M$ & $F$ & $\mathrm{~T}$ & M & $F$ & $T$ \\
\hline $\begin{array}{l}\text { PHAR- } \\
\text { MHCI }\end{array}$ & 12 & 5 & 17 & 13 & 4 & 17 & 14 & 5 & 1 & 19 & & & & & & & & 39 & 14 & 53 \\
\hline $\begin{array}{l}M E D I(2) \\
C I N E\end{array}$ & $\|_{118}$ & 22 & 140 & 83 & 22 & 105 & 57 & 9 & & 66 & 59 & 9 & & 68 & 80 & 18 & 98 & 397 & 80 & 477 \\
\hline
\end{tabular}

(1) See fig. 2, page 21 and note 7 , page 22

(2) + Faculty of Medicine in Jalalabad - Sept. 1964: 40 stadents in the first year and 40 in the second year

(3) See note 8 , page 22 
Table 48 - Global onrolment - 1962-64

\begin{tabular}{|c|c|c|c|c|c|c|c|c|c|c|c|c|c|c|c|c|}
\hline FHLCULTY & & $M$ & $\begin{array}{l}\text { I } \\
F\end{array}$ & $\mathrm{~T}$ & $\mathrm{M}$ & $\begin{array}{l}\text { II } \\
\vec{F}\end{array}$ & I & $M$ & $\begin{array}{c}\text { III } \\
\text { F }\end{array}$ & $T$ & M & $\begin{array}{l}\text { IV } \\
F\end{array}$ & $I$ & & & 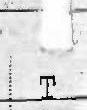 \\
\hline ters & $\begin{array}{l}62 \\
63 \\
64\end{array}$ & $\begin{array}{r}96 \\
96 \\
109\end{array}$ & $\begin{array}{l}42 \\
62 \\
41\end{array}$ & $\begin{array}{l}138 \\
158 \\
150\end{array}$ & $\begin{array}{l}69 \\
58 \\
33\end{array}$ & $\begin{array}{l}37 \\
23 \\
38\end{array}$ & $\begin{array}{r}106 \\
81 \\
71\end{array}$ & $\begin{array}{l}54 \\
68 \\
35\end{array}$ & $\begin{array}{l}26 \\
31 \\
21\end{array}$ & $\begin{array}{l}80 \\
99 \\
56\end{array}$ & $\begin{array}{l}27 \\
52 \\
69\end{array}$ & $\begin{array}{l}19 \\
24 \\
14\end{array}$ & $\begin{array}{l}46 \\
76 \\
83\end{array}$ & $\begin{array}{l}246 \\
274 \\
246\end{array}$ & $\begin{array}{l}124 \\
140 \\
114\end{array}$ & $\begin{array}{l}3 \\
450 \\
360\end{array}$ \\
\hline $\begin{array}{l}\text { Islamic } \\
\text { Law }\end{array}$ & $\begin{array}{l}6 \\
6 \\
6\end{array}$ & $\begin{array}{l}21 \\
31 \\
29\end{array}$ & $\begin{array}{l}\bar{z} \\
\overline{-}\end{array}$ & $\begin{array}{l}21 \\
31 \\
29\end{array}$ & $\begin{array}{l}21 \\
21 \\
24\end{array}$ & $\overline{-}$ & $\begin{array}{l}21 \\
21 \\
24\end{array}$ & $\begin{array}{l}15 \\
19 \\
21\end{array}$ & $\bar{z}$ & $\begin{array}{l}15 \\
19 \\
21\end{array}$ & $\begin{array}{l}24 \\
14 \\
18\end{array}$ & $\overline{-}$ & $\begin{array}{l}24 \\
14 \\
18\end{array}$ & $\begin{array}{l}81 \\
85 \\
92\end{array}$ & $\overline{-}$ & \\
\hline & $\begin{array}{l}62 \\
63 \\
64\end{array}$ & $\begin{array}{l}107 \\
150 \\
130\end{array}$ & $\begin{array}{l}10 \\
15 \\
27\end{array}$ & $\begin{array}{l}117 \\
165 \\
157\end{array}$ & $\begin{array}{r}60 \\
97 \\
131\end{array}$ & $\begin{array}{r}9 \\
9 \\
13\end{array}$ & $\begin{array}{r}69 \\
106 \\
144\end{array}$ & $\begin{array}{l}61 \\
56 \\
91\end{array}$ & $\begin{array}{r}- \\
9 \\
8\end{array}$ & $\begin{array}{l}61 \\
65 \\
99\end{array}$ & $\begin{array}{l}63 \\
61 \\
57\end{array}$ & $\overline{9}$ & $\begin{array}{l}63 \\
61 \\
66\end{array}$ & $\begin{array}{l}291 \\
364 \\
409\end{array}$ & $\begin{array}{l}19 \\
33 \\
57\end{array}$ & 39 \\
\hline Econc & $\begin{array}{l}62 \\
63 \\
64\end{array}$ & $\begin{array}{l}60 \\
96 \\
93\end{array}$ & $\begin{array}{l}16 \\
37 \\
15\end{array}$ & $\begin{array}{r}76 \\
133 \\
108\end{array}$ & $\begin{array}{l}26 \\
54 \\
69\end{array}$ & $\begin{array}{l}12 \\
14 \\
23\end{array}$ & $\begin{array}{l}38 \\
68 \\
92\end{array}$ & $\begin{array}{l}26 \\
31 \\
54\end{array}$ & $\begin{array}{l}- \\
12 \\
14\end{array}$ & $\begin{array}{l}26 \\
43 \\
63\end{array}$ & $\begin{array}{l}29 \\
26 \\
31\end{array}$ & $\overline{12}$ & $\begin{array}{l}29 \\
26 \\
43\end{array}$ & $\begin{array}{l}199 \\
207 \\
247\end{array}$ & $\begin{array}{l}28 \\
63 \\
64\end{array}$ & $\begin{array}{l}160 \\
2^{r} \\
31\end{array}$ \\
\hline Education & $\begin{array}{l}62 \\
63 \\
64\end{array}$ & $\begin{array}{l}31 \\
20 \\
48\end{array}$ & $\begin{array}{r}4 \\
8 \\
56\end{array}$ & $\begin{array}{r}35 \\
28 \\
104\end{array}$ & $\frac{\overline{6}}{33}$ & $\begin{array}{r}\overrightarrow{6} \\
10\end{array}$ & $\begin{array}{l}- \\
22 \\
43\end{array}$ & $\frac{-}{32}$ & $\begin{array}{l}- \\
10\end{array}$ & $\overline{4}$ & $\begin{array}{l}- \\
-8\end{array}$ & $\overline{8}$ & $\overline{16}$ & $\begin{array}{r}31 \\
36 \\
121\end{array}$ & $\begin{array}{r}4 \\
14 \\
84\end{array}$ & $\begin{array}{c}3 \\
5 \\
205\end{array}$ \\
\hline ¿Ericul ture & $\begin{array}{l}62 \\
63 \\
64\end{array}$ & $\begin{array}{r}56 \\
54 \\
110\end{array}$ & $\overline{-}$ & $\begin{array}{r}56 \\
54 \\
210\end{array}$ & $\begin{array}{l}31 \\
48 \\
33\end{array}$ & - & $\begin{array}{l}31 \\
48 \\
33\end{array}$ & $\begin{array}{l}28 \\
24 \\
38\end{array}$ & $\overline{-}$ & $\begin{array}{l}28 \\
24 \\
38\end{array}$ & $\begin{array}{l}29 \\
21 \\
39\end{array}$ & $\overline{-}$ & $\begin{array}{l}29 \\
21 \\
39\end{array}$ & $\begin{array}{l}144 \\
147 \\
220\end{array}$ & $\overline{-}$ & $\begin{array}{l}14 \\
147 \\
220\end{array}$ \\
\hline $\begin{array}{l}\text { Home } \\
\text { Economics }\end{array}$ & $\begin{array}{l}62 \\
63 \\
64\end{array}$ & $\overline{-}$ & $\begin{array}{l}18 \\
18 \\
16\end{array}$ & $\begin{array}{l}18 \\
18 \\
16\end{array}$ & $\bar{z}$ & $\begin{array}{r}10 \\
8\end{array}$ & $\begin{array}{r}-\overline{0} \\
8\end{array}$ & $\overline{-}$ & $\overrightarrow{12}$ & $\overline{12}$ & $\bar{z}$ & $\overline{-}$ & $\bar{z}$ & $\begin{array}{l}- \\
-\end{array}$ & $\begin{array}{l}18 \\
28 \\
36\end{array}$ & $\begin{array}{l}18 \\
2 \\
3\end{array}$ \\
\hline Engineering & $\begin{array}{l}62 \\
63 \\
64\end{array}$ & $\begin{array}{r}82 \\
102 \\
122\end{array}$ & $\overline{-}$ & $\begin{array}{r}82 \\
102 \\
122\end{array}$ & $\begin{array}{l}50 \\
47 \\
62\end{array}$ & $\bar{z}$ & $\begin{array}{l}50 \\
47 \\
62\end{array}$ & $\begin{array}{l}26 \\
38 \\
53\end{array}$ & $\overline{-}$ & $\begin{array}{l}26 \\
38 \\
53\end{array}$ & $\begin{array}{r}9 \\
18 \\
32\end{array}$ & $\overline{-}$ & $\begin{array}{r}9 \\
18 \\
32\end{array}$ & $\begin{array}{l}167 \\
205 \\
269\end{array}$ & $\overline{-}$ & $\begin{array}{l}16 \\
20 \\
269\end{array}$ \\
\hline
\end{tabular}

\begin{tabular}{|c|c|c|c|c|c|c|c|c|c|c|c|c|c|c|c|c|}
\hline \multirow[b]{2}{*}{ Science } & \multirow[b]{2}{*}{$\begin{array}{l}62 \\
63 \\
64\end{array}$} & \multicolumn{3}{|c|}{$\begin{array}{ccc}M P C B & \\
M F & T\end{array}$} & \multicolumn{3}{|c|}{ I } & \multicolumn{3}{|c|}{ II } & \multicolumn{3}{|c|}{ III } & \multicolumn{2}{|c|}{ TOTHL } & \\
\hline & & $\begin{array}{l}202 \\
240 \\
260\end{array}$ & $\begin{array}{l}49 \\
68 \\
48\end{array}$ & $\begin{array}{l}251 \\
308 \\
308\end{array}$ & $\begin{array}{l}64 \\
59 \\
35\end{array}$ & $\begin{array}{r}11 \\
4 \\
16\end{array}$ & $\begin{array}{l}75 \\
63 \\
51\end{array}$ & $\begin{array}{l}18 \\
40 \\
47\end{array}$ & $\begin{array}{l}4 \\
9 \\
4\end{array}$ & $\begin{array}{l}22 \\
49 \\
51\end{array}$ & $\begin{array}{l}31 \\
25 \\
38\end{array}$ & $\begin{array}{l}3 \\
3 \\
8\end{array}$ & $\begin{array}{l}34 \\
28 \\
46\end{array}$ & $\begin{array}{l}315 \\
364 \\
380\end{array}$ & & \\
\hline Pharmacy (2) & $\begin{array}{l}62 \\
63 \\
64\end{array}$ & & & & $\begin{array}{l}15 \\
16 \\
12\end{array}$ & $\begin{array}{l}5 \\
4 \\
5\end{array}$ & $\begin{array}{l}20 \\
20 \\
17\end{array}$ & $\begin{array}{r}9 \\
14 \\
13\end{array}$ & $\begin{array}{l}7 \\
5 \\
4\end{array}$ & $\begin{array}{l}10 \\
19 \\
17\end{array}$ & $\begin{array}{l}11 \\
9 \\
14\end{array}$ & $\frac{-}{5}$ & $\begin{array}{l}11 \\
10 \\
19\end{array}$ & $\begin{array}{l}35 \\
39 \\
39\end{array}$ & $\begin{array}{r}6 \\
10 \\
14\end{array}$ & \\
\hline
\end{tabular}

\begin{tabular}{|c|c|c|c|c|c|c|c|c|c|c|c|c|c|c|c|c|c|c|c|}
\hline \multicolumn{2}{|c|}{$(2)(3)$} & \multicolumn{3}{|c|}{ I } & \multicolumn{3}{|c|}{ II } & \multicolumn{3}{|c|}{ III } & \multicolumn{3}{|c|}{ IV } & \multicolumn{3}{|c|}{$\mathrm{V}$} & \multicolumn{3}{|c|}{ TOTHL } \\
\hline $\begin{array}{l}\mathrm{ME} \\
\mathrm{C} i \\
\mathrm{NE}\end{array}$ & $\begin{array}{l}62 \\
63 \\
64\end{array}$ & $\begin{array}{r}34 \\
89 \\
118\end{array}$ & $\begin{array}{l}11 \\
20 \\
22\end{array}$ & $\begin{array}{r}95 \\
109 \\
140\end{array}$ & $\begin{array}{l}46 \\
77 \\
83\end{array}$ & $\begin{array}{r}8 \\
10 \\
22\end{array}$ & $\begin{array}{r}54 \\
\mathrm{dx} \\
105\end{array}$ & $\begin{array}{l}85 \\
47 \\
57\end{array}$ & $\begin{array}{r}20 \\
9 \\
9\end{array}$ & $\begin{array}{r}105 \\
56 \\
65\end{array}$ & $\begin{array}{l}81 \\
88 \\
59\end{array}$ & $\begin{array}{c}13 \\
20 \\
9\end{array}$ & $\begin{array}{r}94 \\
108 \\
68\end{array}$ & $\begin{array}{l}58 \\
79 \\
80\end{array}$ & $\begin{array}{l}10 \\
14 \\
18\end{array}$ & $\begin{array}{l}68 \\
93 \\
98\end{array}$ & $\begin{array}{l}354 \\
374 \\
397\end{array}$ & $\begin{array}{l}62 \\
73 \\
80\end{array}$ & $\begin{array}{l}410 \\
447 \\
47\end{array}$ \\
\hline
\end{tabular}


Table 42.

Origin of first year students - 1963-64.

(in percentage of total)

\begin{tabular}{|c|c|c|c|c|c|c|c|c|c|c|}
\hline \multirow[t]{2}{*}{ FACULTY } & \multicolumn{6}{|c|}{$\begin{array}{l}\mathrm{KLBUL} \quad(8) \\
\text { / Voc. sch. }\end{array}$} & \multicolumn{4}{|c|}{$\begin{array}{l}\text { PUOUINCES (8) } \\
\text { | Vocat. sch. }\end{array}$} \\
\hline & (1) & $(2)$ & (3) & $\mathrm{DM}_{\mathrm{i}}$ & Others & Total & Lycee & $\mathrm{DMA}$ & others & Total \\
\hline Letters $\begin{array}{l}63 \\
64\end{array}$ & $\begin{array}{l}10 \\
4,0\end{array}$ & $\begin{array}{l}2,7 \\
2,0\end{array}$ & $\begin{array}{l}53,4 \\
54,0\end{array}$ & $\begin{array}{l}14,0 \\
18,0\end{array}$ & $\begin{array}{l}0,7 \\
4,0\end{array}$ & $\begin{array}{l}80,8 \\
82,0\end{array}$ & $\begin{array}{l}19,2 \\
18,0\end{array}$ & $\overline{-}$ & $\overline{-}$ & $\begin{array}{l}19,2 \\
18,0\end{array}$ \\
\hline $\begin{array}{l}\text { Islamic } 63 \\
\text { Law (4) } 64\end{array}$ & - & $\vec{x}$ & - & - & - & - & - & $\dot{-}$ & $\begin{array}{l}100 \\
100\end{array}$ & $\begin{array}{l}100 \\
100\end{array}$ \\
\hline $\begin{array}{l}\text { Law and } 63 \\
\text { Pol. Sc. } 64\end{array}$ & $\begin{array}{l}8,6 \\
9,1\end{array}$ & $\begin{array}{l}1.3 \\
3.9\end{array}$ & $\begin{array}{l}61,6 \\
57,8\end{array}$ & $\begin{array}{l}1,3 \\
3,9\end{array}$ & $\begin{array}{l}1,3 \\
1,9\end{array}$ & $\begin{array}{l}74,1 \\
76,6\end{array}$ & $\begin{array}{l}24,6 \\
23,4\end{array}$ & 1,3 & - & $\begin{array}{l}25,9 \\
23,4\end{array}$ \\
\hline Economic $\begin{array}{r}63 \\
64\end{array}$ & $\begin{array}{l}8,3 \\
6,3\end{array}$ & $\begin{array}{l}6,6 \\
8,8\end{array}$ & $\begin{array}{l}51,3 \\
47,0\end{array}$ & $\begin{array}{l}0,8 \\
2,5\end{array}$ & $\begin{array}{r}14,8 \\
5,0\end{array}$ & $\begin{array}{l}81,8 \\
69,6\end{array}$ & $\begin{array}{l}18,2 \\
30,4\end{array}$ & - & - & $\begin{array}{l}18,2 \\
30,4\end{array}$ \\
\hline $\begin{array}{ll}\text { Educ- } & 63 \\
\text { cation } & 64\end{array}$ & $\begin{array}{r}13,8 \\
8,5\end{array}$ & - & $\begin{array}{l}24,2 \\
58,5\end{array}$ & $\begin{array}{l}41,4 \\
11,7\end{array}$ & $\begin{array}{l}3,4 \\
6,4\end{array}$ & $\begin{array}{l}82,8 \\
85,1\end{array}$ & $\begin{array}{l}10,3 \\
14,9\end{array}$ & 6,9 & - & $\begin{array}{l}17,2 \\
14,9\end{array}$ \\
\hline Lgric. $\begin{array}{l}63 \\
64\end{array}$ & $=$ & - & ${ }^{2}, 4$ & $\begin{array}{l}2 \\
-\end{array}$ & $\begin{array}{l}83,6 \\
60,9\end{array}$ & $\begin{array}{l}87,6 \\
62,3\end{array}$ & $\begin{array}{r}12,4 \\
4,2\end{array}$ & - & $\overline{3} 3,5$ & $\begin{array}{l}12,4 \\
37,7\end{array}$ \\
\hline $\begin{array}{ll}\text { Home. } & 63 \\
\text { Econ. } & 64\end{array}$ & $\begin{array}{l}20 \\
-\end{array}$ & $\overline{-}$ & $\begin{array}{l}30 \\
25\end{array}$ & - & $\begin{array}{l}50 \\
75\end{array}$ & $\begin{array}{l}100 \\
100\end{array}$ & - & - & $\dot{-}$ & - \\
\hline Engin. $\quad 63$ & $\begin{array}{l}2 \\
1,7\end{array}$ & $\overline{1,7}$ & $\begin{array}{l}3 I, 4 \\
37,0\end{array}$ & $\frac{I}{2,5}$ & $\begin{array}{l}54,5,5) \\
48,8\end{array}$ & $\begin{array}{l}88,9 \\
90,9\end{array}$ & $\begin{array}{r}11,1 \\
9,1\end{array}$ & - & - & $\begin{array}{r}11,1 \\
9,1\end{array}$ \\
\hline $\begin{array}{cc}(6) & 63 \\
\text { Science } & 64\end{array}$ & $\begin{array}{l}16,8 \\
10,6\end{array}$ & $\begin{array}{l}3,6 \\
2,7\end{array}$ & $\begin{array}{l}44 \\
46,2\end{array}$ & $\begin{array}{r}10,4 \\
7,6\end{array}$ & $\begin{array}{r}6,8 \\
11,3\end{array}$ & $\begin{array}{l}81,6 \\
78,4\end{array}$ & $\begin{array}{l}18,6 \\
21,6\end{array}$ & - & - & $\begin{array}{l}18,4 \\
21,6\end{array}$ \\
\hline $\begin{array}{c}\text { (7) } 63 \\
\text { Pham. } 64\end{array}$ & $\begin{array}{l}15 \\
5,9\end{array}$ & $\overline{-}$ & $\begin{array}{l}50 \\
41,2\end{array}$ & $\begin{array}{l}10 \\
5,9\end{array}$ & $\begin{array}{l}15 \\
29,4\end{array}$ & $\begin{array}{l}90 \\
82,4\end{array}$ & $\begin{array}{l}10 \\
17,6\end{array}$ & - & - & $\begin{array}{l}10 \\
17,6\end{array}$ \\
\hline $\begin{array}{ll}\text { Medic. } & 63 \\
\text { Kabul } & 64\end{array}$ & $\begin{array}{l}9,3 \\
7,5\end{array}$ & $\begin{array}{l}3,7 \\
4,9\end{array}$ & $\begin{array}{l}44,8 \\
59,9\end{array}$ & $\begin{array}{l}10,3 \\
11,8\end{array}$ & - & $\begin{array}{l}68,1 \\
84,1\end{array}$ & $\begin{array}{l}31,9 \\
15,9\end{array}$ & - & - & $\begin{array}{l}31,9 \\
15,9\end{array}$ \\
\hline $\begin{array}{l}\text { Niedic. } 63 \\
\text { Jalelab. } 64\end{array}$ & - & - & $\begin{array}{c}6 \\
18,9\end{array}$ & - & - & $\begin{array}{c}6 \\
18,9\end{array}$ & $\begin{array}{l}94 \\
81,1\end{array}$ & $\overline{-}$ & - & $\begin{array}{l}94 \\
81,1\end{array}$ \\
\hline
\end{tabular}

(1) Isteqlal and Malalay lycees (Foreign language: French)

(2) Nedjat lycee (German)

(3) Other lycees (English)

(4) From Libu-Hanifa Islamic School in Bagrami, Kabul Province

(5) From if ghan Institute of Technology

(6) M.P.C.B.

(7) First year after M.P.C.B.

(8) In 1963 and 1964, for grand total: 
3) Graduates

$$
\text { Table } 50
$$

Graduates in 1962 and 1963

\begin{tabular}{|c|c|c|c|c|}
\hline FACULTY & Year & Male & Female & Total \\
\hline & 1962 & 26 & 3 & 29 \\
\hline LETTERS & 1963 & 52 & 23 & 75 \\
\hline \multirow{2}{*}{ ISLLIMIC LAW } & 1962 & 24 & - & 24 \\
\hline & 1963 & 14 & - & 14 \\
\hline LaW and & 1962 & 61 & - & 61 \\
\hline POLITICLL SCIENCES & 1963 & 60 & - & 60 \\
\hline \multirow[b]{2}{*}{ ECONOMICS } & 1962 & 29 & - & 29 \\
\hline & 1963 & 25 & - & 25 \\
\hline \multirow{2}{*}{ ISGRICULTURE } & 1962 & 28 & - & $28(2)$ \\
\hline & 1963 & 26 & - & 26 \\
\hline \multirow{2}{*}{ ENGINEERING } & 1962 & 7 & - & 7 \\
\hline & 1963 & 18 & - & 18 \\
\hline \multirow{2}{*}{ SCIENCE } & 1962 & 31 & 3 & $34(1)$ \\
\hline & 1963 & 32 & 3 & 38 \\
\hline \multirow[b]{2}{*}{$\mathrm{PH}_{i_{1}} \mathrm{RM}_{\mathrm{i}} \mathrm{CY}$} & 1962 & - & - & - \\
\hline & 1963 & 11 & - & 11 \\
\hline \multirow{2}{*}{ MEDICINE } & 1962 & 52 & 10 & 62 \\
\hline & 1963 & 59 & 10 & 67 \\
\hline
\end{tabular}

(1) 7 meleologist nod included. (2) 5 from Bérut Gaculty. 
4. Staff.

Table 51

Staff and Pupil / Teacher Ratios (1964).

\begin{tabular}{|c|c|c|c|c|c|c|c|c|c|c|c|c|c|}
\hline \multirow[t]{2}{*}{ FACLIII } & \multirow{2}{*}{$\begin{array}{r}\mathrm{A} F \\
\text { Pohand } \\
\mathrm{Mi}\end{array}$} & \multirow{2}{*}{$\begin{array}{l}\text { FGHiN } \\
\begin{array}{c}\text { Pohar } \\
\text { WoI } \\
\text { M }\end{array}\end{array}$} & \multirow{2}{*}{\multicolumn{2}{|c|}{$\begin{array}{l}\text { Di.CHING ST } \\
\text { Pohandoi } \\
M \quad F\end{array}$}} & \multicolumn{2}{|c|}{$\begin{array}{l}\text { CFF (1) } \\
\text { Yrohanmol }\end{array}$} & \multirow{2}{*}{$\begin{array}{l}\text { Poreign } \\
\text { Teach. } \\
\text { Staff }\end{array}$} & \multirow{2}{*}{\multicolumn{2}{|c|}{$\begin{array}{c}\text { Total } \\
\text { Teaching } \\
\text { Staff } \\
M\end{array}$}} & \multirow{2}{*}{$\mid \mathrm{P} / \mathrm{T}$} & \multicolumn{3}{|c|}{$\begin{array}{l}\text { Afg. Issist.Staff } \\
\text { pohanyar Fohyela1 }\end{array}$} \\
\hline & & & & & $\mathrm{M}$ & $F$ & & & & & $M$ & $M$ & $F$ \\
\hline Letters & 6 & 5 & - & - & 3 & - & 1 & 15 & - & 24 & $I$ & 11 & 2 \\
\hline $\begin{array}{l}\text { Islam:c } \\
\text { Law }\end{array}$ & - & - & 1 & - & $I$ & - & 6 & 7 & - & 13 & 1 & 6 & - \\
\hline $\begin{array}{l}\text { Law and } \\
\text { rolitical } \\
\text { Sciences }\end{array}$ & 2 & 3 & 3 & - & 4 & - & 4 & 16 & - & 17 & 1 & 8 & 1 \\
\hline Economics & 1 & 2 & 3 & - & 1 & - & 5 & 12 & - & 26 & 3 & 15 & - \\
\hline Education & - & 1 & - & - & 3 & - & $18^{(3)}$ & 22 & - & 9 & 4 & - & - \\
\hline sgricult. & 1 & - & 4 & - & 4 & - & 10 & 19 & - & 12 & 8 & 7 & - \\
\hline $\begin{array}{l}\text { Home } \\
\text { Economies }\end{array}$ & - & - & - & - & 1 & $I$ & $I^{(2)}$ & $I$ & 2 & 12 & - & - & 1 \\
\hline Engineer. & - & 1 & 1 & - & 2 & - & 7 & 11 & - & 24 & 4 & 3 & - \\
\hline Science & 7 & 5 & 4 & - & 2 & - & 7 & 25 & - & 18 & 4 & 13 & - \\
\hline Tharmacy & - & 1 & - & 1 & - & - & 1 & 2 & 1 & 18 & 1 & 5 & - \\
\hline $\begin{array}{l}\text { Medicine } \\
\text { Kabul }\end{array}$ & 15 & 13 & 20 & - & 15 & - & 4 & 67 & - & 7 & 19 & 52 & - \\
\hline $\begin{array}{l}\text { Medicine } \\
\text { Jalalabad }\end{array}$ & - & 1 & 1 & - & - & - & 1 & 3 & - & 27 & - & 3 & - \\
\hline
\end{tabular}

(1) Pohand: Professor - Pohanwol: hssoc. Professor - Pohandoi! issist. Prof. Pohanmol: Chiof lissistant - Pohanyar: issistant - rohyalai: Second issist.

(2) Female.

(j) Female 2 
Preliminary note: The figures in this section were collected at the Ministry of Education. The totals are noticeably different from tho se given in the document "Survey of irogrese $(1962$ - 1964)" by the Ministry of Planning. It is however possible to consider the data acceptable in so far as the relative proportions of the different items are concermed whether in the framework of the nationel or the Ministry of Education budget. The basic date used for the estimation of costs pelpupiz are those of the Ministry of Education.

Table 52

Educational expenditures by level of education $(1959-1964)$ in milliong of

\begin{tabular}{|c|c|c|c|c|c|}
\hline $\begin{array}{l}\text { Level of } \\
\text { education }\end{array}$ & $\begin{array}{l}1959-60 \\
\text { (Sept.-Sept. }\end{array}$ & $\left|\begin{array}{cc}1960 & -61 \\
\text { (Sept.-Sept.) }\end{array}\right|$ & $\begin{array}{l}1961 \text { (Sept.) } \\
1962 \text { (March) }\end{array}$ & $\begin{array}{c}1962-63 \\
\text { (March-March) }\end{array}$ & $\begin{array}{r}1963-64 \\
(\text { March-March }\end{array}$ \\
\hline \multicolumn{6}{|l|}{ Irimary } \\
\hline Current & 39,3 & 42,0 & 21,8 & 7,8 & 81,5 \\
\hline Capital & 4,7 & 10,7 & 2,6 & 6,5 & 32,0 \\
\hline Total & 44,0 & 52,7 & 24,4 & 78,3 & 113,5 \\
\hline \multicolumn{6}{|l|}{ SECONDIRY } \\
\hline Current & 33,1 & 45,0 & 31,2 & 43,5 & 48,7 \\
\hline Capital & 15,6 & $2 ;, 5$ & 15,5 & 11,6 & 24,0 \\
\hline Total & 48,7 & $\quad 69,5$ & 46,7 & 55,1 & 72,7 \\
\hline \multicolumn{6}{|l|}{ VQCCLTIONIL } \\
\hline Current & 36,9 & 45,8 & 13,4 & 54,9 & 59,5 \\
\hline Capital & 18,8 & 23,8 & 7,0 & 14,0 & 28,2 \\
\hline Total & 55,7 & 69,6 & 20,4 & 68,9 & 87,7 \\
\hline \multicolumn{6}{|l|}{$\underline{H} \underline{I G H E R}$} \\
\hline Current & 29,8 & 31,4 & 11,5 & 37,5 & ... \\
\hline Capital & 16,4 & 52,5 & 43,9 & 42,8 & $\cdots$ \\
\hline Total. & 46,2 & 83,9 & 55,4 & 80,3 & .. \\
\hline \multicolumn{6}{|l|}{ TOTLL } \\
\hline Current & 139,1 & $1 \in 4,2$ & 77,9 & 207,7 & $\cdots$ \\
\hline Capital & 55,5 & 111,5 & 69,0 & 74,9 & $\cdots$ \\
\hline Grand Total & 194,6 & 275,7 & 146,9 & 282,6 & $\cdots$ \\
\hline
\end{tabular}


Table 52 indicates expenditures classified by Ministry departments to which the different school establishments are attached. But as it has been seen (Table 14. p.30) a large number of pupils attend the primary classes of secondary schools. he expenses of these classest are included in the figures concerning the secondary or vacationel schools. It is therofore difficult to celculate the cost per pupil by leviels.

inother difficulty is due to the fact that expenditures for village and primary schools are grouped together, whereas in reality their costs are very different.

Finally, a difficulty is caused by the fact that whereas the school year and fiscal year coincide closely for schools with long winter vacations (school year March to Narch) but not for those with long sumner acations (school year Sept. to sept.)

f.ll these factors make it difficult to oilculate unit costs directly. Estinates have been mado as follows:-

1. Estinate of comparative unit eosts of village and primery schools based on a detailed survey in the province of Kabul. The proportion is about one to 5 or 6 .

2. Estimate of cost per pupil in village schools and independent primary schools.

2. Estimate of expenditures for pupils of primary classes attached either to secondary or vocational schools, using as a base the same unit cost as in the independent primary schools.

4. Estimate of the general cost per pupil by level of education.

The increase in the number of pupils in the course of a school yoar, in schools with long summer vacations; was taken into consideration, by basing calculations on "pupil-months".

The combination of these mothods made it possible to make an over-all estimate of current unit costs for the year 1963. (Table 53

On the basis of these costs, it is possible to estimate, for each level of education and type of institution, the real cost in proportion to total current expenditures expressed in percentages, (Table 54 ) 
Table 53

Estimated current costs per pupil in 1963

(2)

\begin{tabular}{|l|c|c|}
\hline Level of elucation & iifghanis & U.S. Dollars (I \\
\hline Village schools & 90 & 1,6 \\
\hline iriniary schools (3) & $480(4)$ & 8,5 \\
\hline Midile schools (VII - IX) & 750 & 13 \\
\hline Lycées (X - XII) & 3200 & 56 \\
\hline Vocational schools (5) & 8400 & 147 \\
\hline Teacher training (X - XII) & 3600 & 63 \\
\hline University (6) & 18750 & 330 \\
\hline
\end{tabular}

(1) Lverage rate in 1964: about 57 ifs $=1$ U.S. Dollar (free market rate)

(2) Govermment expenditures - For village schools: Salaries of teachers only

(3) Incl. primary classes attached to secondary and vocational schools

(4) Salaries of teachers: 80 to 85

(5) Excl. Teacher Training

(6) 1962 figures

Table 54

Estimated distribution of current educational. expenditures in 1963

Village schools

Primary schools and primary classes attached to secondary and vocations s schools

Middle schools (VII - IX)

Lycées

Vocational schools (excl. T.-T.)

Teacher training schools (VII - XII)

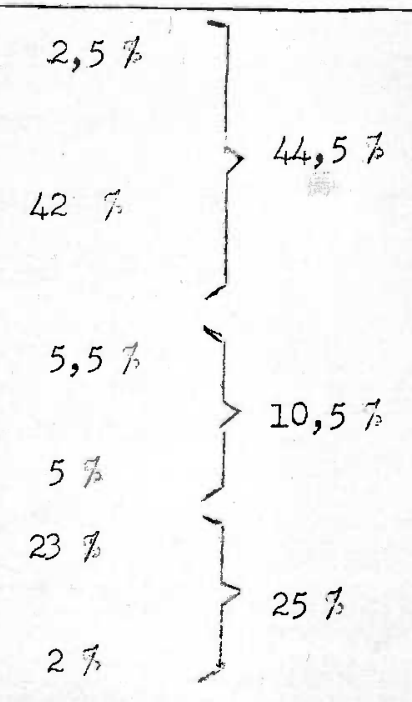

University 
Is far as unit copital casts are concorned, the following estimates have been obtcined from tho recent report of the UNiSCO Regional Idvisory Team for Pducational llanning in isia.

The se estimates can be adopted tempor:arily while awaiting the collection of more precise data on this subject.

\section{Tablo 55}

Estimated capital costs per pupil (1960)

irinary schools

Secondary schools

Vocational schools

(incl. Teacher training)

University
3000 Lff S

7500

35000

160000 
Article 34 of the new Constitution of ifghanistan states, among others, that:

.."educetion shall be provided free of charge by the state.."

ii sewhere in the same article mention is made of primary education in the Pollowing terms:

"... Is compulany for all children in the areas where facilities for this purpose are provided by the state."

Thas primary education is both free and compulsory. Young lifghan citizens, of both sexes, cain indiscriminately have access to primary education in so far as facilities for this stage are provided by the state. Genere1 Objectives:

Is stated in an official document of the Ministry of Education at the primary stage the main object is to educate and instruct every ifghan child of sohool age, providing him or her with general instruction that may prove useful in life. The final product ained at is a citizen who is a good Moslen, a staunch nationalist, and a decent sitizen of the world.

\section{Duration and conditions of enrolnent:}

The prinary stage covers a sequence of six grades. The total duration is divided into two complementary cycles; each term is composed of three consecutive grades.

The lower ago limit for enrolment lies, in principle, between the ages of 7 to 3. Primary schooling could be pursued to an upper age limit of 17 , beyond which those who "... fail to conclude their primary term of education .... aro mpelled from tho school under the provisions of the law." (3)

2. Iypes of primery education:

irimary education in $1 f_{g}$ hanistan falls into tro types:

a) Pural or village school education, and

b) Regular full primary education.

a) RuraI Educetion:

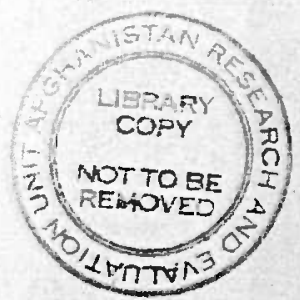

Oring to the rugged nature of the country and the consequent difficultios of communications, this type of education has been instituted in the more remote and sparsely populated areas. Thus, as long as prevailing conditions and available facilities do not permit the establishont of a full primary school a more roodest villaon ashonl is cot un inatead. 
The village school is a one-cycle school composed of the lower three grades of primary education. It is also a one teacher school that may further develop into a two-cycle, six-grade, two-teacher establishment.

Such rural education is generally given by mullahs. These mullahs are predominanty theological instructors with littie or no professional training in teaching apart from practical on-the-job experience accumiated through years of service. (1)

Where adequate school prenises are lacking it has been found expedient to locate these schools in mosques. In other places, the local people contribute funds, materials and labour to secure a more or less decent school building for their children.

It is wortly of notice in this respect that a limited number of village schools are run by the Fural Development of the Ministry of Finance under the technical supervision of the Minister of Education.

In some respects, the village school programe is modified and simplified version of the programmes of the parallel full primary school grades.

b) The full Primaly School:

The ordinary primary school is a full six-grade primary institution. It is to be found in the more populated or urban areas where available facilities permit the establishment of a regular primary school with two cycles of 3 grades each, and two or more teachers.

Buildings are either state built and owned, private houses appropriated for purposes of education through donations by individuals or local communities, or rented private houses.

Compared with the village schools, the regular prinary school curriculum is more or less advanced for the parallel grades.

It is worthy of mention that, owing to the recent unprecedented "explosion" in the enrolment, and the experienced dearth of qualified teachers to meet the requirenents of expansion, the Ministry has had to adopt a two shift system in many schools.

Further, some prinary classes are to be found attached to Middle, Lycées, and Vocational schools. 


\section{c) The Community Primary School:}

The first attempt towrds conversion of some ordinary primary schools into comunity schools began in 1960. In start was made with four schools in the provinces of Kabul and rarwan. Three more schools have since been converted, and it is boped that, by 1966 , there will be 29 pilot community schools on both the primary and secondary levels.

This conversion is effected with the help of the U.S. Igency for International Development which provides advice and some equipment and assists towards directing the operation in the right channels.

It is worthy of mention, in this respect, that the converted schools manifest a remarlable advance as compared with the ordinary non-converted schools. The conumity school is apt to play its role as a centre of cultural radiation. The activities indulged in are adapted to the needs of the environmont. Foultry, hgriculture and Handicraft projects together with sewing courses are typical activities practised in this type of school. Moreover, trips, outinss, and ficld projects help to acquaint the children with their environment. Parents and other commuity adults are inषited to these schools for various purposes.

hpart from the above each commuit gives financial support to its school whonever needed.

Convinced of whe importance of the community school and its beneficial. effect in the amelioration of social conditions in Afghanistan, the Ministry of Education has planned to convert primary schools progressively into cominunity schools all over the country. The Ministry has also planned to send study missions of Lfghan airectors of Education in the provinces, principals and teachers to the ihilippines to have first hand coniact with a similar experiment $i_{r_{1}}$ that country. On their return they will be expected to provide the converted schools with experienced and specialised staff.

Finally, reference should be made to the structure and organisation of the cormunity school project in sfghanisten. The overall project is run and controlled by a "Central Conmittee" whose chairman is the "iresident of irimary Education" in the Ministry. The Provincial Directors of Education are members of this Central Committee. 
For each irdividual school there are:

a) It "lanning Committee" which is functionally similar to the P.T.A.

b) In "lidvisory Conmittee" set up locally under the chaimanship of the District Governmor.

3. Supply of teaching materials:

There is provision in the law of the country for the supply to each chila, free of charge, of one text book covering the various subjects set for each grade. Other esseitial supaltos likewise provided by the Ministry of Iducation.

\section{Iencuares of Instruction:}

The two official languages recognised by the state as alternative media for instruction are Pashto and Dari (iersian). Wherewer more than $50 \%$ of pupils enrolled in a schocl speak either language, it is then considered the principal language and the adopted medium of instruction in all disciplines throughout the six grades. The second language $i_{s}$ initiated in the 4 th grade. It is then dealt with as a discipline among others and not as a vehicle of instruction pervading all school subjocts. This duality has its unavoidable repercussions in the preparation of text books in both languages, as well as in the preparation and distribution of teachers.

Mention should further be made of the Koran being recited in irabic during lessons of theology.

5. Examinations: (I)

To eveluate educational achiovement for purposes of promoting children from one grade to another, two complejaptary methods are used:-

- Reguatar periodic examinations of a formal nature.

- Informal day-to-day recording of pupils' activities and attainment.

(a) Periodic examinations:

These fall into three interdependent types; quarterly, halfyoarly and annual. The rosults of a pupil in the quarterly and half-yearly examinations are added up and averaged for each subject. These, averages, together with the papil's marks in the annual examinations are further averaged to obtain a final statement as to his eligibility for promotion to a higher grade. 
(b) Day-to-lay records:

These informal appraisals of the pupils' activities aro recorded In the class teacher's book, added up for each month, and later taken into consideration in determining the pupil's standing in the quarterly and helf-yearly examinations.

The quality of schools and the number of the them has been observed to be related to the available transport facilities.

\section{General Remarks:}

I. The education of girls in the Pashto speaking areas seems to be lagging behind that of other areas.

2. Information was conveyed to the Tean of the existence in most villages of a considerable number of traditional type schools located in mosques (1) These pseudo-schools do not belong to' primary education as such. The children enrolled are naturally not included in the statistics for the proper primary level though a considerable number of children are involved. by

This typo of school is established and privately mun mulahs espociallwhere no regular primary education is provided by the state.

Instruction is mainly religious and is based on the Holy Koran. I start is made with Kaidat - ul - Baghdadi beginning with the Srabic alphabct, Irabic word composition and culminating with routation of verses from the Jecred Book.

Writing and irithmetic are rarely taught unless it be through the initiative of a relativly experienced Mllah.

In this respect it is interesting to note the following:-

a) Some children may continue in that direction receiving from the vilizia further and nore advanced training in religious discipline such as LI-FIRT, MI-WHWM, LIMUNTIK, MUMMLUT and others. This ultimately leads to a mullah career for the young aspirants. It also opens the way to other state functions especially related to Islamic Law.

(1) See The Sleight Report, p. 28 where an estimate of 250,000 is tentatively given as the probable enrolment in 1962. 
b) Wherever and whenover facilities become available the State stops in to convert this modest form of schooling into a

state rur village school or even a full primary school.

\section{$T L_{1} B L \equiv 56$}

WEEREY TIIE-TEBIE FOR XESHTO-SIEHKTNG SCHOOLS

\begin{tabular}{|c|c|c|c|c|c|c|}
\hline \multirow[t]{2}{*}{ SUUBJECTS } & \multicolumn{3}{|c|}{ PILST TERM } & \multicolumn{3}{|c|}{ SECOND TERM } \\
\hline & Class I & Class II & Class III & Class IV & Class V & Class VI \\
\hline $\begin{array}{l}\text { Holy Qoran } 3 \\
\text { Theology }\end{array}$ & 4 & 4 & 4 & 4 & 4 & 4 \\
\hline Pashto & 12 & 12 & 12 & 5 & 5 & 5 \\
\hline rersian & - & - & - & 5 & 4 & 5 \\
\hline $\begin{array}{l}\text { Writhmetic \& } \\
\text { Geometry }\end{array}$ & 4 & 4 & 4 & 4 & 5 & 5 \\
\hline History & - & - & - & 2 & 2 & 2 \\
\hline Geography & - & - & - & 2 & 2 & 2 \\
\hline $\begin{array}{l}\text { Natural } \\
\text { Science }\end{array}$ & - & - & - & 2 & 2 & 2 \\
\hline $\begin{array}{l}\text { Prawing \& } \\
\text { Handicrartus }\end{array}$ & 3 & 3 & 3 & 2 & 2 & 2 \\
\hline Caligraphy & 4 & 4 & 4 & 2 & 2 & 1 \\
\hline $\begin{array}{l}\text { hysical } \\
\text { Training }\end{array}$ & 1 & 1 & 1 & 1 & 1 & $I$ \\
\hline Totel & 28 & 28 & 28 & 29 & 29 & 29 \\
\hline
\end{tabular}

c) fidult Education

To neet the problen of illiterate adults, courses are provided by the Government in many partis of the country. In 1964 there were approximately 300 classes operating. They are mainly held at the local primary or middle school. 


\section{UTCONDARY SCHOOLS}

The secondary schools of iffghanisten consigt of two cycles, the lower cycle or middle schoel (erades $7-9$ ) and the upper cycle or lycée (Erades 10- 12). Very often the term lycée is used to signify the whole of the secondary school (grades 7-12). $\therefore$ s in most cases a primary school cycle (grades 1 - 6) is atteched to the secondary school, the term. may, in fact, also mean a school with grades I - 12. snother difficulty of terminology arisis from the fact that many vocational schools belones to the secondary level and sometimes are included in the concept "secondary schools".

Here however we use the words secondary school, middle school and lycée only in the following sense:

1. Secondary school: Grades $7-12$ in schools with a curriculum for capral education without vocational specialization.

2. Middle school: Grades $7-9$ in schools with the above-mentioned reneral curricuzum.

3. Iycé: Grades $10-12$ in schools with the above-mentioned curriculum.

The curriculum in girls' schools is slightly different from that in boys' schools. 


\section{T.BLE, 57}

Weekly hours for secondary schools for boys.

Subjocts

Gretes

$\begin{array}{llllll}7 & 8 & 9 & 10 & 17 & 12\end{array}$

"hoolong \& The

Holy Koran

$\begin{array}{llllll}2 & 2 & 2 & 2 & 2\end{array}$

6

4

10

Pashto (iashto-

speaking regions) $4 \quad 4 \quad 4 \quad 4 \quad 4 \quad 3$

íashto (Dari-

$\begin{array}{lllllll}\text { speakinf regions) } & 3 & 3 & 3 & 3 & 3 & 3\end{array}$

Dari (Dari-

speaking regions) $4 \quad 4 \quad 4 \quad 4 \quad 4 \quad 3$

Dari (rashto-

speaking regions) $3 \quad 3 \quad 3 \quad 3 \quad 3 \quad 3$

isithmetic \&

Geometry

History

$\begin{array}{llllll}5 & 5 & 5 & 7 & 7 & 7\end{array}$

$\begin{array}{llllll}2 & 2 & 2 & 2 & 2\end{array}$

Drawing \&

Handicrafts

Chemistry

Pysics

1111

$\begin{array}{llllll}2 & 2 & 2 & 3 & 3 & 3\end{array}$

Geology

$\begin{array}{llllll}2 & 2 & 2 & 3 & 3 & 3\end{array}$

Geography

Biology

Lrabic

Foreign language

General knowledge-

Economics 111

Religion

Logic

sports

Total +

111121
Totel

ialis scivol yycóo
11

23

18

12

11

23

18

15

21

36

6

12

3

3

6

$=15$

6

6

12

6

6

18

34

+ The totals include iashto and Dari accordine to the curriculum oither for $i^{3}$ shto-speaking or Dari-speaking regions. 


\section{$\underline{T+B L E} 58$}

Weekly hours for secondary schools for girls.

Subjects

Grades

$$
\begin{array}{llllll}
7 & 8 & 9 & 10 & 11 & 12
\end{array}
$$

Theology

$\begin{array}{llllll}2 & 2 & 2 & 2 & 2 & 2\end{array}$

rashto (iashto-

speaking regions) 34445

iashto (Dari-

speaing regions) $3 \quad 3 \quad 3 \quad 3 \quad 3 \quad 3$

Dari (Dari-

spaking regions) 34445

Dari (rashto-

speakin regions) $3 \quad 3 \quad 3 \quad 3 \quad 3 \quad 3$

Foreign Language $\begin{array}{llllll}6 & 6 & 6 & 6 & 6 & 6\end{array}$

History

Geography

Nathematics

Geometry

hysics

Chemistry

Biology

Sewing

Drawing

irabic

Total +

$\begin{array}{llllll}2 & 2 & 2 & 2\end{array}$

$\begin{array}{llllll}2 & 2 & 2 & 2 & 2 & 2\end{array}$

$\begin{array}{llllll}2 & 2 & 2 & 2\end{array}$

$\begin{array}{llllll}3 & 3 & 3 & 3 & 3 & 3\end{array}$

$\begin{array}{llllll}2 & 2 & 2 & 3 & 2\end{array}$

$\begin{array}{llllll}2 & 2 & 2 & 3 & 3 & 3\end{array}$

$\begin{array}{llllll}2 & 2 & 2 & 3 & 2 & 3\end{array}$

$\begin{array}{llllll}2 & 2 & 2 & 1 & 2 & 2\end{array}$

$\begin{array}{lllll}2 & 1 & 1 & 1 & 1\end{array}$

222 $\begin{array}{cll}\text { Total } & \text { Total } & \text { Grand } \\ \text { Middle school } & \text { Lycée } & \text { Total }\end{array}$

$\begin{array}{lll}6 & 6 & 12\end{array}$

11

14

25

18

11

14

25

18

$\begin{array}{rrr}9 & 9 & 18 \\ 18 & 18 & 36\end{array}$

$6 \quad 6 \quad 12$

$6 \quad 6 \quad 12$

$6 \quad 6 \quad 12$

$9 \quad 9 \quad 18$

$6 \quad 7$

699

$6 \quad 8 \quad 14$

$\begin{array}{lll}6 & 5 & 11\end{array}$

42

6

+ The totals include Pashto and Dari according to the curriculum either for ishto-speaking or Dari-speaking regions. 
In addition to the periods given in the chericula six periods per week are set aside for practical and experimental work in science in the lyc6es. There is some doubt as to whether these periods are in fact always used.

The foreign lanjiage taught in practically all schools is English. In the Isteclal boys' school and the Malalay girls' school in Kabul the foreign language is, however, French, and in the Nedjat boys' school, also in Kabul, German is taught. In Rahman Baba boys' school in Kabul it is to. some extent possible to choose between English and German.

The teaching language in the secondary schools is either Dari or rashto. In the Isteclal school some subjects in the lycée are, however, taught in French.

The present syllabuses are very much like the traditional European secondary school syllabuses used for the science and mathematics streams in the $1930^{\prime} \mathrm{s}$. Normally there are no general or humanities streams, and no optional subjects. The goal of the middle school syllabus is to prepare for the lycé, and the goal of the lycée syllabus to prepare for the university. +

The secondary schools are very much concentrated in Kabul. Out of the 26,352 pupils enrolled in the secondary schools (gr. VII - XII) in 1964 13,610 (more than half) belong to the schools in the capital. Taking into account only the lycés (gr. X - XII) the distribution is still more disadvantageous to the provinces: out of 4,528 pupils 3,391 belong to the Kabul schools. It should, however, be noted that two of the Kabul secondary schools, the Rahman Baba and the Koshal Khan school, are boarding-schools with pupils mainly from provincial areas. The se schools hate an enrolment of 1,I01 pupils in 1964, of whom 302 belong to the Iycée (gr.X - XII). The running of these schools is in accordance with the recommendations of the General UNESCO Conference in 1960 that hostel facilities should be made available in urban areas for pupils from rural areas in those cases where the distribution of schools was upfavourable to children in rural areas.

Even taking these boarding facilities into account it must be stressed that the distribution of secondary schools is most unfavourable to the provinces.

The lycée finishes with an examination called the baccalauréat with written and oral tests. For the written tests four proposals are given by each teacher. Out of these proposals the Ministry of Education decides about the tests to be given to the pupils. For the oral examination the tests are given by the teachers. The

+ Raf: Ministry of Education, Syllabuses secondary schools, Kabul 1956.-Ministry of Education, Education in Lfghanistan, Kabul 1956 (printed in München, Germary). is these two printed references are both eight years old, supplementary information has been given by the officials of the M istry of Education.

++ For details about the distribution of schools see above p. 3I, table I7. For the UNESCO recommendations see Report of Meeting of Ministers of Education of isian Member States Participating in the Karachi Flan, Bangkok 1962, p. 18.

The figures for the secondary schools mentioned here do not include the enrolments in the Kabul knginine lycée. See $\mathrm{p}$. 
oral examination is supervised by inspectors from the Ministry and by special inspectors appointed by the Ministry, these latter usually being lycée teachers.

At the end of the ninth grade an examination is arranged in almost the same way as for the twelfth frade, only that the tests for the written examinations in the provinces are decided upon by the provincial directors of education.

All secondary school education is free, and there is legal provision for the supply of writing materials.

In the provinces there are five boarding-schools for Middle School boys, and in Kabul City there are, as mentioned above, two such boarding-schools, including both middle school and lycée.

+ The scale of marks goes from 1 to 10 , 10 being the highest mark.

\section{School Vacations.}

Because of the high fuel costs, the schools in those psris of the country where the clinate is rather severe have winter vacation, whereas summer vacation $i$ s prevalent in the other parts of the country.

The Winter vacation begins on Acrab 15 (Nov. 6.) in the primary schools and on Kaws 15 (Deć. 6) in the secondary and vocational schools. This vacation lasts till Houd 15 (March 6).

The Summer vacation begins on Jawza (June 5) and ends on Soumboula 15 (Sept. 6) in prinary, secondary, and vocational schools.

In $196367 \%$ of the schools had winter vacation and $33 \%$ summer vacation. 
gubjoots German, inglish, oconomical geography, technology of raw-matetials Wh prothlour, rexclipsions to indugtrial undertakings with discussion, optional practical work in differen's fields of commercial techniques, commercial arithmetic with practicum, book keeping with practice, commercial law and Dari typewriting. is practicum in industry in the vacations following the first class courge is compulsory:

The second and third years of training cover the necessary industrialcommercial subjects with rich practical work as: practice in commercial techniques, calculation with practice, process planning with practicum, management, statistics, Gemen for advanced students, Geman commercial correspondence, Inglish ccmmercial correspondence, German typewriting etc.

In the vacations followire the second year a training of 2 months duration ir industrial undertikings is performed. Neter the third and the final theorotical examination a five months practical training in industry is prescribed. Then the student; may be admitted to the practical final examination.

\section{3. isgriculture Schoolse}

is at present about $90 \%$ os the population of lifghanistan are engaged in farming (including nomads) the development of agricultural eduoation is most important.

Bilateral aid in agricultural education is provided by the USAID - team (United States igency for International Development) of the Univeristy of Wyoming.

There are four independert administrations for agricultural education in : the country:

a). The Ministry of Educaiion, Department of Vocational Education,

b). The Ministry of Agriculture,

c), The Rural Development Department,

d). The Helmand Valley Luthority.

Lgricultural schools may belong to any of these authorities. The Royal Ministry of Education is providing textbooks, approving ciricula and carrying out inspections for all these schools.

igricultural education in primary schools is given by qualified teachers in agriculture, who must be graduates of the Lgricultural College, or by semi- . qualified teachers who are Graduates of Teacher Training Colleges trained in agriculture (or teachers of the onergency teacher programme or teachers who have finished a three months course of Teacher Training in Lgriculture). The 


\section{Multiform Schools}

Irincess Bilgis Girls 3chool, Kabul, grados VII - XII, trains girls for secretarial and office work but also gives theoretical and practical training in home economics. Graduates of the schoel may continue their studies in difforent Faculties of Kabul University, especially in the Economics and HomeEconomics Faculties and in the Industrial Manngement Institute.

The curriculum covers geography, history, law, economics, book-keeping, human relations, business mathematics, arithnetic, algebra, geometry, ethics, Cieligion. its shto, Dari, English, physics, social sciences, chemistry, biology, Dari typewriting and theoretical and practical home-economics subjects.

Ebn-i-Seena vocational middIe school, grades VII - IX, Kabul, is preparing pupils in the first line for entrance into different vocational schools. Its curriculum not only covers the subjects common to other middle schools but gives also practical training in handicrafts and agriculture. 
The D.W.A.'s are under the contrul of the Directorate of Teaner Education in the Iepartment of Vocational Education. The senior cycles (Grades X, XI and XII) besides providing formal teacher training for primary and middle school teaching, offer the normal lycee course for selected students proceeding to the University of Kabul for training as lycee teachers. The curriculum for the student trainees is as follows:$\cdot$

Periods per week

$$
\text { Grades }
$$

$\begin{array}{llll}X & X I & X I I & X I I \\ & - & \text { Primary Trainees } & \text { Faculty Prainees }\end{array}$

1. Peda,gogical Subjects

(iducation, Psychology) $33 \quad 3 \quad 3.26$

\section{Teaching Practice}

(including Gulzar

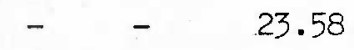
programme and community schools)

2. Languages

$\begin{array}{lllcl}\text { Afghan } & 6 & 6 & 4 & 6 \\ \text { Foreign (English) } & 4 & 6 & 1.6 & 6\end{array}$

3. Social Studies

(History, Geography 6 General Knowledge, Economics)

4. Mathematics

46

1.6

5. Sciences

6. Apriculture

7. Health Education

3. Arts and Crafts (including Handwriting)

9. Theology

$\begin{array}{lll}9 & 6 & 2.3\end{array}$

$\begin{array}{llll}4 & 2 & - & 2\end{array}$

Total

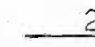

41

$-1$

33

1.66

2

$3 \quad 1.66$

1

2

41

* includes 4 periods in Logic and Philosophy.

During the past ten years the D.M.A.'S have received considerable assistance in building and equipment from U.S.A. and in professional help and guidance from the Colurnibia Team of USS/AID.

During the period 1958-62 the average number of graduates (grade XII) from the four D.M.A.'s was 78. In 1962, out of a total of 451 grade XII graduates from vocational schools $94(21 \%)$ received specific training as teachers for prinary and middle schools. (Regionel Survey - Table XIV - Page 37.)

(1) In 1964 the training of teachers for middle schools became the specific function of the Higher Teachers College - see section II (a) 
During the same period (1958-62) the average increase in the number of teachers in primary and village schools was 212 per year. (Regional Survey Tables I and II - Page 24 and 25). In 1963 the additional number, employed was 590 ( 192 in village schools and 498 in primary). (Regional Survey Tables $I$ and II - Page 24 and 25).

(Note. These figures exclude the number of teachers engaged in teaching primary classes in middle, lycee and vocational schools.)

Assumine that $75 \%$ of the teacher trainees (70) from the D.M.A.'s entered the primary service the balance (620) employed were untrained. In other words apjroximately $10 \%$ of the teachers entering the primary school service in 1963 were trained teachers and this is in accord with the general pattern of the qualification of teachers.

(b) EMLRGLICY TEACHER TRA INIIVG SCHEME.

To meet the need for teachers for the expending primary schools, an emergency training scheme was introduced in 1952 under the guidance of the Columbia University Team of US/AID. The scheme provides a three year course in general education at grades VII, VIII and IX, to be followed by a one year course of professirnal training (grade $X$ ) to be provided at the existing D.M.A.'s Students are paid a monthly allowance of $100 \mathrm{Afs}$. (grade VII) $150 \mathrm{Afs}$ (grade VIII) and $200 \mathrm{Afs}$. (grade IX) in addition to the provision of clothing and books.

Although the scheme was originally intended to operate for one year's enrolment, students have keen enrolled in 1963 and 1964. The enrolment in 1964 is as follows:-

$\begin{array}{llll}\text { Grade VII } & \text { Grade VIII } & \text { Grade IX } & \text { Total } \\ 1168 & 941 & 606 & 2715\end{array}$

Groups of emergency trainees were arranged in classes of 35 attached to middle and lycee schools throughout the provinces. (the number of students enrolled are included in Table 16 - Page 31.)

In 1962 twenty nine groups ( 16 being day and 13 boarding students) were established, representing a total enrolment of 991 . $60 \%$ of the students came from areas where rari is the main language and $40 \%$ from Pakto speaking areas. Similarly $60 \%$ are from sumer vacation areas and $40 \%$ from winter areas. It is estimated that 606 students will complete Grade IX in $1964-65$ school year, of whom 258 will commence their year of professional training in March 1965 and 348 in September 1965.

In general the curriculum followed by the emergency trainees has been substantially that of the normal middle school grades. (I)

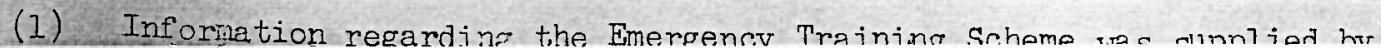


SCADITY OF THACHER TRAINING.

This is a UNESCO/UNICEF sponsored project, established in 1964 and designed to train teacher educators to staff primary teacher training colleges. At the same time the demonstration training college which forms an essential workine part of the Academy offers a three year course in general education and professional preparation for primary teachers. Associated with the Acadeny is a Primary Demonstration School. Ninety students (all male) were enrolied in 1964, and it is anticipated that the Academy will prepare up to 130 primary teachers per year when it is fully established. The Academy is designed as a co-educational irstitution. For further reference see section $\sqrt{5}$.

The lanned weekly tiretable of the student trainees is as follows:Weekly Time Table of Student Trainees at the Academy

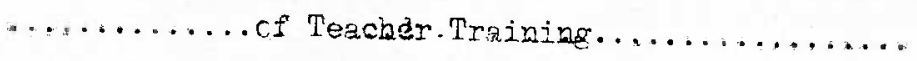

1. Pedagorical Subjects

(Education, Psychology, Principles of Teaching)

Periods per week X Grades XI XII

Teaching Practice

2. Languages.

Afghan

Foreign (English)

3. Matr ematies

4. Sciences

(a) General Science

(h) Rural Science (Men) Domestic Science (ifomen)

6

6

6 weeks 8 weeks

12 weeks

\section{Social Studies}

(History and Geography)

\section{Physical Education}

(including health education)

7. Music

8. Arts and Crafts

9. Islamic Studies

10. Study Periods

$\begin{array}{lll}5 & 5 & 5 \\ 4 & 6 & 6 \\ 4 & 4 & 4\end{array}$




\section{(d) WOMEN TEACHERS}

Frcvision for the education of girls has been expanding at a rapid rate in recent years (see pages $36,37,38$ ). The enrolment of girls in Frimary Schools rose from 10,012 in 1956 to 28,405 in 1982. During the same period the number of women teachers emplcyed in village schools rose from 3 to 96 , and in prinary schools from 162 to 492 (Regional Survey Tables 192 Fages 24 and 25). In 1963 the additional number of women teachers in primary and village schools was 144 .

Facilities for the professional training of women teachers for primary schools are provided through in-service courses. It should be noted, hnivever, that the two UNESCO sponsored training colleges - the Higher Teachers' College and the Academy of Teacher Training are both designed as co-educational institutions.

(e) IN-SERVICE TRAINING

The recruitment of teachers for primary schools has, for many years, been drawn from untrained or undertrained personnel. To compensate in some measure for this lack of training, professional guidance has been available from the תepartment of Supervision and Inspection, and from in-service courses. Winter and summer vacation courses and short courses organised mainly by the Institute cf Education, with assistance from the Columbia Team, have provided opportunities for professional training and an improvement in the general level of educational attainments. These courses commenced in 1960 when 60 women teachers enrclied in the summer course; 317 men and 505 women enrolled in the winter course. In 1964 sessions 141 women attended the summer course; 398 men and 110 women attended the winter course. Instruction is offered at two levels - D.M. L. level (Grades 9-12) and University faculty level.

\section{SECONDAPY THACHER TRATIING.}

(a) MIDDLE SCHOOLN

Training facilities for teachers at the Middle School level, as indicated above, have keen provided ry the D.M.A.'s. This was limited to graduation at Grade XII level. To raise the qualifications of teachers for middle schools and to meet the crucial need for fully trained teachers at this level, the Higher Teachers' College was established in Kabul in 1964. This is a Special Fund Project, sponsored at the request of the Government, by UNESCO. 
The project was designed to provide a two-year course beyond Grade XII with specialised teacher training in the sciences (including mathematics) and in the humaities (languages and social studies). The College is a cc-educational institution ard the enrolment of students in 1964 (28 men and 2 women) was, for various reasons, far below the anticipated enrolment. (b) LYCEES.

Teachers for lycees (erades X, XI and XII) are drawn from various sources, the Iniversity of Kabul being regarded as the main preparatory irstitution for teching at this lerel. University graduates from the Faculties of Science and Letters regard teaching in the Lycees as one of the main vocations open to them. However, the number of University trained personel in these schools is very low. Pedagrgical training for university graduates wishing to teach is at a minimum although it is not uncommon for University students to engage in part time teaching in a secondary school and gain some teaching practice.

The Facuity of Educ tion offers specialised pedagogical courses based cn the liberal arts. In 1764 the teaching of English became a department of the Froulty of Educution and is responsible for the training of English Language teachers. Asscciated with the Faculty of Education is the Institute of Eduction which as indicated above, has made many contributions in the field of teacher training.

3. TECHIICAL THACHER TRAIIIIIG.

Teachers for various branches of technical education receive their preparation and truining at the Techn_cal Teachers College in Kabul at Grades $X, X I$ and XII. Students in these courses are drawn from the top pupils at the mechanical schocls of Kabul, Kandahar and Khost and from the craft schools in Kabul and Farah. Their training includes an extension of their studies in their particular field of general mechanics, electricity or construction, together with some training in the art of teaching. The number of students in grade XII in 1963 was 29 , of whom 20 entered teaching in 1964 . The numer of students in grade XII in 1364 is 32.

This section of teccher training receives assistance and guidance under the bi-lateral aid from the Federal Remblic of Germany.

4. OTHLR FACILITILS FOR TEICFER TRIINING. (a) Sports School. This institution provides a training and preparation. for teachers of physical education in the schools. The number of pupils enrolled in this school is shown in Table 18 
(h) Islawic Studies.

-ce Section III - paragraph 5.

(c) Art Teachers.

\section{A speciel courat for the training of hrt teacherm it attached to the}

Craft School in Kabul. Students complete a three year course (grades X, XI and XII) in the field of art and crafts. See Section III - paragraph 1. (d) Home Economics.

In the curriculum of the girls' schools provision is made for some instruction in home economics. At present there are no trained teachers in this field, is the University of Kabul there is a Faculty of Home Economics frum which it is anticipated some trained teachers will be recruited. The first class will graduate in 1965.

5. THACHER EDUC TORS.

Nu description of teacher training facilities wuld bo complete without reference ts the provision for the training if personnel to staff teacher training institutions. To supplement and complement the preparation of teacher educ.tors provided by the Faculty of Educ tion, the UNESCO/UNICEF sponsored project, the Acadelly of Teacher Training. was established in 1964. This institution has a unique function in prividing a direct practical training for teacher educators. A primary training cullege with a three year course serves as a demonstratiou training college for teacher education trainees whe are university graduates with some teaching experience. The tescher educator trainees spend one year at the Acadcmy, teaching and guiding the students of the training uollege, under the superision of an internationally recruited staff. Their course, at postiniversity graduate level, includes studies in the theory and practice of education, English laiguage and special methudolugy. In the Demonstration School attached to the Acadeily, they leam $h: w$ to direct and supervise students in the prictical arts of teaching. After graduating from the Academy, the teacher educators are available to staff training colleges throughut the country. It is estimated that when the Academy is fully developed there will we an cutput of 62 techer educators per year, and 130 trained primary teachers. The Academy is a co-educational institution at both levels.

In order to mect the need for teacher educators for the emergency trainees, the Faculty of Education urganised cuurses in 1963 and 1964 for teachers of prufcssionsl sujects. In 1963 a group of 15 students completed a year of training and in 1964 a group of 20 studints participated in the course. 
6. SPECIALIST TRAINING.

At the time of preparation of this report there are no organised training facilities for the following:-

(a) Kindergarten Teachers.

The develojment of pre school educati n for children in city areas is developing.

(b) Adult education, particularly associated with the eradicaticn of illiteracy requires specialist training of teachers. Much experimental work has heen done in this field in various parts of the world. In the training of prinary teachers at the Kabul D.M.A. provision has been made for students to visit and study some of the methods employed in the community schools and in the training of village workers at the Gulzar Training Centre.

(c) Handicaoped Children.

In every community there is a need for providing a suitable education for the physically and intellectually handicapped children. There are no state provisions for specialised training in these fields. 
Higher Education is given in the Faculties of the Kabul University. The Kabul Jniversity was formally inaugurated in 1946, but its first faculty, that of Medicine, was founded in 1922.

$\therefore$ s shown by $F i g$. 3, Organisation Chart of the loyal Ministry of Education, in this brochure, the University Ldministration is not dependent on any Department of the Ministry of Education but directly responsible to the linister of Education.

The different faculties of the University are registered in the diagram Fir. 2, Organisation of the educational system. There is co-education in all faculties except in those of -ngini eering, Islamic Law, and Home Economics. By the end of 1963 all faulties had been transferred from formerly rented buildings in the City to the University Campus at ili libad, in the Western ou skirts of Kabul. In 1963 a University College of liedicine was established in Jalalabad. The Faculties confer the Bachelor's degree at the end of the fourth year, except in the Facilty of Medicine, where a six-year-course is for the M.D. degree. required. One foreign language ta taght in all faculties, the choice being left to the students. The Kabul University has Winter holidays (cnd of N.c. Medicine

Lerinnins of March) the College of in Jalalabed cumner holidays' ( 10 Mey $\left.10 i u_{\mathrm{E}_{\bullet}}\right)$

\section{FICULTY OF ISLUIC LiW}

The Faculty of Islamic Law was founded in 1950. Only students with a final examination from the Islamic school isil Hanife in Bagrami have until now been admitted to the Fac 1 ty. Later on admission also will be granted to students from other Islamic schools.

The Faculty is affiliated with il, Anhr: University in Cairo, and the teaching staff is to a large extent Egyptian. During the present academic year two students of the Taculty are in Cairo on scholarships.

The prinicpal teaching lancuage of the Faculty is Lrabic. Teachers with Dari as their mother tongue sometimes, however, teach in this language.

The graduates from this Faculty usually practice a few years at a lawcourt and then finaliy may become judges themselves. 


\section{FLOJLTY OP LETTERS}

The Faculty of Letters was founded in 1945. It offers its students a four-year course.

Durin: the first year the curriculum is common to all students and comprises such subjects as History, Payrohology, Geography, Logic, English and General Economics.

Commencing with the second year the students go to five different departments, viz:

1. Pashto Department - This Department, where the students devote themselves to the study of the national language Pashto, is closely connected with the Pashto iscademy.

\section{Toresan Departunt.}

3. History and Geography Department.

4. Foreign Language Department. - This Department is divided into the German and the French sections. Until now specialization is. possible only in German, whereas French ts teught in practical courses open to all interested students. Later on specialization in French will be possible.

5. Journalism. - In this depertment courses are given in such general subjects as History, Pashto, Dari, Geography, and Inglish; but the stress is laid upon subjects related to journalistic work, viz: Comparative Journalism, News Writing and Practical Jurnalism. In the dopartments of Pashto, Dari, History and Geography, and Journalism the study of one foreign language is compulsory in addition to the other required subjects. Most students take English, but it is also possible to take irabic, Russian, French or German. The English courses are given in the Faculty of Education.

The teaching language in the Faculty is usuaily Dari, but in the Fashto Department the lectures are given in rashto. Most of the graduates become secondary school teachers. 


\section{FLCULTY OF IULW LND POLITICLI SCI LNCES.}

In 1938 an Institute for Law and Political Sciences was foundod. Lntur this Institutu wig transformed into the present Faculty of Law.

During the first two years the exrieulum is comon for all students, but from the third year there is a choice between two departments, that of Ldministration and that of Diplomatic Service. I.. wech department the duration of studies is two years.

The Faculty is affiliated with la. Fa.ulte de Droit at Sorbonne University in Paris, and this year, 1964, four members of the teaching staff are Fren.h.

Soveral of the graduates of the Faculty continue their studies abroad. This year two are in Franes, one in Switzerland and one in England. Mureover one of the professors spends the year at the Sorbonne.

The terching language of the Faculty is Dari, the foreign professors always being interpreted.

The graduates go, according to their different specialization, to the sdministrative or the diplomatic sorvice.

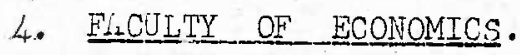

The Faculty of Econcmics is affiliated to the University of Koeln (Federal Republic of Germany). There are two specialities for the third and fourth years:

a) Commercial -conomics

b) National Economics, the first and second years being common. The Diplane at the end of the study corresponds to a Bachelor of Commerce. For admission to post-graduate work, which is mostly carried on in Germany, additional courses must be taken and a preliminary examination must be passed. Teaching language is Dari, but foreign professors teach in German which is translated into Dari. The curriculum covers all subjects pertaining to a modern 4 years' University course in Economics. 


\section{FiCULTY OF SCIENCE.}

The Faculty of Science is affiliated to the University of Bonn (Federal Republic of Germany). There are now four specialties in the second, third and fourth years: Wathematics and Fysics, Chemistry and Biology, Mineralogy and Geology (Mining) and iveteorology. The first year is called MPCB (Mathematics, Physics, Chemistry, Biology) and is sommon for all science furconts. Moreover all future students of the raculties of liedicine and Pharmacy have to pass this ycor at the science Faculty before entering the se Faculties. The teaching language is Dari and courses of foreign professors are translated into Dari by Ifghan kssistants. The Afghan It tomic Energy Commission (ifghanistan being a member of the I.A.E.L (. International stomic Energy Comatssion) has been established in the frame of the Faculty of science, the Chairman of the Commission being thepresent Dean of the Faculty of Science. issistance for different programmes in the field of Nuclear science is given by the I.h.i.... and different bilateral agencies (especially USSR, Hungary and Germany).

\section{6a FLCULTY OF NEDICINE: KiBUI.}

is mentioned above the Faculty of Medinine is the oldest one of Kabul University. In order to raise the standard of the Faculty, a contract was concluded with the Government of France, in accordance with which a number of professors under the direction of the Iyon Medical College assist this Faculty now. The teaching language is Darim courses of foreign professors being translated by assistente into Darl. Technical terms concerning medicine are taken from Latin and israbic. The preparatory year is performed in the MPCB in the Faculty of Scienes and after passing the final examination of this year students may enrol in the first year of the Faculty of Mediaine. Durire five years' study tho following subjocts pertaininge: to the training of a modical practitioner are taught: Histology, linatomy, Biochemistry, Microbiology, Pathology, Dermatology, Internal Medicine, Surgery, Radiology, 
Prevative edicine, Fiar acology, Neurology, Gynaecology, Legal Medicine, Ophthalmology, Pediatrics, O.R.I. (Oto - ihino - Laryngology) and Psychiatry. Lftur orachition ono year of internship in the University Hospital is required, where specialization takes place. UNICEF assists in the supply of Laboratory equipment for Biochemistry, Bacteriology, Pathology and Pharmacology.

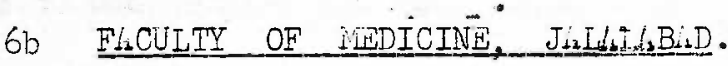

In 1963 a new Faculty $0_{i}$ Medicine was created in jalalabad. It will be the starting point for a University with Pashto as the only teaching language. Bilateral aid from USSR amd Czechoslovakia is available. It Training Hospital for this Faculty is being established. Most of the students come from Pashto speaking areas, especially Nangarhar and Laghman Provinces.

\section{FiLULTI OF PHLPNiLY.}

This Faculty is affiliated to the Faculties of Pharmacy of the French Universities and especially with the Faculty of Pharmacy of Lyon University. Teaching language is Dari, courses of foreign professors are translated into Dari. In 1964 some French professors lectured at the Faculty. Several modern laboratories were established and a vast research programme designed which will cover the following items:
a) medical plants of ifghanistan, collection, classification and utilization
b) control of ifghan and foreign medicaments
c) statistical survey of voluntary and accidental poisoning.

The first year is completed in the science Faculty (IPCB). The second, third and fourth years cover special subjects as Galanic Pharmacy Organic, Biological, snalytical and Pharmaceutical Chemistry, Pharmacology, Merobiology, Lnatomy, Histology, Pharmacodynamics, Cryptogamy, Toxicolosy, Industrial Pharmacy, etc. with the relevant practical work in the laboratories. 


\section{FLGULIY OF EDUCATION.}

The Faculty of Education was founded in 1962 as a part of the Institute of Education. At the beginning of the academic year in 1963 the Faculty became independent of the Institute and has been from that time responsible directly to the University administration.

Durine the first year the curriculum is comnon to all the students with subjects such as English, Pashto, Dari, Social Sciences, Psychology, History, Geography, and Introduction to Education.

From the second year the students specialize either in the Department of English (I) or in the Department of dodministration and supervision.

The teaching language in this Faculty is, except in the English Department, Dari or Pashto, the choice between the languages being dependent on the language which the majority of the class members prefer. In the English Department all courses are taught in inglish expept History of \&fghanistan, Philosophy, Secondary Education, History of Education, Persian Literature and Introduction to Modern Science.

The Teacher'' College, Columbia Univergity Team, U.S.Ls., work closely with the Faculty, and members of this Team are cansultants and instructors in the Faculty.

During the prosent academic year four students are on scholarships at the imerican University in Beirut, Lbbamy and three at Columbia University in IJew York, U.S.h.

Nost of the graduates from the Department English become English teachers in secondary schools. Several possibilities are open to Graduates ff the Department of -dministraion and Supervision. Some of them may practice as teachers in primary or secondary schools to get acquainted with the teaching profession, others may become assistents in school offices or in the offices of provincial directors; some may become teachers of professionsal suljocts in the Dik's,

(1) Prior to 1964 the Department of Einglish belonged to the Faculty of Letters, 
L.fter practicing as teachers or as assistants in oducational offices the graduates of the Department of Ldministration and Supervision are intended to fill positions as school principals or school inspectors or other positions in the school administration. (I)

\section{INSTITUTE OF EDUCLIION.}

The Institute of Education, founded in 1954 as part of the Ministry of Education, was attached to the Univcrity of Kabul in 1955. LIthough not a regular teaching organisetion with its own programme leading to a degree or diplome, the Institute with the assistance and guidance of Members of the tcam from Teachers' College, Columbia University, has been instrumental in providing professional services in the education and training of teachers (see Section IV on Teacher Training). Within the University itself the Institute has been closely associated with the Faculty of Education established in 7962. In 1964 there were $10 \mathrm{Lfghan}$ regular staff members with Frofessorial status in Kabul University. In addition, there were thirty ifghans in the Institute with counterpart status. Institita and TCCU Team members also work closely with the staff members of the Faculty of Education.

(1) Kabul University, Faculty of Education, duplicated brochure, prepared by the Dean of the Faculty and Columbia University Team. 
10 PECULTY OF HOIE ECONOMICS.

This Faculty offers a four years courge for graduates of girls' lycees and is especially concerned with the training of teachers for home economics in firls' schools. Training is also given in home management, child rearing and family management.

The teaching language is Dari. The Curriculum covers all relevant subjects such as Dari, Pashto, English, Food and Nutrition, Textiles, Infectiot's diseases, Cooking, Sewing, First iid, Methods of Teaching, Marriage and family relations, Hone Economics, Phy siology, -rrt, Health, Child Growth and Development, Pyschology, Biology, Chcmistry, Batteriology etc.

\section{WHOUITY OF $\angle$ GRICULTURE.}

This Faculty wes established in 1955. Most of the students are Graduates from the Vocetional igricultureschool, Kabul, Grades X - XII, the LEriculture School and Lycee Baghian (Erades.VIF- XII) and the Institute of Vocational Igriculture (also called Veterinary School) under the Ministry of igriculture.

The Faculty is progressing with the assistance of the USL Wyoming University Team. There are two departments in this Faculty, the Deaprtment of igriculture and that of Veterinary Science. The Teaching language is English, only some subjects in the first year being taught in Dari.

The present curriculum of the Igriculture Department includes the following subjects: English, Basic Mathematics, Botany, Zoology, Microbiology, General, Inorganic and Organic Chemistry, Physics, Genetics, Lgronomy, Intomology, Goils, Field crops, Vegetable production, Fruit froduction, Forestry, Irrigation and drainage, Plant physiology, Plant Patholoøy, Weed control, finimal Production, Dairy elements. 


\section{FLCULTY OF ENGINEERING.}

In the year 1956 a compined Collego for Enginoering and Agriculture was ostablished, but in 1962 the Engineering College was separated and became an independent Faculty.

In October 1.962, the Uihited States Iigency for International Development (USiID) made a contract with the Educational Services Incorporated (ESI) to assist the Kabul Faculty of Engineering, In 1963 a Consortium of US Univorsities sent a group of teachers officially designated as the United States Engineering Team (USET).

There are now two specialties in the thicd and fourth years,viz Civil Engineering and Electro-Mechanical Engineering, the first and second years being common to all students.

Teaching language is English. In order to encourage students from outside Kabul to enter the Faculty and to give them a better chance of success success in the regular course, a short pte-engineering course has been established, which is held from mid-January to mid-March and offers instruction in English, Mathematics and Shop-courses.

iccording to a meeting held in the Faculty of Engineering on November 17th 1964, a short preparatory course in Egglish will be held for graduates of Isteqlal and liedjat and if possible Malalay lycees (French and German as foreigh languiages).

The degree conferred at tho end of the study is a B.Sc.(Eng:). The curriculun of the first and second years includes: English, Engineering practices, Engineering Physics, Engineering Grephics, Engineering Mathemen tics, Physics Laboratory, Statics, Surveyine, Calculue, Dynarics and Physical education.

The CIVIL INGINEERING Section in the third and fourth years includes in the curriculum: Streneth of materials, Blectrical Engineering, Engineering Economic inalysis, idvanced Mathematics, Thermodynamics, Strength of Materials and E'lectrical Ensineering Laboratory, Hydrology, Structural inalysis, Surveying and Maping, Geology, Dynamics, Fluid Vechaniss, Structural Design, Soil Mechanics, terials of Construction, City Planninz, Soil Mechanics Laboratory, Highways, Hydraulics, Irrigation, Sanitary Engineering with Lab.. Hydraulics Lab and Irrigation Lab. 
The Electro-Mechanical Section in the third and fourth years includes in the curriculum: Strength of Materials with Lab, Electrical Engineering with Lab, Engineering Economic Mnalysia, dsdvanced Mathomatics, Thermodynamics, Circuit Inalysis, Dyhamics, Fluid Mechanics, Kinematics, Measuring Techniques, Electronica, Energy conversion, Machine Design, Heat Transfer, Electronics Lab, Mechanical Lab, Electrical Yower and Control, Electrical Machinexy, Industrial Management, Physical Metalurey, Electrical Machines Lab, Matallurgy Lab. 


\section{TRAINING COURSES}

not pertaining to the Ministry of Education.

According to the statistics available in 1962 and 1963 more than 45 courses in which about 800 students were receiving training were run by other Ministries and Institutions. These training centres Care in addition to the courses in operation at the large projects such as Jalabad Canal, Kandahur-Torglundi-Highway and Petroleum Exploration which train about 200 - 400 specialists on-the-job annually in various branches.

In the following a short survey of some of these training fields is given.

\section{Ministry of Agriculture:}

The Ministry of Agriculture trains different specialists in the "Institute of Vocational Agriculture" (known as the "Veterinary School" until last year).

This training centre was established in 1957 and is administered by tho Ministry of Agriculture. Assistance is given by US/AID. The courses are the following:

Courses and number of students and teachers 1964.

Courses VII VIII IX X XI XII TotaI Teaning
staff.

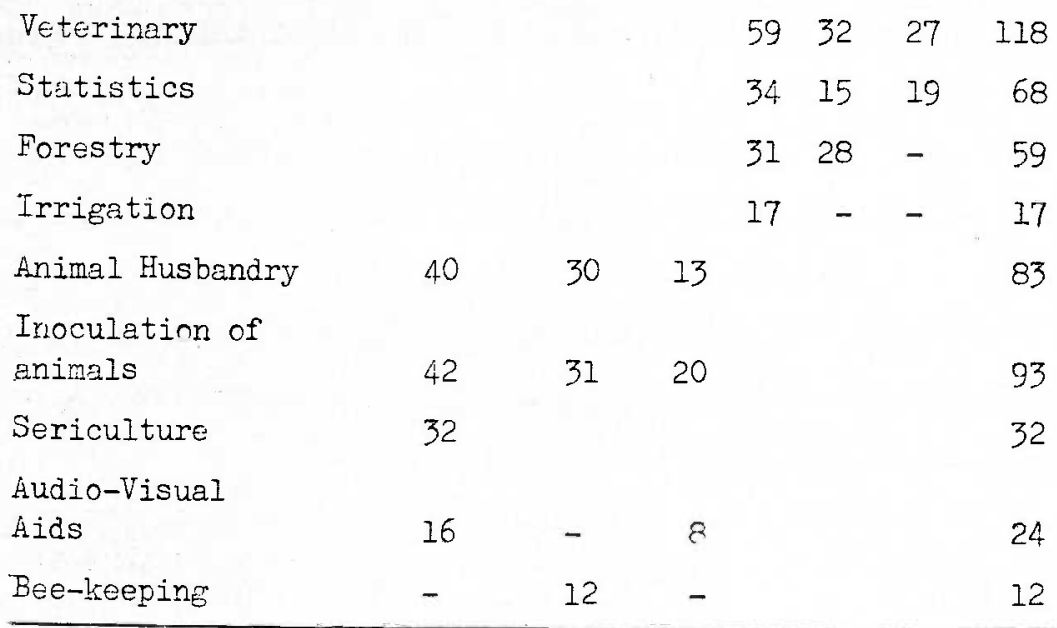




\section{Rural Development.}

According to Survey of Progress 1962 - 64, the Rural Development Iepartment started a comprehensive training programme. A carpentry course tricined ten students in carpentry, in 1962. A new course for the training of Village Workers was launched in 1962 with a complement of 1344 tratnees. A three-weeks' orientation course was held i: 1962 for the henefit of graduate employees and technical personnel of the Rural Develcpment Depertment. Another course was organised for the officers and men of Werdak, Punjab and Chemkan projects in 1963. More than 70 organisers of fundamental education were admitted to the central training centre for a three-months' course of training and instruction in fundamental educstion. A course in handprinting of cloth was organised in 1962 at Charikar. A training course for co-openative workers was successfully completed in 1963. A two-weeks' orientation course for village hecdmen was organised in 1962 with 137 persons and enother with 57 persons in 1963. About 100 persons who attended these courses are already in the field. Four-weeks and three-weeks courses were organised for mural students of the Ishkasham and Logar projects, O.je trainee has been sent abroad under a U.N. programme of technical assistance. The Rural Development Department is sending four to six students every year to the medical school. Upon completion of their trining, they will ke as igned to different projects.

The U.N. Technical Assistance Board relped in the various projects of Rurcil Development. In a six weeks' practical training course Grade XII students of the Kabul D.II.A. are given a course in agriculture, health and handicrafts. An Internetional Labour Organisation expert is conducting a continuous progranme of courses in accountancy and ilanagement techniques for Co-uperetive Organisers employed in the Village Development Schemes. Another I.I.O. expert has been engaged since 1959 in training workers at the Charicar Tannery in moda.n leather tanning processes.

\section{Af ghan Air Authority.}

The facilities established for training in civil aviation by Afghan Air kuthority are the following:-

a) The Kabul Training Centre: This centre takes pupils from all parts of Afghanistan (IX Grade). The number of pupils trained in 1904 was 169 of which 137 were boarders. The courses run were the following:

Hydrology: A three-years' course Grades X-XII. In 1964 the first year of study comprised 26 students. The instructors were Russian experts, instruction wis given in Russian with translation into Dari. 
Meteorolngy: A three-years course, Grades X -XII, in the first year of study in 1964 there were 28 students. Instruction in Russian with translation into Dari.

Radio Maintenance: Course with two classes in 1964, Grades X - XII. 10 students in the first study year and 18 students in the second year. These courses are given in English hy International Civil Aviation Organisation experts with translation into Iari.

Communicstion: I three-years course with 2 classes in 1964.

$\begin{array}{llll}\text { Ist year } & \ldots & \ldots & 11 \text { students } \\ \text { 2nd year } & \ldots & \ldots & 11 \text { students }\end{array}$

These courses are given in English y I.C.A.O. experts with translation into Dari.

Machine-shop Course: This is a six-months' course; pupils come from Grade IX of Mechanical Schools Khost and Kandahar. This course trains specialists for maintenance of airport equipment. In 196410 students were enrolled. Instruction in Russian with translation into Dari (I.C.A.0. experts).

Fire-fighting Course: is six-months course, pupils being taken from Grade VI, 21 stucents in 1964. Instruction in Russian with translation into Dari (I.C.A.O. experts).

Advanced Fire-Fighting Course: On-the-job training for one year. Trainees must rave completed the three-years training in the basic firefighting course. They are destined for service in the airports of Kandahar and Khost. They are now working at the airports in Kandahar and Khost.

Automobile Repairing Course: A three-year on-the-job training for Air huthority in the Jangalak Factory in Kabul. Grades X - XII. 14 pupils take part in this training in 1964 (I.C.A.O. experts).

Radio-maintenance course: 7 students who will go later tc U.S.A. for further training.

(b) The Kandahar Training Centre.

Electronics equipment maintenance course: A two-year course, students are graduates of the Afghan Institute of Technology, Grade XII (Civil Aviation section). Instruction is given hy American experts in English. In 1964 there were 10 students in the second year of study. The graduates of these courses will be distributed to all Afghan airports. 
Mechanical Maintenance Course: A two-year course, with students from the f rrmer Mechanical Section of the Yandahar training centre. They will be trained in the maintenance and service of diesel engines and generators in the airports. In 1964 there were 18 students in the second study year The plane maintenance personnel is trained in the Civil Aviation Section - f the lifghen Institute of Technology. Pilots are als mostly graduates of the i.I.T. (ifghan Institute of Technolcgy) who take a pre-training course at the Eind:insr airport and then ame sent to the U.S.A. as participants on a P.A.A. (Pan limerican Airways) contract.

4. Institute of Public Health.

This Institute organises the fcllowing training courses: Course for Sanitarians: A course which trains sanitarians in a two-year course, corresponding crades XI and XII. The enrolment was the follwwing:

$\begin{array}{lcllccc} & 1959 & 1960 & 1961 & 1962 & 1963 & 1964 \\ \text { Grade XI } & 15 & 20 & 25 & 25 & 28 & 29 \\ \text { Grade XII } & 16 & 15 & 25 & 23 & 25 & 25\end{array}$

One gjrl was trained in Grade XI, all other trainees being boys. The graduates were the following:

$\begin{array}{ccc}1961 & 1962 & 1963 \\ 16 & 20 & 25\end{array}$

On similar lines the following courses are of ered:

Course for Comounders: $\quad 2 \frac{1}{2}$ years-course, graduation corresponds to grade XII. Course for Labor tcry kssistants: $2 \frac{1}{2}$ years-course, graduates equivalent to grade IX.

Course for X-Rey Technicians: $\quad 2 \frac{1}{2}$ years course, graduation equivalent to Grade XII.

Course for fissistant-antists: a four years course, graduation equivalent to Grade XIII.

\section{School of TCpography.}

This schuol is annexed to the Cartographic Institute. It is a krariing school, pupils coming from all parts of the country. The entrants must gradurtes of grade IX (middle schosl).

In 1964 the enrolment was the following:

$$
\begin{aligned}
& \text { Grade X ... ... } 17 \text { studer.ts. } \\
& \text { Grade XI ... ...23 students. } \\
& \text { Grade XII ... .. } 32 \text { students. }
\end{aligned}
$$

The number of gradustes in 1962 was 27 and in 1963.21. A1:. graduates went to the Cartographic Institute. The teachers come from the Cartographic Institute, some for general suljects from the Ministry of Education. 
The curriculum covers the following surjects:

Surveying, Geometry, Algebra, Technical Drawing; History, Geography, Physics, English, Military Training, GeolcEy, Pashto, Triangulation, Phctograminetry, Gecdesy, Astrnnomy, Frojecting methods and Printing. Scholarships fur the graduates are given U.S.A., U.S.S.R. and Czechoslovakia.

\section{Teleconmurication.}

Mairtenance and tperation personnel have been trained during a two-months training course at the existing provisional centre, with the help of I.T.U. (International Telecommication Union). The establishment of a Telecommunication Training Centre has been projected with the aid of U.N. Special Fund. It will be available for the training of higher letel technicians for superrisory duties in a two-year training course, as well as for the training of technicians for the maintenance of telecommunication equipment, systems and circuits, and for refresher courses. The U.N. Special Fund will contribute the necessary training equipment and an international training team. The govermment will contribute the ground and the hildings. Alsc a research centre is included in the project.

\section{Ministry of Finance.}

Within the framewcrl of the Ministry of Finance, different training courses fer employees of the central departments of this Ministry, from other Ministries, from provincial gcvernments and from governmental enterprises are held.

In the following a short survey of these courses is given:

1964 training couraes in the Royal Ministry of Finance:

Number of Trainees 100

\section{6.} 12

60
Ins tructors Afghan.

Budgeting for exployees of Ministries and governuental enterprises

Income tax auditory procedures English language ccurse

There were alsc accounting, supply management, budgeting courses and English courses in 1960, 1961, 1962 and 196:.

\section{Ministry of Mines and Industries.}

The International Iabour Organisution has two projects in operation in this Ministry:

1. Labour Adninistration. An expert in labour administration has conducted training courses in all aspects of personnel menagement and industrial relations for senior cfficials of the Department of Labour and the Personnel Directors of majcr industrial undertukings and developmunt projects. The I.I.0. expert will give further training in these subjects and courses have als heen prepared in labour department administration and labour inspection. In addition the training of potential workers' association officials will be 
2. Clerical Training. A Clerical Training expert has started a class to meet the need frr Roman Script tipists. This Expert will also conduct courses in office procedure for the Ministry's staff.

2. Military Schouls.

Provision for the training of officers and technicians for the army is organised y the Ministry of Defence.

10. Police Academy.

The Academy of Police takes graduates from Lycees to train them in a three-year course for Commissioners. Most of the entrants came in 1964 from the Kahul Lycees but ales some from the provincial schools In 1964 54 students graduated. In attached courses 50 middle level officials and. al patrolien have been graduated.

11. Training in Industries.

Irilling School at Saripul, Mazar-i-Sharif. The Department of Minerals' Survey $y_{i l}^{3}$ in the Royal Ministry of Mines and Industries has established a Irilling School in Saripul in 1961. This school can take about 200 students a year, who have passed grade VI. They are first trained fur a year in the school and then join drilling oparations (on-the-jo training). Trere is training for drillers, de rickmen, diesel-mechanics etc.

On-the-job training is also given in different industries and cunstruction projects, according to Training of Skilled Workers in Afghanistan; Ministry of Planning, Manpower Directirate.

Authority

Jaigalak Factory

Electric Co., Kabul

Textile Co., Jabalseraj

Cement Factory,

Jabalseraj

Textile, Gulbahar Factory

Woolen Faciory, Kabul

Jalabad Canal Project

\section{Training fur Numher to be trained 196.4}

Mor Mecharics, Turners Fitters, Supervisors 160

Electricians 30

Electricians 30

Electricians 20 50 20 Weavers and Spinners 30

Tract.cr-drivers Turbine attendants Shcvel dredgers Electricians Canal masons Fitters Machinists Radi: 'perators Cumpressor mechanics 
Autherity

Tunnel Project

Salang II

Zindabanan Workshop

ivinistry of Communications

Helmand Villey Authority

Ministry of Agriculture
Training for

Number to be trained 1964

Machine repair Mechanics

Constructicn workers

Miotor mechanics

Mechanics

Fitters

Mntor mechanics

Telecummunications personnel

Telegraph and $D$ ist personnel

Line Inspector

Channel System Workers

Postal wurkers

Carpenters

Carpet weaters

Irrigation technicians

Carpenters

Machinists

Plant protection workers 3

15
12

99 20 50 40 20 20 20

10 10

10 15 40

3.

5

(1)

.

40

20

0

25 


\section{FIDESTiLL REPUBLIC OF GLRMENY.}

(i) Fersonnel and equipment for:

(a) Mechanical Schools in Kabul, Kandahar, Whost. Craff Ehovl, Kab

(h) Technical Teahers' Training College, Kabul.

(c) Institute of Industrial Management, Kabul.

(ii) Iniversity of $K_{a}$ bul

(a) Faculty of Science (Bcril University)

(b) Faculty of Economics (Culogne University)

(iii) Volunteer Service Scheme for teachers.

\section{$\underline{\text { FRALICE }}$}

(i) Teachers and laboratory equipment for Malalai and Isteqlal Lycees.

(ii) University of Kabul - affiliations with Universities if Lyons in Medicine and Pharmacy and Faculty of Law of Paris.

Equipment for laburatories in Medicine and Pharmacy and technical

books and pullications for librsies of the three faculties.

\section{INIITED ARAB PEPUBLIC.}

(i) Teachers in Acricultural Schools in Baghlan and Kabul, and in the Islamic Scheols.

(ii) Uilivarsity of Kabul - professors in the Faculti of Theology. UNITED KINGDOM.

(i) Teachers of English under the British Council.

(ii) Volunteer Service Scheme for teschers.

U.S....

Under the Education Division of US/AID assistance is rendered in the form of personnel, eçuipment and buildings. Major projects include:-

(a) Afghin Institute of Technclogy.

(h) Tem of dvisers from Toachers' College, Columbia University, associated with:-

(i) the Institute of Eduction,

(ii) the Faculty of Education,

(iii)English Lengrage Institute of Frculty of Education,

(iv) Teacher Educetion including D.M.A.'s, Emergency Teacher

Training scheme, winter and summer in-service courses.

(v) Comanity School develnment.

(c) Faculty of Agriculture and Agricultural Education - (Wyoming University).

(d) Faculty of Engineering - U.S.E.T.

(e) Peace Corps. 
U.S.S.R.

Technical Education

(a) Technicums (3)

(b) Polytechnic

(c) Sports School.

University - Faculty of Medicine. Alalabar

CZECHOSLOVIIIA.

Visitine professurs in Faculty of Medicine. Tha labed BULGRIA.

Fersonnel in the Construction Bureau in Ministry of Education. AUSTRiA: SCHOOL OF MUSIC FELIOUISHIPS AND SCHOLARSHIPS.

Iducational aid in the form of fellowships and scholarships is made available by many nations. In 1964, over six hundred were provided in different countries, as follows:-

\section{Country}

Belgivil

Bulgaria

Czechos lovakia

Federal Republic of

Germany

France

India

Iran

Italy

Japan

Lebinon (American University of Beirut)
Number

Phillipines

Poland

Switzerland

Thailand

Turkey

U.A.R.

United Kingdom

U.S.A.

U.S.S.R.

Yugoslavia

3

30

I

4

1

62

23

110

114
3

43

In addition 20 Fellowships were provided by the Ministry of Education, 16 by UNiSCO and 6 under the Colombo Plan (Australia).

Short term visits to many countries are arranged from time to time. 